COURSE 3

\title{
FIELD THEORY FOR TRAPPED ATOMIC GASES
}

\author{
H.T.C. STOOF \\ Institute for Theoretical Physics, \\ University of Utrecht. \\ Princetonplein 5 , \\ 3584 CC Utrecht, \\ The Netherlands
}

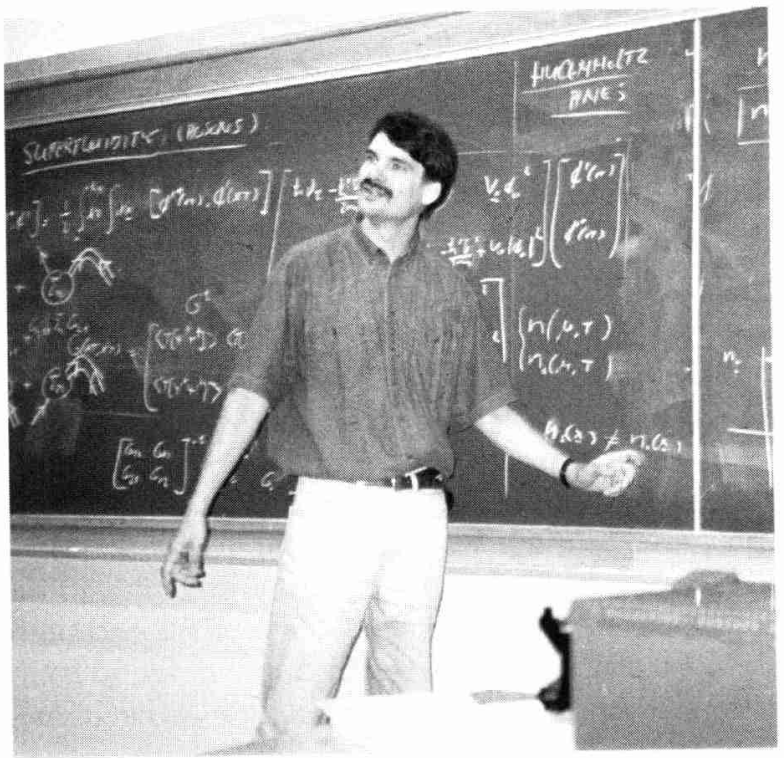




\section{Contents}

1 Introduction $\quad 221$

2 Equilibrium field theory 223

2.1 Second quantization . . . . . . . . . . . . . . . . . . 223

2.2 Grassmann variables and coherent states . . . . . . . . . . 227

2.3 Functional integrals . . . . . . . . . . . . . . . . . . 231

2.4 Ideal quantum gases . . . . . . . . . . . . . . . . . . . . 234

2.5 Interactions and Feynmann diagrams . . . . . . . . . . . . . 240

2.6 Hartree Fock theory for an atomic Fermi gas . . . . . . . . . . . . 245

2.7 Landan theory of phase transitions . . . . . . . . . . . . . . . 249

2.8 Superfluidity and superconductivity . . . . . . . . . 252

3 Nonequilibrium field theory 266

3.1 Macroscopic quantum tunneling of a condensate . . . . . . . . 266

3.2 Phase diffusion . . . . . . . . . . . . . . . . . . . 272

3.3 Quantum kinetic theory . . . . . . . . . . . . . . . . . . . . . . . . . . . . . . . . . . . .

3.4 Condensate formation . . . . . . . . . . . . . . . 295

3.5 Collective modes . . . . . . . . . . . . . . 307

4 Outlook 311 


\title{
FIELD THEORY FOR TRAPPED ATOMIC GASES
}

\author{
H.T.C. Stoof
}

\begin{abstract}
In this course we give a selfcontained introduction to the quantum field theory for trapped atomic gases, using functional methods throughout. We consider both equilibrium and nonequilibrium phenomena. In the equilibrium case, we first derive the appropriate Hartree Fock theory for the properties of the gas in the normal phase. We then turn our attention to the properties of the gas in the superfluid phase, and present a microscopic derivation of the Bogoliubov and Popov theories of Bose-Einstein condensation and the Bardeen-Cooper-Schrieffer theory of superconductivity. The former are applicable to trapped bosonic gases such as rubidium, lithium, sodium and hydrogen, and the latter in particular to the fermionic isotope of atomic lithium. In the nonequilibrium case, we discuss various topics for which a fieldtheoretical approach is especially suited, because they involve physics that is not contained in the Gross-Pitaevskii equation. Examples are quantum kinetic theory, the growth and collapse of a Bose condensate, the phase dynamics of bosonic and fermionic superfluids, and the collisionless collective modes of a Bose gas below the critical temporature.
\end{abstract}

\section{Introduction}

An important trend in the condensed matter physics of the last two decades, has been the use of advanced field-theoretical methods to discuss various subtle and fundamental propertics of interacting many-particle systems at low temperatures. There are several reasons for this trend. The first reason is of course, that a traditional topic in statistical and condensed matter physics is the study of phase transitions and critical phenomena, for which the universal properties are independent of the microscopic details of the system and can therefore be determined by a field theory describing only the large-scale properties of the system of interest. Since the latter are usually solely determined by symmetry considerations, this has led to the important concept of spontaneous symmetry breaking, which has turned out to be not

(C) EDP Sciences, Springer-Verlag 2001 
only highly successful in condensed matter physics, but also in high-energy physics and in particular in the Standard Model of elementary particles [1].

A second reason is that soon after the development of the renormalization group methods for critical phenomena [2], it was realized that the same methods can in fact be used to describe the large-scale properties of manyparticle systems at any temperature and not only near the critical one. Moreover, application of the renormalization group ideas does not only lead to an understanding of the static behaviour but also of the dynamical properties [3]. As a result quantum field theorics can be used to conveniently determine the dynamies of many-particle systems close to equilibrium, i.e.. for example the linear hydrodynamical equations of motion. In addition, it can even be used in highly nonequilibrium situations where in general also nonlinearities play an important role. This feature, that also the dynamics of the system can be captured by a quantum ficld theory, has for instanco led in recent years to the study of so-called quantum phase transitions [4].

Finally, the importance of field-theoretical methods in condensed matter physics is associated with the observation that also the effects of imperfections, i.e., disorder, can be treated in this way [5]. Apart from the technological importance of disorder, for example for superconducting magnets, disorder leads also to fundamentally new physics such as the phenomenon of localization [6]. In quantum Hall systems, a combination of disorder and interaction effects give rise to the realization of various peculiar quantum fluids with fractionally charged excitations [7]. The application of field theories has proven to be highly successful in this case and has led to a theory of the quantum Hall effect in terms of edge states that form a chiral Luttinger liquid [8]. In mesoscopic physics, the study of disorder in small electronic structures has resulted in the so-called random matrix theory [9], which has also been of much use in the study of the quantization of classically chaotic systems.

In this course we aim to show that quantum field theory is also very convenient for obtaining a detailed understanding of the equilibrium and nonequilibrium properties of trapped atomic gases. After the first observations of Bose-Einstein condensation in 1995 [10 12], degenerate atomic gases have received again a great deal of attention and are presently the main subject of study of a large number of experimental and theoretical groups around the world. The reason for all this excitement is, first of all, that Bose-Einstein condensation has never before been observed experimentally in a clear-cut manner, even though this phenomenon was already predicted by Einstein in 1925 [13]. Second, it is of fundamental interest because it is the only phase transition that occurs also in the absense of interactions and is, therefore, the textbook example for the use of statistical-physics methods. Finally, the goal of achieving Bose-Einstein condensation turned out to be much more difficult than anticipated at first. Just before the 
breakthrough in 1995, it had even acquired the nature of a "quest for the holy grail", since the pioneering experiments were already performed in $1980[14]$.

From a theoretical point of view, a quantitative understanding of the experiments with cold atomic gases requires that we take into account the following two effects. First, the gas is magnetically trapped in an, usually axially symmetric, harmonic oscillator potential. This is necessary because, in order to obtain the required record low temperatures of $1-100 \mathrm{nK}$, the gas camnot be allowed to have any contact with material walls. Second, the atoms of the gas interact with each other, which in general dramatically affects the behaviour of the condensed gas, even at the relevant low densities of $10^{12}-10^{1.4} \mathrm{~cm}^{-3}$. An accurate description of these degencrate gases thus requires the solution of a highly inhomogeneous many-body problem. The theoretical challenge posed by these new quantum systems lies therein that the density of the gas is sufficiently small that it should be possible to accurately solve this many-body problem from first principles and to compare the outcome of the theory directly with experiment, i.e., without any adjustable parameters. In our opinion, quantum field theory is the most simple way in which we are able to meet this challenge.

\section{Equilibrium field theory}

We start our development of the quantum field theory of trapped atomic gases by considering first the equilibrium properties of these gases. We consider both Bose and Fermi gases, and the ultimate aim of this section is to arrive, for both cases, at an accurate description of the normal and superfluid phases of the gas. Although mixtures of Bose and Fermi gases are also of current interest, we do not consider them explicitly here, because they can be treated by a straightforward generalization of the theory. In Section 3 we then turn to the noncquilibrium properties, which are perhaps the most interesting and certainly the least understood at present. The reason for organizing the course in this way, is that the development of the equilibrium theory gives us an opportunity to introduce all the necessary tools that are required for a treatment of the more complicated nonequilibrium case. In particular. we present in detail the way in which we can make use of functional methods. To make also a connection with the more familiar operator language, however, we first briefly summarize the outcome of the second quantization formalism.

\subsection{Second quantization}

The atoms of interest to us have internal degrees of freedom due to the electron and nuclear spins. In principle this is very important, because it gives the atom a magnetic moment, which is used to trap the atoms in a 
magnetic field minumum. During this course, however, we restrict ourselves to atomic gases that are a mixture of at most two hyperfine states. Without loss of generality, we can then suppose to have $N$ identical atoms with mass $m$ and effective spin $\mathrm{s}$ in an external potential $V^{\mathrm{ex}}(\mathrm{x})$. As a result. the time-dependent Schrödinger equation we have to solve is

$$
i \hbar \frac{\partial}{\partial t}|\Psi(t)\rangle=\hat{H}|\Psi(t)\rangle .
$$

where the hamiltonian is

$$
\hat{H}=\sum_{i=1}^{N}\left\{\frac{\hat{\mathbf{p}}_{i}^{2}}{2 m}+V^{\mathrm{ex}}\left(\hat{\mathbf{x}}_{i}\right)-\gamma \hat{\mathbf{s}}_{i} \cdot \mathbf{B}^{\mathrm{eff}}\right\}+\frac{1}{2} \sum_{i \neq j=1}^{N} V\left(\hat{\mathbf{x}}_{i}-\hat{\mathbf{x}}_{j}\right) .
$$

$\left[\hat{\mathbf{x}}_{j}, \hat{\mathbf{p}}_{j}\right]_{-}=i \hbar$, and all other commutators of the positions and momenta vanish. The first term in the right-hand side is the sum of the onc-particle hamiltonians, which includes an effective Zeeman interaction that accounts for a possible difference in the hyperfine cnergies. The second term represents the interactions. For simplicity, we have assumed that the interaction $V\left(\hat{\mathbf{x}}_{i}-\hat{\mathbf{x}}_{j}\right)$ is independent of the hyperfine states of the atoms $i$ and $j$. This is in general not justified for realistic atomic gases, but is valid for the specific applications that we have in mind. Moreover, in Section 2.6 we also discuss the general case. Finally, we have also neglected possible three-body forces. This is a result of the fact that we are interested in dilute quantum gases, for which it is highly improbable for three atoms to simultaneously interact with each other.

Without interactions the eigenstates are, of course, given by the states $\left\{\left|\mathbf{n}_{1}, \alpha_{1}\right\rangle_{1} \otimes\left|\mathbf{n}_{2}, \alpha_{2}\right\rangle_{2} \otimes \cdots \otimes\left|\mathbf{n}_{N}, \alpha_{N}\right\rangle_{N}\right\}$, where the specific quantum state for each atom is exactly known. Here $\mathbf{n}=\left(n_{x}, n_{y}, n_{z}\right)$ and the nonnegative integers $n_{x}, n_{y}$, and $n_{z}$ denote the three quantum numbers that are required to specify the one-particle eigenstates in the external potential. The wave functions and energies of these eigenstates are $\chi_{\mathbf{n}}(\mathbf{x}) \equiv\langle\mathbf{x} \mid \mathbf{n}\rangle$ and $\epsilon_{\mathbf{n}}$, respectively, and are found from the time-independent Schrödinger cquation

$$
\left\{-\frac{\hbar^{2} \nabla^{2}}{2 m}+V^{\mathrm{ex}}(\mathrm{x})-\epsilon_{\mathbf{n}}\right\} \chi_{\mathbf{n}}(\mathrm{x})=0 .
$$

In addition, the internal state $|\alpha\rangle$ is a shorthand notation for $\left|s, m_{\mathrm{s}}\right\rangle$. The many-body wave function $|\Psi(t)\rangle$, however, has to be symmetric or antisymmetric under permutations for bosonic or fermionic atoms, respectively. Therefore it is more convenient to use a properly (anti)symmetrized version of the above basis, i.e., the states $\left|\left\{N_{\mathbf{n}, \alpha}\right\}\right\rangle$ with the occupation numbers $N_{\mathbf{n}, \alpha}=0,1,2, \ldots, \infty$ for bosons and $N_{\mathbf{n}, \alpha}=0,1$ for fermions. The Hilbert space of all these states, without the constraint $N=\sum_{\mathbf{n}, \alpha} N_{\mathbf{n}, \alpha}$, is known as the Fock space. 
Clearly, the many-body wave function $|\Psi(t)\rangle$ can be expanded in this basis as

$$
|\Psi(t)\rangle=\sum_{\left\{N_{\mathbf{n}, \alpha}\right\}} \Psi\left(\left\{N_{\mathbf{n}, \alpha}\right\}, t\right)\left|\left\{N_{\mathbf{n}, \alpha}\right\}\right\rangle .
$$

where $\Psi\left(\left\{N_{\mathbf{n}, \alpha}\right\} . t\right)$ is the amplitude for the gas to be in state $\left|\left\{N_{\mathbf{n}, \alpha}\right\}\right\rangle$ at time $t$. In this basis the Schrödinger equation becomes

$$
i \hbar \frac{\partial}{\partial t} \Psi\left(\left\{N_{\mathbf{n} . \alpha}\right\}, t\right)=\sum_{\left\{N_{\mathbf{n}, \alpha}^{\prime}\right\}}\left\langle\left\{N_{\mathbf{n} . \alpha}\right\}|H|\left\{N_{\mathbf{n} . \alpha}^{\prime}\right\}\right) \Psi\left(\left\{N_{\mathbf{n}, \alpha}^{\prime}\right\}, t\right),
$$

which shows that we need the matrix clements of the hamiltonian between different states in the Fock space. To calculate these most easily we introduce so-called annihilation operators $\hat{\psi}_{\mathbf{n} . \alpha}$ by

$$
\hat{\psi}_{\mathbf{n}, \alpha}\left|\ldots, N_{\mathbf{n}, \alpha}, \ldots\right\rangle=\sqrt{N_{\mathbf{n}, \alpha}}\left|\ldots N_{\mathbf{n}, \alpha}-1 \ldots\right\rangle .
$$

from which it follows that the creation operators $\hat{\psi}_{\mathbf{n} . \alpha}^{\dagger}$ obey

$$
\hat{\psi}_{\mathbf{n}, \alpha}^{\dagger}\left|\ldots, N_{\mathbf{n}, \alpha}, \ldots\right\rangle=\sqrt{1 \pm N_{\mathbf{n}, \alpha}}\left|\ldots N_{\mathbf{n}, \alpha}+1, \ldots\right\rangle .
$$

As a result, we see that the operator $\hat{\psi}_{\mathbf{n}, \alpha}^{\dagger} \hat{\psi}_{\mathbf{n} . \alpha}$ counts the number of atoms in the state $|\mathbf{n}, \alpha\rangle$, i.e.,

$$
\hat{\psi}_{\mathbf{n}, \alpha}^{\dagger} \hat{\psi}_{\mathbf{n}, \alpha}\left|\ldots, N_{\mathbf{n}, \alpha}, \ldots\right\rangle=N_{\mathbf{n}, \alpha}\left|\ldots, N_{\mathbf{n}, \alpha}, \ldots\right\rangle .
$$

We have also that $\left[\hat{\psi}_{\mathbf{n}, \alpha}, \hat{\psi}_{\mathbf{n}^{\prime}, \alpha^{\prime}}\right]_{\mp}=\left[\hat{\psi}_{\mathbf{n}, \alpha}^{\dagger}, \hat{\psi}_{\mathbf{n}^{\prime}, \alpha^{\prime}}^{\dagger}\right]_{\mp}=0$ and most importantly that

$$
\left[\hat{\psi}_{\mathbf{n}, \alpha}, \hat{\psi}_{\mathbf{n}^{\prime}, \alpha^{\prime}}^{\dagger}\right]_{\mp}=\delta_{\mathbf{n}, \mathbf{n}^{\prime}} \delta_{\alpha, \alpha^{\prime}}
$$

In equations (2.7) and (2.9) the upper sign refers to bosons and the lower to fermions. This will be true throughout the course.

From these results we can now easily show, first of all, that the basis in the Fock space is given by

$$
\left|\left\{N_{\mathbf{n}, \alpha}\right\}\right\rangle=\prod_{\mathbf{n}, \alpha} \frac{\left(\hat{\psi}_{\mathbf{n}, \alpha}^{\dagger}\right)^{N_{\mathbf{n}, \alpha}}}{\sqrt{N_{\mathbf{n}, \alpha} !}}|0\rangle,
$$

with $|0\rangle$ the vacuum state containing no atoms. Second, the hamiltonian is

$$
\begin{aligned}
\hat{H}= & \sum_{\mathbf{n}, \alpha} \epsilon_{\mathbf{n}, \alpha} \hat{\psi}_{\mathbf{n}, \alpha}^{\dagger} \hat{\psi}_{\mathbf{n}, \alpha} \\
& +\frac{1}{2} \sum_{\alpha, \alpha^{\prime}} \sum_{\mathbf{n}, \mathbf{n}^{\prime}, \mathbf{m}, \mathbf{m}^{\prime}} V_{\mathbf{n}, \mathbf{n}^{\prime} ; \mathbf{m}, \mathbf{m}^{\prime}} \hat{\psi}_{\mathbf{n}, \alpha}^{\dagger} \hat{\psi}_{\mathbf{n}^{\prime}, \alpha^{\prime}}^{\dagger} \hat{\psi}_{\mathbf{m}^{\prime}, \alpha} \hat{\psi}_{\mathbf{m}, \alpha},
\end{aligned}
$$


a)

n, $\alpha$

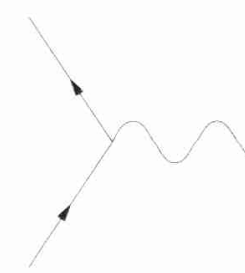

m, $\alpha$ $n^{\prime}, \alpha^{\prime}$

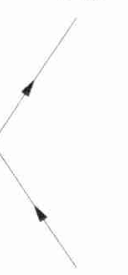

$\mathbf{m}^{\prime}, \alpha^{\prime}$ b)

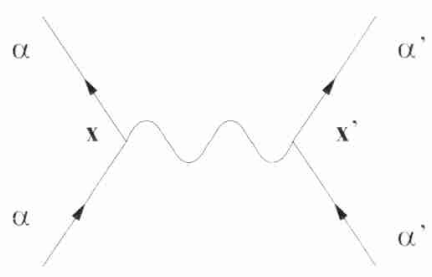

Fig. 1. Diagrammatic representation of the interaction terms in a) equation (2.11) and b) equation (2.13).

with $\epsilon_{\mathbf{n}, \alpha}=\epsilon_{\mathbf{n}}-\gamma m_{\mathrm{s}} B^{\mathrm{eff}} \equiv \epsilon_{\mathbf{n}}+\epsilon_{\alpha}$ the onc-particle energies. Furthormore,

$$
V_{\mathbf{n} \cdot \mathbf{n}^{\prime}: \mathbf{m}, \mathbf{m}^{\prime}}=\int \mathrm{d} \mathbf{x} \int \mathrm{d} \mathbf{x}^{\prime} \chi_{\mathbf{n}}^{*}(\mathbf{x}) \chi_{\mathbf{n}^{\prime}}^{*}\left(\mathrm{x}^{\prime}\right) V\left(\mathbf{x}-\mathrm{x}^{\prime}\right) \chi_{\mathbf{m}}(\mathrm{x}) \chi_{\mathbf{m}^{\prime}}\left(\mathrm{x}^{\prime}\right)
$$

is the amplitude for a collision which scatters two atoms out of the states $|\mathbf{m}, \alpha\rangle$ and $\left|\mathbf{m}^{\prime}, \alpha^{\prime}\right\rangle$ into the states $|\mathbf{n}, \alpha\rangle$ and $\left|\mathbf{n}^{\prime}, \alpha^{\prime}\right\rangle$, as schematically shown in Figure 1(a).

Introducing the field operators $\hat{\psi}_{\alpha}(\mathrm{x})=\sum_{\mathbf{n}} \hat{\psi}_{\mathrm{n}, \alpha} \chi_{\mathbf{n}}(\mathrm{x})$ and $\hat{\psi}_{\alpha}^{\dagger}(\mathrm{x})=$ $\sum_{\mathbf{n}} \hat{\psi}_{\mathbf{n}, \alpha}^{\dagger} \chi_{\mathbf{n}}^{*}(\mathbf{x})$, that annihilate and create atoms in the spin state $|\alpha\rangle$ at position $\mathrm{x}$ respectively, we can rewrite this result into

$$
\begin{aligned}
\hat{H}= & \sum_{\alpha} \int \mathrm{d} \mathbf{x} \hat{\psi}_{\alpha}^{\dagger}(\mathrm{x})\left\{-\frac{\hbar^{2} \nabla^{2}}{2 m}+V^{\mathrm{ex}}(\mathrm{x})+\epsilon_{\alpha}\right\} \hat{\psi}_{\alpha}(\mathrm{x}) \\
& +\frac{1}{2} \sum_{\alpha, \alpha^{\prime}} \int \mathrm{d} \mathrm{x} \int \mathrm{d} \mathrm{x}^{\prime} \hat{\psi}_{\alpha}^{\dagger}(\mathrm{x}) \hat{\psi}_{\alpha^{\prime}}^{\dagger}\left(\mathrm{x}^{\prime}\right) V\left(\mathrm{x}-\mathrm{x}^{\prime}\right) \hat{\psi}_{\alpha^{\prime}}\left(\mathrm{x}^{\prime}\right) \hat{\psi}_{\alpha}(\mathrm{x}) .
\end{aligned}
$$

Note that, due to the (anti)commutation relations of the creation and annihilation operators and the orthogonality of the wave functions $\chi_{\mathbf{n}}(\mathbf{x})$, the field operators obey $\left[\hat{\psi}_{\alpha}(\mathrm{x}), \hat{\psi}_{\alpha^{\prime}}\left(\mathrm{x}^{\prime}\right)\right]_{\mp}=\left[\hat{\psi}_{\alpha}^{\dagger}(\mathrm{x}), \hat{\psi}_{\alpha^{\prime}}^{\dagger}\left(\mathrm{x}^{\prime}\right)\right]_{\mp}=0$ and $\left[\hat{\psi}_{\alpha}(\mathbf{x}), \hat{\psi}_{\alpha^{\prime}}^{\dagger}\left(\mathbf{x}^{\prime}\right)\right]_{\mp}=\delta\left(\mathbf{x}-\mathbf{x}^{\prime}\right) \delta_{\alpha, \alpha^{\prime}}$. Morcover, it is also important for the following to note that the number operator is

$$
\hat{N}=\sum_{\mathbf{n}, \alpha} \hat{\psi}_{\mathbf{n}, \alpha}^{\dagger} \hat{\psi}_{\mathbf{n}, \alpha}=\sum_{\alpha} \int \mathrm{d} \mathbf{x} \hat{\psi}_{\alpha}^{\dagger}(\mathbf{x}) \hat{\psi}_{\alpha}(\mathbf{x})
$$

and, similarly, that the effective total spin operator is

$$
\hat{\mathbf{S}}=\sum_{\mathbf{n}, \alpha, \alpha^{\prime}} \hat{\psi}_{\mathbf{n}, \alpha}^{\dagger}\left\langle\alpha|\hat{\mathbf{s}}| \alpha^{\prime}\right\rangle \hat{\psi}_{\mathbf{n}, \alpha^{\prime}}=\sum_{\alpha, \alpha^{\prime}} \int \mathrm{d} \mathbf{x} \hat{\psi}_{\alpha}^{\dagger}(\mathbf{x})\left\langle\alpha|\hat{\mathbf{s}}| \alpha^{\prime}\right\rangle \hat{\psi}_{\alpha^{\prime}}(\mathbf{x})
$$


The density of atoms in the state $|\alpha\rangle$ is thus simply $\hat{n}_{\alpha}(\mathbf{x})=\hat{\psi}_{\alpha}^{\dagger}(\mathbf{x}) \hat{\psi}_{\alpha}(\mathbf{x})$.

This completes our brief discussion of the second quantization formalism. In principle, we could now proceed to develop an operator formulation of the quantum field theory of interest to us. Since the experimentally most important observables can be expressed as appropriate products of the field operators, as we have just seen. this would essentially amount to the study of the (imaginary) time evolution of the Heisenberg operator $\hat{\psi}(\mathbf{x}, \tau)=\mathrm{e}^{(\hat{H}-\mu \hat{N}) \tau / \hbar} \hat{\psi}_{\alpha}(\mathbf{x}) \mathrm{e}^{-(\hat{H}-\mu \hat{N}) \tau / \hbar}$ at a fixed chemical potential $\mu[15]$. Put differently, the desired quantum field theory would be defined by the Heisenberg equation of motion

$$
\hbar \frac{\partial}{\partial \tau} \hat{\psi}_{\alpha}(\mathbf{x}, \tau)=\left[\hat{H}-\mu \hat{N}, \hat{\psi}_{\alpha}(\mathbf{x}, \tau)\right]_{-},
$$

and we would need to solve this equation in a sufficiently accurate approximation. As mentioned previously, however, we here want to develop Feymman's "path-integral" formulation of the problem, which will turn out to be much more convenient for our purposes. To do so in a manner that is the same for both bosonic and fermionic atomic gases, we first need to introduce some mathematical background.

\subsection{Grassmann variables and coherent states}

We have seen that in the case of fermions, we need to make use of anticommuting creation and annihilation operators. This automatically builds in the Pauli principle in the theory, because it implies that $\left(\hat{\psi_{\mathbf{n}, \alpha}^{\dagger}}\right)^{2}|0\rangle=0$ and thus that the occupation numbers $N_{\mathbf{n}, \alpha}$ are restricted to be either 0 or 1. For reasons that will become clear in a moment, it is in that case also convenient to introduce anticommuting complex numbers or Grassmann variables. The simplest example is to have two such Grassmann variables, say $\phi$ and $\phi^{*}$. The set $\left\{1, \phi, \phi^{*}, \phi^{*} \phi\right\}$, and linear combinations thereof with complex coefficients, form then a so-called Grassmann algebra.

By definition we have $[\phi, \phi]_{+}=\left[\phi, \phi^{*}\right]_{+}=\left[\phi^{*}, \phi^{*}\right]_{+}=0$ and thus in particular $\phi^{2}=\phi^{* 2}=0$. Therefore, the above set is indeed complete. The complex conjugation in this algebra is defined by $(\phi)^{*}=\phi^{*} \cdot\left(\phi^{*}\right)^{*}=\phi$, and $\left(\phi^{*} \phi\right)^{*}=(\phi)^{*}\left(\phi^{*}\right)^{*}=\phi^{*} \phi$. Moreover, we can also define an analytic function on this algebra by

$$
A\left(\phi^{*}, \phi\right)=a_{11}+a_{12} \phi+a_{21} \phi^{*}+a_{22} \phi^{*} \phi .
$$

As a result, it is natural to define also a differentiation by

$$
\frac{\partial}{\partial \phi} A\left(\phi^{*}, \phi\right)=a_{12}-a_{22} \phi^{*} .
$$


To be more precise this is in fact a left differentiation and the minus sign occurs, because we need to permute $\phi^{*}$ and $\phi$ before we can differentiate with respect to $\phi$. So, similarly, we have

$$
\frac{\partial}{\partial \phi^{*}} A\left(\phi^{*} \cdot \phi\right)=a_{21}+a_{22} \phi
$$

and

$$
\frac{\partial^{2}}{\partial \phi^{*} \partial \phi} A\left(\phi^{*}, \phi\right)=-\frac{\partial^{2}}{\partial \phi \partial \phi^{*}} A\left(\phi^{*}, \phi\right)=-a_{22} .
$$

Next we also need integrations over these Grassmann variables. Note that since $\phi^{2}=0$ we have only two possible integrals, namely $\int \mathrm{d} \phi 1$ and $\int \mathrm{d} \phi \phi$. We define these by

$$
\int \mathrm{d} \phi 1=0
$$

and

$$
\int \mathrm{d} \phi \phi=1
$$

This means that integration is equivalent to differentation. The main reason for the above definition is that we want the integration to obey the nusual rules of partial integration. In particular, this implies that

$$
\int \mathrm{d} \phi \frac{\partial F(\phi)}{\partial \phi}=0
$$

for any function $F(\phi)=f_{1}+f_{2} \phi$. It is clear that this condition requires that $\int \mathrm{d} \phi 1=0$. The result of $\int \mathrm{d} \phi \phi$ is then solely a question of normalization. It turns out that we are primarily interested in integrals of the form

$$
\int \mathrm{d} \phi^{*} \mathrm{~d} \phi A\left(\phi^{*}, \phi\right)=\int \mathrm{d} \phi^{*} \mathrm{~d} \phi\left(a_{11}+a_{12} \phi+a_{21} \phi^{*}+a_{22} \phi^{*} \phi\right)=-a_{22}
$$

as we will see in Section 2.3.

Clearly, we can now also consider the Grassmann algebra based on the variables $\phi_{n}$ and $\phi_{n}^{*}$ with $n=1,2 \ldots, \infty$. What we will need in the following are gaussian integrals over these variables. It is not difficult to show with the above definitions that

$$
\int\left(\prod_{n} \mathrm{~d} \phi_{n}^{*} \mathrm{~d} \phi_{n}\right) \exp \left\{-\sum_{n, n^{\prime}} \phi_{n}^{*} A_{n, n^{\prime}} \phi_{n^{\prime}}\right\}=\operatorname{det} A=\mathrm{e}^{\operatorname{Tr}[\ln A]} .
$$


If the variables $\phi_{n}$ and $\phi_{n}^{*}$ were just ordinary complex numbers we would in contrast have the result

$$
\int\left(\prod_{n} \frac{\mathrm{d} \phi_{n}^{*} \mathrm{~d} \phi_{n}}{2 \pi i}\right) \exp \left\{-\sum_{n, n^{\prime}} \phi_{n}^{*} A_{n, n^{\prime}} \phi_{n^{\prime}}\right\}=\frac{1}{\operatorname{det} A}=\mathrm{e}^{-\operatorname{Tr}[\ln A]} .
$$

These last two results will be used many times in the following.

One immediate use of these Grassmann variables is that we can now consider eigenstates of the annihilation operator $\hat{\psi}_{\mathbf{n}, \alpha}$, also when we are dealing with fermions. These eigenstates are called coherent states. Consider the state

$$
\left|\phi_{\mathbf{n}, \alpha}\right\rangle \equiv\left(1-\phi_{\mathbf{n}, \alpha} \hat{\psi}_{\mathbf{n}, \alpha}^{\dagger}\right)|0\rangle=\exp \left\{-\phi_{\mathbf{n}, \alpha} \hat{\psi}_{\mathbf{n}, \alpha}^{\dagger}\right\}|0\rangle
$$

where $\phi_{\mathbf{n}, \alpha}$ is a Grassmann variable that also anticommutes with the creation and ammihilation operators in our Fock space. Clearly we have that

$$
\begin{aligned}
\hat{\psi}_{\mathbf{n}, \alpha}\left|\phi_{\mathbf{n}, \alpha}\right\rangle & =\hat{\psi}_{\mathbf{n}, \alpha}|0\rangle+\phi_{\mathbf{n}, \alpha} \hat{\psi}_{\mathbf{n}, \alpha} \hat{\psi}_{\mathbf{n}, \alpha}^{\dagger}|0\rangle \\
& =\phi_{\mathbf{n}, \alpha}|0\rangle=\phi_{\mathbf{n}, \alpha}\left(1-\phi_{\mathbf{n}, \alpha} \hat{\psi}_{\mathbf{n}, \alpha}^{\dagger}\right)|0\rangle=\phi_{\mathbf{n}, \alpha}\left|\phi_{\mathbf{n}, \alpha}\right\rangle,
\end{aligned}
$$

so $\left|\phi_{\mathbf{n}, \alpha}\right\rangle$ is indeed an eigenstate of $\hat{\psi}_{\mathbf{n}, \alpha}$ with eigenvalue $\phi_{\mathbf{n}, \alpha}$. In general we can now make the states

$$
|\phi\rangle=\exp \left\{-\sum_{\mathbf{n}, \alpha} \phi_{\mathbf{n}, \alpha} \hat{\psi}_{\mathbf{n}, \alpha}^{\dagger}\right\}|0\rangle
$$

that obey $\hat{\psi}_{\mathbf{n}, \alpha}|\phi\rangle=\phi_{\mathbf{n}, \alpha}|\phi\rangle$. Introducing the Grassmann-valued field $\phi_{\alpha}(\mathbf{x})=\sum_{\mathbf{n}} \phi_{\mathbf{n}, \alpha} \chi_{\mathbf{n}}(\mathbf{x})$, the latter two relations can be rewritten as

$$
|\phi\rangle=\exp \left\{-\sum_{\alpha} \int \mathrm{d} \mathbf{x} \phi_{\alpha}(\mathrm{x}) \hat{\psi}_{\alpha}^{\dagger}(\mathrm{x})\right\}|0\rangle
$$

and $\hat{\psi}_{\alpha}(\mathbf{x})|\phi\rangle=\phi_{\alpha}(\mathbf{x})|\phi\rangle$.

It is important to note that these coherent states are not orthonormal. In contrast, we find that

$$
\begin{aligned}
\left\langle\phi \mid \phi^{\prime}\right\rangle & =\prod_{\mathbf{n}, \alpha}\left\langle 0\left|\left(1-\hat{\psi}_{\mathbf{n}, \alpha} \phi_{\mathbf{n}, \alpha}^{*}\right)\left(1-\phi_{\mathbf{n}, \alpha}^{\prime} \hat{\psi}_{\mathbf{n}, \alpha}^{\dagger}\right)\right| 0\right\rangle \\
& =\prod_{\mathbf{n}, \alpha}\left(1+\phi_{\mathbf{n}, \alpha}^{*} \phi_{\mathbf{n}, \alpha}^{\prime}\right)=\exp \left\{\sum_{\mathbf{n}, \alpha} \phi_{\mathbf{n}, \alpha}^{*} \phi_{\mathbf{n}, \alpha}^{\prime}\right\} \\
& =\exp \left\{\sum_{\alpha} \int \mathrm{d} \mathbf{x} \phi_{\alpha}^{*}(\mathbf{x}) \phi_{\alpha}^{\prime}(\mathbf{x})\right\} \equiv \mathrm{e}^{\left(\phi \mid \phi^{\prime}\right)} .
\end{aligned}
$$


Nevertheless they obey a closure relation, as can be seen explicitly from

$$
\begin{aligned}
\int & \left(\prod_{\mathbf{n}, \alpha} \mathrm{d} \phi_{\mathbf{n}, \alpha}^{*} \mathrm{~d} \phi_{\mathbf{n}, \alpha}\right) \exp \left\{-\sum_{\mathbf{n}, \alpha} \phi_{\mathbf{n}, \alpha}^{*} \phi_{\mathbf{n}, \alpha}\right\}|\phi\rangle\langle\phi| \\
= & \prod_{\mathbf{n}, \alpha} \int \mathrm{d} \phi_{\mathbf{n}, \alpha}^{*} \mathrm{~d} \phi_{\mathbf{n}, \alpha}\left(1-\phi_{\mathbf{n}, \alpha}^{*} \phi_{\mathbf{n}, \alpha}\right)\left(1-\phi_{\mathbf{n}, \alpha} \hat{\psi}_{\mathbf{n}, \alpha}^{\dagger}\right)|0\rangle\langle 0|\left(1-\hat{\psi}_{\mathbf{n}, \alpha} \phi_{\mathbf{n}, \alpha}^{*}\right) \\
= & \prod_{\mathbf{n}, \alpha}(|0\rangle\langle 0|+| 1\rangle\langle 1|)=\hat{1} .
\end{aligned}
$$

We write this from now on simply as

$$
\int \mathrm{d}\left[\phi^{*}\right] \mathrm{d}[\phi] \mathrm{e}^{-(\phi \mid \phi)}|\phi\rangle\langle\phi|=\hat{1} .
$$

The interesting observation at this point is that essentially the same formulaes also hold for bosons $[16,17]$. We have only a minus sign difference in

$$
|\phi\rangle=\exp \left\{\sum_{\alpha} \int \mathrm{dx} \phi_{\alpha}(\mathrm{x}) \hat{\psi}_{\alpha}^{\dagger}(\mathrm{x})\right\}|0\rangle .
$$

but then it can still be easily shown that $\hat{\psi}_{\alpha}(\mathrm{x})|\phi\rangle=\phi_{\alpha}(\mathrm{x})|\phi\rangle$. A convenient way to do so is, for instance, by noting that the commutation relation $\left[\hat{\psi}_{\alpha}(\mathbf{x}), \hat{\psi}_{\alpha^{\prime}}^{\dagger}\left(\mathbf{x}^{\prime}\right)\right]_{-}=\delta\left(\mathbf{x}-\mathbf{x}^{\prime}\right) \delta_{\alpha, \alpha^{\prime}}$ implies that $\hat{\psi}_{\alpha}(\mathbf{x})$ acts as $\delta / \delta \hat{\psi}_{\alpha}^{\dagger}(\mathbf{x})$ on these states. Furthermore, the same expressions hold for the overlap $\left\langle\phi \mid \phi^{\prime}\right\rangle$ and the closure relation, if we define the integration measure by $\int \mathrm{d}\left[\phi^{*}\right] \mathrm{d}[\phi] \equiv \int \prod_{\mathbf{n}, \alpha} \mathrm{d} \phi_{\mathbf{n}, \alpha}^{*} \mathrm{~d} \phi_{\mathbf{n}, \alpha} /(2 \pi i)$ in this case.

Summarizing, we have thus found for bosons and fermions that

$$
\begin{gathered}
|\phi\rangle=\exp \left\{ \pm \sum_{\alpha} \int \mathrm{d} \mathbf{x} \phi_{\alpha}(\mathbf{x}) \hat{\psi}_{\alpha}^{\dagger}(\mathbf{x})\right\}|0\rangle, \\
\left\langle\phi \mid \phi^{\prime}\right\rangle=\mathrm{e}^{\left(\phi \mid \phi^{\prime}\right)},
\end{gathered}
$$

and

$$
\int \mathrm{d}\left[\phi^{*}\right] \mathrm{d}[\phi] \mathrm{e}^{-(\phi \mid \phi)}|\phi\rangle\langle\phi|=\hat{1}
$$

The last ingredient we need is that in terms of these coherent states the trace of an operator $\hat{O}$ over the Fock space can be expressed as

$$
\operatorname{Tr}[\hat{O}]=\int \mathrm{d}\left[\phi^{*}\right] \mathrm{d}[\phi] \mathrm{e}^{-(\phi \mid \phi)}\langle \pm \phi|\hat{O}| \phi\rangle .
$$


The minus sign in the fermionic case is easily understood from the fact that we then have $\left\langle\left\{N_{\mathbf{n}, \alpha}\right\} \mid \phi\right\rangle\left\langle\phi \mid\left\{N_{\mathbf{n}, \alpha}\right\}\right\rangle=\left\langle-\phi \mid\left\{N_{\mathbf{n}, \alpha}\right\}\right\rangle\left\langle\left\{N_{\mathbf{n}, \alpha}\right\} \mid \phi\right\rangle$, due to the anticommuting nature of the Grassmamn variables. After this mathematical interlude we can now return to physics and to our goal of arriving at a unified treatment of bosonic and fermionic atomic gases.

\subsection{Functional integrals}

We are interested in determining the equilibrium properties of a trapped atomic gas at some temperature $T$. From statistical physics we know that all these properties can be obtained from the grand-canonical partition function

$$
Z=\operatorname{Tr}\left[\mathrm{e}^{-\beta(\hat{H}-\mu \hat{N})}\right]
$$

where $\beta=1 / k_{B} T$ and $\mu$ the chemical potential. We thus need to evaluate this quantity. As mentioned previously, we want to do so by making use of quantum field theory and, in addition, of Feynman's path-integral approach to quantum mechanics. We thus want to write the partition function as a functional integral over time-dependent fields $\phi_{\alpha}(\mathrm{x}, \tau)$. just like the partition function of a single particle in an external potential can be written as a functional integral over all time-dependent paths $\mathbf{x}(\tau)$. This can indeed be achieved with the cohcrent states that we have introduced in the previous section.

We start with using our formula (2.38) for the trace of an operator,

$$
Z=\int \mathrm{d}\left[\phi^{*}\right] \mathrm{d}[\phi] \mathrm{e}^{-(\phi \mid \phi)}\left\langle \pm \phi\left|\mathrm{e}^{-\beta(H-\mu \hat{N})}\right| \phi\right\rangle,
$$

and observe that we are thus faced with the task of calculating the matrix elements $\left\langle\phi_{M}\left|\mathrm{e}^{-\beta(\hat{H}-\mu \hat{N})}\right| \phi_{0}\right\rangle$ with $\phi_{0}(\mathrm{x})=\phi(\mathrm{x})$ and $\phi_{M}^{*}(\mathrm{x})= \pm \phi^{*}(\mathrm{x})$. This is difficult in general but can be simplified in the following way. We first realize that the operator $\mathrm{e}^{-\beta(\hat{H}-\mu \hat{N})}$ is identical to the quantum mechanical evolution operator $U(t, 0)=\mathrm{e}^{-i(\hat{H}-\mu \hat{N}) t / \hbar}$ evaluated at $t=-i \hbar \beta$. Put differently, we thus want to calculate the matrix elements of the imaginarytime cvolution operator $U(-i \tau, 0)$ for $\tau=\hbar \beta$. To do so, we next split the time interval $[0, \hbar \beta]$ into $M$ pieces, with $\tau_{m}=m \hbar \beta / M$ and $m=0,1, \ldots, M$. So $\Delta \tau=\hbar \beta / M$. The procedure is summarized in Figure 2.

At each intermediate time $\tau_{m}$ we then apply a closure relation of the coherent states. This gives

$$
\begin{aligned}
& \left\langle\phi_{M}\left|\mathrm{e}^{-\beta(\hat{H}-\mu \hat{N})}\right| \phi_{0}\right\rangle \\
& =\int\left(\prod_{m=1}^{M-1} \mathrm{~d}\left[\phi_{m}^{*}\right] \mathrm{d}\left[\phi_{m}\right] \mathrm{e}^{-\left(\phi_{m} \mid \phi_{m}\right)}\right) \prod_{m=1}^{M}\left\langle\phi_{m}\left|\mathrm{e}^{-\Delta \tau(\hat{H}-\mu \hat{N}) / \hbar}\right| \phi_{m-1}\right\rangle .
\end{aligned}
$$




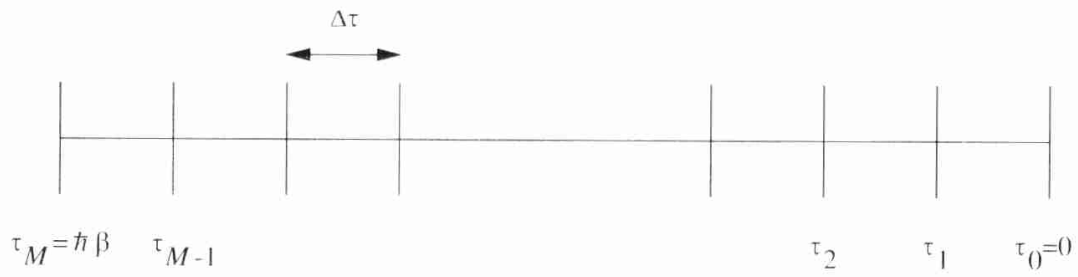

Fig. 2. Illustration of the slicing of the imaginary time interval $[0, \hbar, \beta]$ needed for Feynman's path-integral formulation of the partition function.

Now we can use that in the limit $M \rightarrow \infty$ we only need to know the latter matrix elements up to order $\Delta \tau$, because terms of order $(\Delta \tau)^{2}$ lead to corrections of order $M(\Delta \tau)^{2} \propto 1 / M$, which vanish in that limit. Hence

$$
\begin{aligned}
\left\langle\phi_{m}\left|\mathrm{e}^{-\Delta \tau(\hat{H}-\mu \hat{N}) / \hbar}\right| \phi_{m-1}\right\rangle & \simeq\left\langle\phi_{m}|1-\Delta \tau(\hat{H}-\mu \hat{N}) / \hbar| \phi_{m-1}\right\rangle \\
& \equiv\left\langle\phi_{m} \mid \phi_{m-1}\right\rangle\left(1-\Delta \tau H\left[\phi_{m}^{*}, \phi_{m-1}\right] / \hbar\right),
\end{aligned}
$$

with the grand-canonical hamiltonian functional resulting from equations (2.13) and (2.14) equal to

$$
\begin{aligned}
H\left[\phi^{*}, \phi\right] & =\sum_{\alpha} \int \mathrm{d} \mathbf{x} \phi_{\alpha}^{*}(\mathbf{x})\left\{-\frac{\hbar^{2} \nabla^{2}}{2 m}+V^{\mathrm{ex}}(\mathbf{x})+\epsilon_{\alpha}-\mu\right\} \phi_{\alpha}(\mathbf{x}) \\
& +\frac{1}{2} \sum_{\alpha, \alpha^{\prime}} \int \mathrm{d} \mathbf{x} \int \mathrm{d} \mathbf{x}^{\prime} \phi_{\alpha}^{*}(\mathbf{x}) \phi_{\alpha^{\prime}}^{*}\left(\mathbf{x}^{\prime}\right) V\left(\mathbf{x}-\mathrm{x}^{\prime}\right) \phi_{\alpha^{\prime}}\left(\mathrm{x}^{\prime}\right) \phi_{\alpha}(\mathbf{x}),
\end{aligned}
$$

since $\psi_{\alpha}(\mathbf{x})|\phi\rangle=\phi_{\alpha}(\mathbf{x})|\phi\rangle$ and $\langle\phi| \psi_{\alpha}^{\dagger}(\mathbf{x})=\langle\phi| \phi_{\alpha}^{*}(\mathbf{x})$.

Thus, neglecting terms of order $(\Delta \tau)^{2}$, we can again exponentiate the right-hand side of equation (2.42), which leads in first instance to

$$
\left\langle\phi_{m}\left|\mathrm{e}^{-\Delta \tau(\hat{H}-\mu \hat{N}) / \hbar}\right| \phi_{m-1}\right\rangle=\mathrm{e}^{\left(\phi_{m} \mid \phi_{m-1}\right)-\Delta \tau H\left[\phi_{m}^{*}, \phi_{m-1}\right] / h},
$$

and therefore for the desired matrix element of the imaginary-time evolution operator to

$$
\begin{aligned}
\left\langle\phi_{M}\left|\mathrm{e}^{-\beta(\hat{H}-\mu \hat{N})}\right| \phi_{0}\right\rangle= & \int\left(\prod_{m=1}^{M-1} \mathrm{~d}\left[\phi_{m}^{*}\right] \mathrm{d}\left[\phi_{m}\right] \mathrm{e}^{-\left(\phi_{m} \mid \phi_{m}\right)}\right) \\
& \times \exp \left\{\sum_{m=1}^{M}\left(\left(\phi_{m} \mid \phi_{m-1}\right)-\Delta \tau H\left[\phi_{m}^{*}, \phi_{m-1}\right] / \hbar\right)\right\} .
\end{aligned}
$$


This can then be manipulated into the suggestive form

$$
\begin{aligned}
& \left\langle\phi_{M}\left|\mathrm{e}^{-\beta(H-\mu N)}\right| \phi_{0}\right\rangle=\mathrm{e}^{\left(\phi_{M} \mid \phi_{M}\right)} \int\left(\prod_{m=1}^{M-1} \mathrm{~d}\left[\phi_{m}^{*}\right] \mathrm{d}\left[\phi_{m}\right]\right) \\
& \quad \times \exp \left\{-\frac{1}{\hbar} \sum_{m=1}^{M} \Delta \tau\left(\hbar \frac{\left(\phi_{m} \mid \phi_{m}\right)-\left(\phi_{m} \mid \phi_{m-1}\right)}{\Delta \tau}+H\left[\phi_{m}^{*}, \phi_{m-1}\right]\right)\right\} .
\end{aligned}
$$

Taking now the continuum limit $M \rightarrow \infty$ and putting $\phi_{m} \equiv \phi\left(\tau_{m}\right)$, we find that

$$
\begin{aligned}
& \left\langle\phi_{M}\left|\mathrm{c}^{-\beta(H-\mu N)}\right| \phi_{0}\right\rangle= \\
& \qquad \mathrm{e}^{(\phi(\hbar \beta) \mid \phi(\hbar \beta))} \int_{\phi(0)=\phi_{0}}^{\phi^{*}(\hbar \beta)=\phi_{M}^{*}} \mathrm{~d}\left[\phi^{*}\right] \mathrm{d}[\phi] \mathrm{e}^{-S\left[\phi^{*}, \phi\right] / \hbar},
\end{aligned}
$$

where the so-called Euclidean action is

$$
\begin{aligned}
& S\left[\phi^{*}, \phi\right]= \\
& \int_{0}^{h \beta} \mathrm{d} \tau\left\{\sum_{\alpha} \int \mathrm{dx} \phi_{\alpha}^{*}(\mathrm{x}, \tau) \hbar \frac{\partial}{\partial \tau} \phi_{\alpha}(\mathrm{x}, \tau)+H\left[\phi^{*}(\tau), \phi(\tau)\right]\right\} .
\end{aligned}
$$

This is essentially the desired functional integral over the complex fields $\phi_{\alpha}(\mathbf{x}, \tau)$ with the boundary conditions $\phi_{\alpha}(\mathbf{x}, 0)=\phi_{0 ; \alpha}(\mathbf{x})$ and $\phi_{\alpha}^{*}(\mathbf{x}, \hbar \beta)=$ $\phi_{M ; \alpha}^{*}(\mathrm{x})$. It is precisely the field theory analogue of the Feymman path integral. To obtain the partition function we only need to put $\phi_{0}(\mathrm{x})$ equal to $\pm \phi_{M}(\mathrm{x})$ and perform a last integration over $\phi_{M}(\mathrm{x})$ and $\phi_{M}^{*}(\mathrm{x})$. It then finally becomes

$$
Z=\int \mathrm{d}\left[\phi^{*}\right] \mathrm{d}[\phi] \mathrm{e}^{-S\left[\phi^{*} \cdot \phi\right] / \hbar}
$$

with the boundary conditions $\phi_{\alpha}(\mathbf{x}, \hbar \beta)= \pm \phi_{\alpha}(\mathbf{x}, 0)$, i.e., the fields are periodic in $[0, \hbar \beta]$ for bosons and antiperiodic for fermions. Note that in equation (2.47) we have used the same notation for the integration measure as in equation (2.49), although there is in principle one more integration in the expression for the partition function. The difference is in the continuum limit accounted for in the boundary conditions, which are in practice usually left implicite. Having arrived at an exact identity between the partition function and a functional integral, we are now going to familiarize ourselves with this identity, and with how to perform functional integrals in general, by considering the ideal quantum gases. 


\subsection{Ideal quantum gases}

Since the partition functions $Z_{0}$ of the ideal quantum gases are known exactly, they are ideal test cases for our field-theoretical methods. Moreover, a thorough knowledge of the ideal quantum gases is also an important first step in understanding experiments with trapped atomic gases, because the effects of the interatomic interaction can essentially be included perturbatively. How this perturbation theory is performed is discussed in detail in Section 2.5 , but before we can do that we need to understand the noninteracting gases first. In that case, we have

$$
\begin{aligned}
S_{0}\left[\phi^{*}, \phi\right]= & \sum_{\alpha} \int_{0}^{\hbar \beta} \mathrm{d} \tau \int \mathrm{d} \mathbf{x} \\
& \times \phi_{\alpha}^{*}(\mathbf{x}, \tau)\left\{\hbar \frac{\partial}{\partial \tau}-\frac{\hbar^{2} \nabla^{2}}{2 m}+V^{\mathrm{ex}}(\mathbf{x})+\epsilon_{\alpha}-\mu\right\} \phi_{\alpha}(\mathbf{x}, \tau)
\end{aligned}
$$

and the partition function is a Gaussian integral, which explains why we were interested in Gaussian integrals in Section 2.2. It will be illustrative to evaluate this partition function in three different ways.

\subsubsection{Semiclassical method}

In the first way, we perform the evaluation of the trace involved in the definition of the partition function at the end of the calculation, and start by considering the matrix element $\left\langle \pm \phi\left|\mathrm{e}^{-\beta(\hat{H}-\mu \hat{N})}\right| \phi\right\rangle$ as the functional integral

$$
\begin{aligned}
\int_{\phi(0)=\phi}^{\phi^{*}(h \beta)= \pm \phi^{*}}\left(\prod_{\mathbf{n}, \alpha} \mathrm{d}\left[\phi_{\mathbf{n}, \alpha}^{*}\right] \mathrm{d}\left[\phi_{\mathbf{n}, \alpha}\right]\right) \exp \left\{\sum_{\mathbf{n}, \alpha} \phi_{\mathbf{n}, \alpha}^{*}(h \beta) \phi_{\mathbf{n}, \alpha}(h \beta)\right\} \\
\times \exp \left\{-\frac{1}{\hbar} \int_{0}^{\hbar \beta} \mathrm{d} \tau \sum_{\mathbf{n}, \alpha} \phi_{\mathbf{n}, \alpha}^{*}(\tau)\left(h \frac{\partial}{\partial \tau}+\epsilon_{\mathbf{n}, \alpha}-\mu\right) \phi_{\mathbf{n}, \alpha}(\tau)\right\},
\end{aligned}
$$

which is the product for each $\mathbf{n}$ and $\alpha$ of the path integral

$$
\int \mathrm{d}\left[\phi^{*}\right] \mathrm{d}[\phi] \exp \left\{\phi^{*}(\hbar \beta) \phi(\hbar \beta)-\frac{1}{\hbar} \int_{0}^{\hbar \beta} \mathrm{d} \tau \phi^{*}(\tau)\left(\hbar \frac{\partial}{\partial \tau}+\epsilon-\mu\right) \phi(\tau)\right\}
$$

with the boundary conditions $\phi(0)=\phi$ and $\phi(\hbar \beta)= \pm \phi^{*}$. It clearly corresponds to the matrix element $\left\langle \pm \phi\left|\mathrm{e}^{-\beta(\epsilon-\mu) \hat{\psi}^{+} \hat{\psi}}\right| \phi\right\rangle$ for one particular value of $\mathbf{n}$ and $\alpha$. We calculate this matrix element by performing a shift in the integration variables, i.e., $\phi(\tau)=\phi_{\mathrm{cl}}(\tau)+\xi(\tau)$ and $\phi^{*}(\tau)=\phi_{\mathrm{cl}}^{*}(\tau)+\xi^{*}(\tau)$. where $\phi_{\mathrm{cl}}(\tau)$ obeys the "classical" equations of motion

$$
\left.\frac{\delta S_{0}\left[\phi^{*}, \phi\right]}{\delta \phi^{*}(\tau)}\right|_{\phi=\phi_{c 1}}=\left(\hbar \frac{\partial}{\partial \tau}+\epsilon-\mu\right) \phi_{\mathrm{cl}}(\tau)=0
$$


and similarly for $\phi_{\mathrm{cl}}^{*}(\tau)$. The solutions with the correct boundary solutions are $\phi_{\mathrm{cl}}(\tau)=\phi \mathrm{e}^{-(\epsilon-\mu) \tau / \hbar}$ and $\phi_{\mathrm{cl}}^{*}(\tau)= \pm \phi^{*} \mathrm{e}^{(\epsilon-\mu)(\tau-\hbar \beta) / \hbar}$, leading to the path integral

$$
\begin{aligned}
& \exp \left\{ \pm \mathrm{e}^{-\beta(\epsilon-\mu)} \phi^{*} \phi\right\} \\
& \qquad \times \int \mathrm{d}\left[\xi^{*}\right] \mathrm{d}[\xi] \exp \left\{-\frac{1}{\hbar} \int_{0}^{\hbar \beta} \mathrm{d} \tau \xi^{*}(\tau)\left(\hbar \frac{\partial}{\partial \tau}+\epsilon-\mu\right) \xi(\tau)\right\}
\end{aligned}
$$

with the boundary conditions $\xi^{*}(\hbar \beta)=\xi(0)=0$. This means that the last path integral is just equal to $\left\langle 0\left|\mathrm{e}^{-\beta(\epsilon-\mu) \hat{\psi}^{\dagger} \psi}\right| 0\right\rangle=1$ and that the desired result is just the prefactor.

Substituting this for each value of $\mathbf{n}$ and $\alpha$, we apparently have

$$
\left\langle \pm \phi\left|\mathrm{e}^{-\beta(\hat{H}-\mu \hat{N})}\right| \phi\right\rangle=\exp \left\{ \pm \sum_{\mathbf{n}, \alpha} \mathrm{e}^{-\beta\left(\epsilon_{\mathbf{n}, \alpha}-\mu\right)} \phi_{\mathbf{n}, \alpha}^{*} \phi_{\mathbf{n}, \alpha}\right\}
$$

and the partition function becomes the product

$$
\begin{aligned}
Z_{0} & =\prod_{\mathbf{n}, \alpha} \int \frac{\mathrm{d} \phi_{\mathbf{k}, \alpha}^{*} \mathrm{~d} \phi_{\mathbf{k}, \alpha}}{(2 \pi i)^{(1 \pm 1) / 2}} \exp \left\{-\left(1 \mp \mathrm{e}^{-\beta\left(\epsilon_{\mathbf{n}, \alpha}-\mu\right)}\right) \phi_{\mathbf{n}, \alpha}^{*} \phi_{\mathbf{n}, \alpha}\right\} \\
& =\prod_{\mathbf{n}, \alpha}\left(1 \mp \mathrm{e}^{-\beta\left(\epsilon_{\mathbf{n}, \alpha}-\mu\right)}\right)^{\mp 1}=\exp \left\{\mp \sum_{\mathbf{n}, \alpha} \ln \left(1 \mp \mathrm{e}^{-\beta\left(\epsilon_{\mathbf{n}, \alpha}-\mu\right)}\right)\right\} .
\end{aligned}
$$

This is the correct result, because from the usual thermodynamic identity $\langle\hat{N}\rangle=\partial \ln Z_{0} / \partial(\beta \mu)$ we find

$$
\langle\hat{N}\rangle=\sum_{\mathbf{n}, \alpha} \frac{1}{\mathrm{e}^{\beta\left(\epsilon_{\mathrm{n}, \alpha}-\mu\right)} \mp 1},
$$

as desired.

\subsubsection{Matsubara expansion}

The second way is easier and more common in practice. We immediately start with

$$
Z_{0}=\int \mathrm{d}\left[\phi^{*}\right] \mathrm{d}[\phi] \mathrm{e}^{-S_{0}\left[\phi^{*}, \phi\right] / \hbar}
$$

and incorporate the boundary conditions by expanding the fields as

$$
\phi_{\alpha}(\mathbf{x}, \tau)=\sum_{\mathbf{n}, n} \phi_{\mathbf{n}, n, \alpha} \chi_{\mathbf{n}}(\mathbf{x}) \frac{\mathrm{e}^{-i \omega_{n} \tau}}{\sqrt{\hbar \beta}}
$$


where $\omega_{n}=\pi(2 n) / \hbar \beta$ for bosons and $\omega_{n}=\pi(2 n+1) / \hbar \beta$ for fermions. These are known as the even and odd Matsubara frequencies, respectively. Using this expansion we have

$$
\begin{aligned}
Z_{0}= & \int\left(\prod_{\mathbf{n}, n, \alpha} \frac{\mathrm{d} \phi_{\mathbf{n}, n, \alpha}^{*} \mathrm{~d} \phi_{\mathbf{n}, n, \alpha}}{(2 \pi i)^{(1+1) / 2}} \frac{1}{(\hbar \beta) \pm 1}\right) \\
& \times \exp \left\{-\frac{1}{\hbar} \sum_{\mathbf{n}, n, \alpha} \phi_{\mathbf{n}, n, \alpha}^{*}\left(-i \hbar \omega_{n}+\epsilon_{\mathbf{n}, \alpha}-\mu\right) \phi_{\mathbf{n}, n, \alpha}\right\},
\end{aligned}
$$

if we also take account of the jacobian involved in the change of integration variables. Note that the difference between the jacobians in the bosonic and fermionic case, is a consequence of the fact that for Grassmamn variables we have that $\int \mathrm{d} \phi f_{2} \phi=f_{2}=\int \mathrm{d}\left(f_{2} \phi\right) f_{2}\left(f_{2} \phi\right)$ instead of the result $\int \mathrm{d}\left(f_{2} \phi\right)\left(1 / f_{2}\right)\left(f_{2} \phi\right)$ that we expect on the basis of ordinary complex integration. Note also that these are all again gaussian integrals, so we find in first instance

$$
\begin{aligned}
Z_{0} & =\prod_{\mathbf{n}, n, \alpha}\left(\beta\left(-i \hbar \omega_{n}+\epsilon_{\mathbf{n}, \alpha}-\mu\right)\right)^{\mp 1} \\
& =\exp \left\{\mp \sum_{\mathbf{n}, n, \alpha} \ln \left(\beta\left(-i \hbar \omega_{n}+\epsilon_{\mathbf{n}, \alpha}-\mu\right)\right)\right\} .
\end{aligned}
$$

To evaluate the sum over Matsubara frequencies we need to add a convergence factor $e^{i \omega_{n} \eta}$ and finally take the limit $\eta \downarrow 0$. The precise reason for this particular procedure cannot be fully understood at this point but is explained in Section 2.5. However, doing so we indeed find that

$$
\lim _{\eta \downarrow 0} \sum_{n} \ln \left(\beta\left(-i \hbar \omega_{n}+\epsilon-\mu\right)\right) \mathrm{e}^{i \omega_{n} \eta}=\ln \left(1 \mp \mathrm{e}^{-\beta(\epsilon-\mu)}\right) .
$$

To see that this is correct, we differentiate the latter equation with respect to $\beta \mu$. This gives us

$$
\lim _{\eta \downarrow 0} \frac{1}{\hbar \beta} \sum_{n} \frac{\mathrm{e}^{i \omega_{n} \eta}}{i \omega_{n}-(\epsilon-\mu) / \hbar}=\mp \frac{1}{\mathrm{e}^{\beta(\epsilon-\mu)} \mp 1},
$$

which can be proved by contour integration in the following way. The function $\hbar \beta /\left(\mathrm{e}^{\hbar \beta z} \mp 1\right)$ has poles at the even and odd Matsubara frequencies with residu \pm 1 . Hence, by Cauchy's theorem the left-hand side is equal to

$$
\lim _{\eta \downarrow 0} \frac{1}{2 \pi i} \int_{\mathrm{c}} \mathrm{d} z \frac{\mathrm{e}^{\eta z}}{z-(\epsilon-\mu) / \hbar} \frac{ \pm 1}{\mathrm{e}^{\hbar \beta z} \mp 1}
$$

with $C$ a contour that fully encloses the imaginary axis in the direction shown in Figure 3. Adding to the contour $C$ the contour $C^{\prime}$, which gives no 


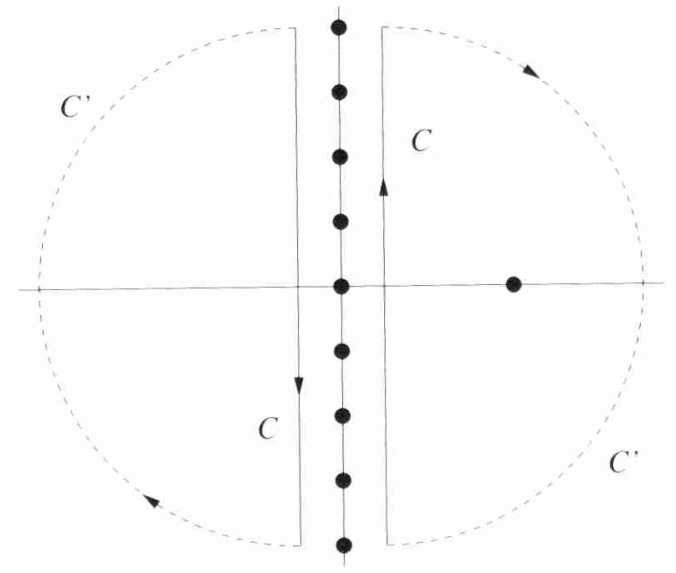

Fig. 3. Illustration of the contour integration that is required to perform the summation over the Matsubara frequencies. The black dots indicate the position of the poles in the integrant.

contribution to the integral, we can again apply Cauchy's theorem to obtain the desired result. Why does the integration over the contour $C^{\prime}$ vanish? The reason for this is that the integrant behaves as $\pm e^{-(\hbar \beta-1) \operatorname{Re}(z)} /|z|$ for $\operatorname{Re}(z) \rightarrow \infty$ and as $-\mathrm{e}^{\eta \operatorname{Re}(z)} /|z|$ for $\operatorname{Re}(z) \rightarrow-\infty$. The integrant thus always vanishes much faster than $1 /|z|$ on the contour $C^{\prime}$ for any $0<\eta<\hbar \beta$.

\subsubsection{Green's function method}

The third and last method is simplest and, therefore, most common in practice. We first rewrite the partition function as

$$
\begin{aligned}
Z_{0}= & \int \mathrm{d}\left[\phi^{*}\right] \mathrm{d}[\phi] \exp \left\{\sum_{\alpha} \int_{0}^{h \beta} \mathrm{d} \tau \int \mathrm{dx}\right. \\
& \left.\times \sum_{\alpha^{\prime}} \int_{0}^{\hbar \beta} \mathrm{d} \tau^{\prime} \int \mathrm{d} \mathbf{x}^{\prime} \phi_{\alpha}^{*}(\mathbf{x}, \tau) G_{\alpha \cdot \alpha^{\prime}}^{-1}\left(\mathbf{x}, \tau ; \mathbf{x}^{\prime}, \tau^{\prime}\right) \phi_{\alpha^{\prime}}\left(\mathbf{x}^{\prime}, \tau^{\prime}\right)\right\}
\end{aligned}
$$

and see $G^{-1}$ as a "matrix" both in spin space as in coordinate space. We then know that this gaussian integral is just

$$
Z_{0}=\left[\operatorname{det}\left(-G^{-1}\right)\right]^{\mp 1}=\exp \left\{\mp \operatorname{Tr}\left[\ln \left(-G^{-1}\right)\right]\right\} .
$$


Clearly, we have from the action $S_{0}\left[\phi^{*}, \phi\right]$ that

$$
\begin{aligned}
G_{\alpha, \alpha^{\prime}}^{-1}\left(\mathbf{x}, \tau ; \mathbf{x}^{\prime}, \tau^{\prime}\right)= & -\frac{1}{\hbar}\left\{\hbar \frac{\partial}{\partial \tau}-\frac{\hbar^{2} \nabla^{2}}{2 m}\right. \\
& \left.+V^{\mathrm{ex}}(\mathbf{x})+\epsilon_{\alpha}-\mu\right\} \delta\left(\mathbf{x}-\mathbf{x}^{\prime}\right) \delta\left(\tau-\tau^{\prime}\right) \delta_{\alpha, \alpha^{\prime}}
\end{aligned}
$$

or equivalently that

$$
\begin{aligned}
\left\{\hbar \frac{\partial}{\partial \tau}-\frac{\hbar^{2} \nabla^{2}}{2 m}+V^{\mathrm{ex}}(\mathbf{x})+\epsilon_{\alpha}-\mu\right\} & G_{\alpha, \alpha^{\prime}}\left(\mathbf{x}, \tau ; \mathbf{x}^{\prime}, \tau^{\prime}\right) \\
& =-h \delta\left(\mathbf{x}-\mathbf{x}^{\prime}\right) \delta\left(\tau-\tau^{\prime}\right) \delta_{\alpha, \alpha^{\prime}}
\end{aligned}
$$

which means that $G_{\alpha \cdot \alpha^{\prime}}\left(\mathbf{x}, \tau ; \mathbf{x}^{\prime}, \tau^{\prime}\right)$ is a Green's function. We come back to its physical meaning shortly. For now we only need to see that the last equation is solved by

$$
\begin{aligned}
& G_{\alpha, \alpha^{\prime}}\left(\mathbf{x}, \tau ; \mathbf{x}^{\prime}, \tau^{\prime}\right)= \\
& \quad=\delta_{\alpha, \alpha^{\prime}} \sum_{\mathbf{n}, n} \frac{-\hbar}{-i \hbar \omega_{n}+\epsilon_{\mathbf{n}, \alpha}-\mu} \chi_{\mathbf{n}}(\mathbf{x}) \chi_{\mathbf{n}}^{*}\left(\mathbf{x}^{\prime}\right) \frac{\mathrm{e}^{-i \omega_{n}\left(\tau-\tau^{\prime}\right)}}{h \beta}
\end{aligned}
$$

thus again

$$
\begin{aligned}
Z_{0} & =\exp \left\{\mp \sum_{\mathbf{n}, n, \alpha} \ln \left(\beta\left(-i \hbar \omega_{n}+\epsilon_{\mathbf{n}, \alpha}-\mu\right)\right)\right\} \\
& =\exp \left\{\mp \sum_{\mathbf{n}, \alpha} \ln \left(1 \mp \mathrm{e}^{-\beta\left(\epsilon_{\mathbf{n}, \alpha}-\mu\right)}\right)\right\} .
\end{aligned}
$$

In principle, we have slightly cheated in the last step of this derivation, because equation (2.65) shows that

$$
G_{\mathbf{n}, n, \alpha ; \mathbf{n}^{\prime}, n^{\prime}, \alpha^{\prime}}=\delta_{\mathbf{n}, \mathbf{n}^{\prime}} \delta_{n, n^{\prime}} \delta_{\alpha, \alpha^{\prime}} \frac{-\hbar}{-i \hbar \omega_{n}+\epsilon_{\mathbf{n}, \alpha}-\mu} .
$$

The problem with this last way of calculating the functional integral is that it does not correctly account for the jacobian involved in the change of variables from imaginary time $\tau$ to the Matsubara frequencies $\omega_{n}$. However, this problem can be avoided by calculating never a single determinant, but always the ratio of two determinants. In this manner the effect of the jacobian is exactly cancelled. We will see some examples of how this works in Sections 2.6 and 2.8.3. 
We now return to the physical meaning of the above Green's function. To see its meaning, we first consider the time-ordered expectation value

$$
\begin{aligned}
& \left\langle T\left[\hat{\psi}_{\alpha}(\mathbf{x}, \tau) \hat{\psi}_{\gamma^{\prime}}^{\dagger}\left(\mathrm{x}^{\prime}, \tau^{\prime}\right)\right]\right\rangle \\
& \quad \equiv \theta\left(\tau-\tau^{\prime}\right)\left\langle\hat{\psi}_{\alpha}(\mathrm{x}, \tau) \hat{\psi}_{\alpha^{\prime}}^{\dagger}\left(\mathbf{x}^{\prime}, \tau^{\prime}\right)\right\rangle \pm \theta\left(\tau^{\prime}-\tau\right)\left\langle\hat{\psi}_{\alpha^{\prime}}^{\dagger}\left(\mathrm{x}^{\prime}, \tau^{\prime}\right) \hat{\psi}_{\alpha}(\mathrm{x}, \tau)\right\rangle .
\end{aligned}
$$

Here the expectation value is taken in the grand-canonical ensemble and $\hat{\psi}_{\alpha}(\mathbf{x} . \tau)$ is the imaginary time Heisenberg operator, which is defined by $c^{(\hat{H}-\mu \hat{N}) \tau / \hbar} \hat{\psi}_{\alpha}(\mathrm{x}) \mathrm{e}^{-(\hat{H}-\mu \hat{N}) \tau / \hbar}$ and therefore obeys the Heisenberg equation of motion $\hbar \partial_{\tau} \hat{\psi}_{\alpha}(\mathrm{x}, \tau)=\left[\hat{H}-\mu \hat{N}, \hat{\psi}_{\alpha}(\mathrm{x}, \tau)\right]_{-}$. For the noninteracting case it reads

$$
\hbar \frac{\partial}{\partial \tau} \hat{\psi}_{\alpha}(\mathbf{x}, \tau)=\left(\frac{\hbar^{2} \boldsymbol{\nabla}^{2}}{2 m}-V^{\mathrm{ex}}(\mathbf{x})-\epsilon_{\alpha}+\mu\right) \hat{\psi}_{\alpha}(\mathbf{x}, \tau) .
$$

As a result,

$$
\begin{array}{r}
\hbar \frac{\partial}{\partial \tau}\left\langle T\left[\hat{\psi}_{\alpha}(\mathbf{x}, \tau) \hat{\psi}_{\alpha^{\prime}}^{\dagger}\left(\mathbf{x}^{\prime} \cdot \tau^{\prime}\right)\right]\right\rangle=\hbar \delta\left(\tau-\tau^{\prime}\right)\left\langle\left[\hat{\psi}_{\alpha}(\mathbf{x}, \tau), \hat{\psi}_{\alpha^{\prime}}^{\dagger}\left(\mathbf{x}^{\prime}, \tau^{\prime}\right)\right]_{\mp}\right\rangle \quad 2.7 \\
+\left(\frac{\hbar^{2} \nabla^{2}}{2 m}-V^{\mathrm{ex}}(\mathbf{x})-\epsilon_{\alpha}+\mu\right)\left\langle T\left[\hat{\psi}_{\alpha}(\mathbf{x}, \tau) \hat{\psi}_{\alpha^{\prime}}^{\dagger}\left(\mathbf{x}^{\prime}, \tau^{\prime}\right)\right]\right\rangle .
\end{array}
$$

Substituting the equal-time relations that we derived in Section 2.1, i.e., $\left[\hat{\psi}_{\alpha}(\mathbf{x}, \tau), \hat{\psi}_{\alpha^{\prime}}^{\dagger}\left(\mathbf{x}^{\prime}, \tau\right)\right]_{\mp}=\delta\left(\mathbf{x}-\mathbf{x}^{\prime}\right) \delta_{\alpha, \alpha^{\prime}}$, thus suggests that

$$
G_{\alpha, \alpha^{\prime}}\left(\mathbf{x}, \tau ; \mathbf{x}^{\prime}, \tau^{\prime}\right)=-\left\langle T\left[\hat{\psi}_{\alpha}(\mathbf{x}, \tau) \hat{\psi}_{\alpha^{\prime}}^{\dagger}\left(\mathbf{x}^{\prime}, \tau^{\prime}\right)\right]\right\rangle .
$$

We can actually also prove this important relation, that bridges the gap between the functional formulation of quantum field theory used here and the more familiar operator formalism. First of all, it is clear from the slicing procedure used in our derivation of the functional integral in Section 2.3, that it automatically leads to time-ordered expectation values. We should, therefore, only be able to prove that

$$
\begin{aligned}
-G_{\alpha, \alpha^{\prime}}\left(\mathbf{x}, \tau ; \mathbf{x}^{\prime}, \tau^{\prime}\right) & =\left\langle\phi_{\alpha}(\mathbf{x}, \tau) \phi_{\alpha^{\prime}}^{*}\left(\mathbf{x}^{\prime}, \tau^{\prime}\right)\right\rangle \\
& \equiv \frac{1}{Z_{0}} \int \mathrm{d}\left[\phi^{*}\right] \mathrm{d}[\phi] \phi_{\alpha}(\mathbf{x}, \tau) \phi_{\alpha^{\prime}}^{*}\left(\mathbf{x}^{\prime}, \tau^{\prime}\right) \mathrm{e}^{-S_{0}\left[\phi^{*}, \phi\right] / \hbar} .
\end{aligned}
$$

This is most easily achieved in the following way. We introduce a partition function in the presence of external currents $J_{\alpha}(\mathbf{x}, \tau)$ and $J_{\alpha}^{*}(\mathbf{x}, \tau)$, where in the fermionic case these currents are also Grassmann variables. The partition function is taken to be

$$
\begin{aligned}
Z_{0}\left[J, J^{*}\right]= & \int \mathrm{d}\left[\phi^{*}\right] \mathrm{d}[\phi] \exp \left\{-\frac{1}{\hbar} S_{0}\left[\phi^{*}, \phi\right]\right. \\
& \left.+\sum_{\alpha} \int_{0}^{h / \beta} \mathrm{d} \tau \int \mathrm{dx}\left(\phi_{\alpha}^{*}(\mathbf{x}, \tau) J_{\alpha}(\mathbf{x}, \tau)+J_{\alpha}^{*}(\mathbf{x}, \tau) \phi_{\alpha}(\mathbf{x}, \tau)\right)\right\},
\end{aligned}
$$


since then we must simply prove that

$$
-G_{\alpha \cdot \alpha^{\prime}}\left(\mathbf{x}, \tau ; \mathbf{x}^{\prime}, \tau^{\prime}\right)=\left.\frac{ \pm 1}{Z_{0}} \frac{\delta^{2} Z_{0}}{\delta \cdot J_{\alpha}^{*}(\mathbf{x}, \tau) \delta \cdot J_{\left(\alpha^{\prime}\right.}\left(\mathbf{x}^{\prime}, \tau^{\prime}\right)}\right|_{J_{.} J^{*}=0} .
$$

Using a short-hand notation we have

$$
Z_{0}\left[J . J^{*}\right]=\int \mathrm{d}\left[\phi^{*}\right] \mathrm{d}[\phi] \exp \left\{\left(\phi\left|G^{-1}\right| \phi\right)+(\phi \mid . J)+(J \mid \phi)\right\} .
$$

The terms in the exponent can be rewritten as $\left(\phi+J G\left|G^{-1}\right| \phi+G J\right)-$ $(J|G| J)$. which is usually called completing the square. Performing a shift in the integration variables, we then casily see that

$$
\begin{aligned}
Z_{0}\left[J, J^{*}\right]= & Z_{0}[0,0] \mathrm{e}^{-(J|G| J)}=Z_{0}[0,0] \exp \left\{-\sum_{\alpha} \int_{0}^{h \beta} \mathrm{d} \tau \int \mathrm{dx}\right. \\
& \left.\times \sum_{\alpha^{\prime}} \int_{0}^{\hbar \beta \beta} \mathrm{d} \tau^{\prime} \int \mathrm{d} \mathbf{x}^{\prime} \cdot J_{\alpha}^{*}(\mathbf{x}, \tau) G_{\alpha, \alpha^{\prime}}\left(\mathbf{x}, \tau ; \mathbf{x}^{\prime}, \tau^{\prime}\right) \cdot J_{\alpha^{\prime}}\left(\mathbf{x}^{\prime}, \tau^{\prime}\right)\right\},
\end{aligned}
$$

which after differentiation indeed leads to the desired result. We can now in fact calculate the expectation value of the time-ordered product of any number of operators. With the above expression for $Z_{0}\left[J, J^{*}\right]$ one can easily prove that this results in the sum of all possible products of time-ordered expectation values of two opcrators. For instance

$$
\begin{aligned}
\left\langle\phi_{\alpha}^{*}(\mathrm{x}, \tau)\right. & \left.\phi_{\alpha^{\prime}}^{*}\left(\mathrm{x}^{\prime}, \tau^{\prime}\right) \phi_{\alpha^{\prime \prime}}\left(\mathrm{x}^{\prime \prime}, \tau^{\prime \prime}\right) \phi_{\alpha^{\prime \prime \prime}}\left(\mathrm{x}^{\prime \prime \prime}, \tau^{\prime \prime \prime}\right)\right\rangle \\
& =\left\langle\phi_{\alpha}^{*}(\mathrm{x}, \tau) \phi_{\alpha^{\prime \prime \prime}}\left(\mathrm{x}^{\prime \prime \prime}, \tau^{\prime \prime \prime}\right)\right\rangle\left\langle\phi_{\alpha^{\prime}}^{*}\left(\mathrm{x}^{\prime}, \tau^{\prime}\right) \phi_{\alpha^{\prime \prime}}\left(\mathrm{x}^{\prime \prime}, \tau^{\prime \prime}\right)\right\rangle \\
& \pm\left\langle\phi_{\alpha}^{*}(\mathrm{x}, \tau) \phi_{\alpha^{\prime \prime}}\left(\mathrm{x}^{\prime \prime}, \tau^{\prime \prime}\right)\right\rangle\left\langle\phi_{\alpha^{\prime}}^{*}\left(\mathrm{x}^{\prime}, \tau^{\prime}\right) \phi_{\alpha^{\prime \prime}}\left(\mathrm{x}^{\prime \prime \prime}, \tau^{\prime \prime \prime}\right)\right\rangle .
\end{aligned}
$$

This is the famous Wick's theorcm, which plays a crucial role in the next section where we start to discuss the profound effects that interactions can have on the results obtained thusfar.

\subsection{Interactions and Feynmann diagrams}

The Green's function, or one-particle propagator, $G_{\alpha, \alpha^{\prime}}\left(\mathrm{x}, \tau ; \mathrm{x}^{\prime}, \tau^{\prime}\right)$ is one of the most important quantities that we want to determine theoretically, because it gives us the possibility to calculate in principle the expectation value of any one-particle observable. It also gives us the elementary excitations of our system of interest. For example, the average density of the spin state $|\alpha\rangle$ is in an ideal gas given by

$$
\begin{aligned}
& \left\langle\hat{\psi}_{\alpha}^{\dagger}(\mathbf{x}, \tau) \hat{\psi}_{\alpha}(\mathbf{x}, \tau)\right\rangle=\mp G_{\alpha, \alpha}\left(\mathbf{x}, \tau ; \mathbf{x}, \tau^{+}\right) \\
& \quad=\lim _{\eta \downarrow 0} \sum_{\mathbf{n}, n} \frac{ \pm \mathrm{e}^{i \omega_{n} \eta}}{\beta\left(-i \hbar \omega_{n}+\epsilon_{\mathbf{n}, \alpha}-\mu\right)}\left|\chi_{\mathbf{n}}(\mathbf{x})\right|^{2}=\sum_{\mathbf{n}} \frac{1}{\mathrm{e}^{\beta\left(\epsilon_{\mathbf{n}, \alpha}-\mu\right)} \mp 1}\left|\chi_{\mathbf{n}}(\mathbf{x})\right|^{2},
\end{aligned}
$$


where we introduced the notation $\tau^{+}$for the limit $\eta \downarrow 0$ of $\tau+\eta$. Note that this procedure is necessary due to the time-ordening involved in the definition of the Green's function and the fact that the field operators do not commute at equal times. It also gives a natural explanation for our previous use of the convergence factor $\mathrm{e}^{i \omega_{n} \eta}$. Moreover, from the argument of the Bose or Fermi distribution function. we see that the elementary excitations have an energy of $\epsilon_{\mathbf{n} . \alpha}-\mu$. If we replace in the Fourier transform of the Green's function, i.e., in

$$
G_{\alpha, \alpha^{\prime}}\left(\mathrm{x}, \mathrm{x}^{\prime} ; i \omega_{n}\right)=-\hbar \delta_{\alpha, \alpha^{\prime}} \sum_{\mathbf{n}} \frac{1}{-i \hbar \omega_{n}+\epsilon_{\mathbf{n}, \alpha}-\mu} \chi_{\mathbf{n}}(\mathbf{x}) \chi_{\mathbf{n}}^{*}\left(\mathbf{x}^{\prime}\right),
$$

$i \omega_{n}$ by $\omega$ we also see that $G_{\alpha \cdot \alpha^{\prime}}\left(\mathbf{x}, \mathbf{x}^{\prime} ; \omega\right)$ has a pole at $\hbar \omega=\epsilon_{\mathbf{n}, \alpha}-\mu$. This is a general result: Poles in $G_{\alpha, \alpha^{\prime}}\left(\mathrm{x}, \mathrm{x}^{\prime} ; \omega\right)$ correspond to the energies of the elementary excitations. These energies in general can also have a negative imaginary components, which then correspond to the lifetime of the excitation. The question that arises, therefore, is how to determine also the Green's function for an interacting system.

This can be done in perturbation theory, as follows. We want to calculate the expectation value

$$
\begin{aligned}
-\left\langle\phi_{\alpha}(\mathrm{x}, \tau) \phi_{\alpha^{\prime}}^{*}\left(\mathrm{x}^{\prime}, \tau^{\prime}\right)\right\rangle & = \\
- & \frac{1}{Z} \int \mathrm{d}\left[\phi^{*}\right] \mathrm{d}[\phi] \phi_{\alpha}(\mathbf{x}, \tau) \phi_{\alpha^{\prime}}^{*}\left(\mathbf{x}^{\prime}, \tau^{\prime}\right) \mathrm{e}^{-S\left[\phi^{*}, \phi\right] / h}
\end{aligned}
$$

with the action $S\left[\phi^{*}, \phi\right]=S_{0}\left[\phi^{*}, \phi\right]+S_{\text {int }}\left[\phi^{*}, \phi\right]$. We now expand both the numerator and the denominator in powers of $S_{\text {int }}$. Up to first order we find for the partition function

$$
\begin{aligned}
Z & =\int \mathrm{d}\left[\phi^{*}\right] \mathrm{d}[\phi] \mathrm{e}^{-S_{0}\left[\phi^{*}, \phi\right] / \hbar}\left(1-\frac{S_{\text {int }}\left[\phi^{*}, \phi\right]}{\hbar}\right) \\
& \equiv Z_{0}\left(1-\frac{1}{\hbar}\left\langle S_{\text {int }}\left[\phi^{*}, \phi\right]\right\rangle_{0}\right)
\end{aligned}
$$

Using Wick's theorem, we thus have

$$
\begin{aligned}
-\frac{1}{\hbar}\left\langle S_{\mathrm{int}}\left[\phi^{*}, \phi\right]\right\rangle_{0}= & \frac{1}{2} \sum_{\alpha, \alpha^{\prime}} \int_{0}^{h \beta} \mathrm{d} \tau \int \mathrm{d} \mathbf{x} \int \mathrm{d} \mathbf{x}^{\prime} G_{0 ; \alpha, \alpha}\left(\mathbf{x}, \tau ; \mathbf{x}, \tau^{+}\right) \\
& \times \frac{-V\left(\mathbf{x}-\mathbf{x}^{\prime}\right)}{\hbar} G_{0 ; \alpha^{\prime}, \alpha^{\prime}}\left(\mathbf{x}^{\prime}, \tau ; \mathbf{x}^{\prime}, \tau^{+}\right) \\
& \pm \frac{1}{2} \sum_{\alpha} \int_{0}^{\hbar \beta} \mathrm{d} \tau \int \mathrm{d} \mathbf{x} \int \mathrm{d} \mathbf{x}^{\prime} G_{0 ; \alpha, \alpha}\left(\mathbf{x}^{\prime}, \tau ; \mathbf{x}, \tau^{+}\right) \\
& \times \frac{-V\left(\mathbf{x}-\mathbf{x}^{\prime}\right)}{\hbar} G_{0 ; \alpha, \alpha}\left(\mathbf{x}, \tau ; \mathbf{x}^{\prime}, \tau^{+}\right) .
\end{aligned}
$$




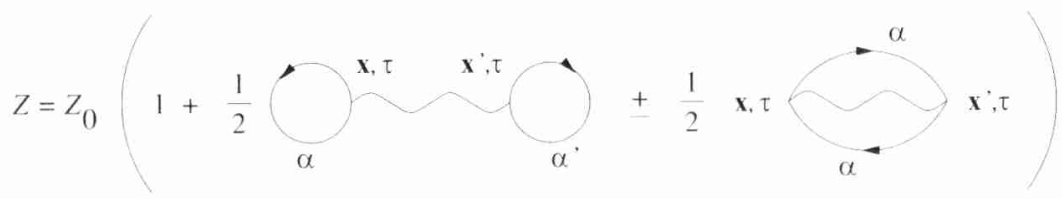

Fig. 4. Diagrammatic representation of the partition function up to first order in the interaction.
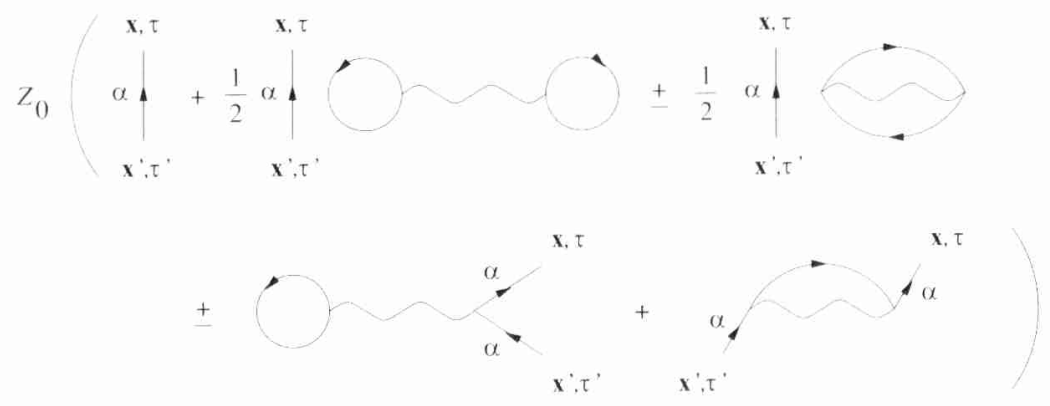

Fig. 5. Diagrammatic representation of the numerator of equation (2.80) up to first order in the interaction.

To understand the general structure of the perturbation expansion, it is very convenient to represent equations (2.81) and (2.82) in terms of Feynman diagrams. The final result is shown in Figure 4, where a wiggly line corresponds to the factor $-V\left(\mathbf{x}-\mathbf{x}^{\prime}\right) / \hbar$ and a thin arrowed line pointing from $\left(\mathrm{x}^{\prime}, \tau^{\prime}\right)$ to $(\mathrm{x}, \tau)$ represents the noninteracting Green's function $G_{0: \alpha, \alpha}\left(\mathbf{x}, \tau ; \mathbf{x}^{\prime}, \tau^{\prime}\right)$. In Figure 4 we have, for clarity sake, also explicitly indicated the various coordinates and spin degrees of freedom that we have to either integrate or sum over, respectively. It is, however, much more common in practice to suppress in Feynman diagrams those degrees of freedom that have to be integrated or summed over, and denote only the degrees of freedom on which the quantity of interest depends explicitly. For example, for the numerator of equation (2.80) we obtain

$$
\begin{gathered}
-\int \mathrm{d}\left[\phi^{*}\right] \mathrm{d}[\phi] \phi_{\alpha}(\mathbf{x}, \tau) \phi_{\alpha^{\prime}}^{*}\left(\mathbf{x}^{\prime}, \tau^{\prime}\right) \mathrm{e}^{-S_{0}\left[\phi^{*}, \phi\right] / \hbar}\left(1-\frac{S_{\mathrm{int}}\left[\phi^{*}, \phi\right]}{\hbar}\right) \\
=Z_{0}\left(-\left\langle\phi_{\alpha}(\mathbf{x}, \tau) \phi_{\alpha^{\prime}}^{*}\left(\mathbf{x}^{\prime}, \tau^{\prime}\right)\right\rangle_{0}+\frac{1}{\hbar}\left\langle\phi_{\alpha}(\mathbf{x}, \tau) \phi_{\alpha^{\prime}}^{*}\left(\mathbf{x}^{\prime}, \tau^{\prime}\right) S_{\text {int }}\left[\phi^{*}, \phi\right]\right\rangle_{0}\right),
\end{gathered}
$$

whose diagrammatic equivalent is then given in Figure 5. Up to first order the interacting Green's function, which is represented by a thick arrowed line, obeys the equation shown in Figure 6. Note that the so-called disconnected diagrams have exactly cancelled. This is a general feature that 


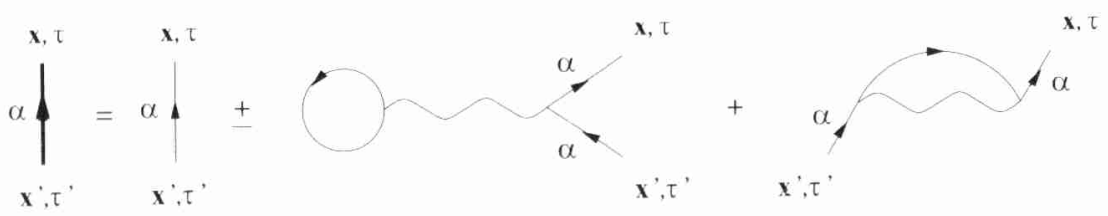

Fig. 6. Interacting Green's function up to first order in the interaction.

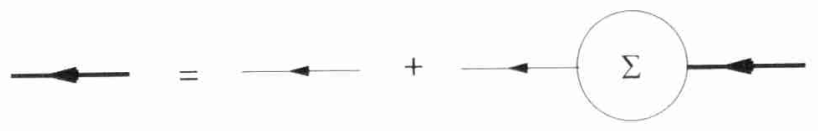

Fig. 7. Exact Dyson equation for the interacting Green's function.

happens for any expectation value, i.e., the disconnected diagrams that occur in the numerator are always exactly cancelled by the denominator $[18,19]$.

One can also show that the general structure of the perturbation expansion of the Green's function is as in Figure 7, in which only one-particle irreducible diagrams contribute to the selfenergy $h \Sigma$. These one-particle irreducible diagrams can be distinguished from the fact that they do not become disconnected if we cut a single thin arrowed line. This is Dyson's equation [20]. It is particularly insightful for a homogeneous gas or for a trapped gas in the weak-coupling limit, since then the one-particle states $\chi_{\mathbf{n}}(\mathbf{x})$ are not affected by the interactions and the exact one-particle propagator can be written as

$$
G_{\alpha, \alpha^{\prime}}\left(\mathbf{x}, \tau ; \mathbf{x}^{\prime}, \tau^{\prime}\right)=\sum_{\mathbf{n}, n} G_{\alpha, \alpha^{\prime}}\left(\mathbf{n}, i \omega_{n}\right) \chi_{\mathbf{n}}(\mathbf{x}) \chi_{\mathbf{n}}^{*}\left(\mathbf{x}^{\prime}\right) \frac{\mathrm{e}^{-i \omega_{n}\left(\tau-\tau^{\prime}\right)}}{\hbar \beta} .
$$

Note that for realistic trapped atomic gases this weak-coupling limit is essentially always realized in the normal phase of the gas. It is, therefore, also of some experimental interest to consider that limit in more detail first.

One then finds that the Dyson equation in first instance becomes

$$
\begin{aligned}
G_{\alpha, \alpha^{\prime}}\left(\mathbf{n}, i \omega_{n}\right)= & G_{0 ; \alpha, \alpha^{\prime}}\left(\mathbf{n}, i \omega_{n}\right) \\
& +\sum_{\alpha^{\prime \prime}, \alpha^{\prime \prime \prime}} G_{0 ; \alpha, \alpha^{\prime \prime}}\left(\mathbf{n}, i \omega_{n}\right) \Sigma_{\alpha^{\prime \prime}, \alpha^{\prime \prime \prime}}\left(\mathbf{n}, i \omega_{n}\right) G_{\alpha^{\prime \prime \prime}, \alpha^{\prime}}\left(\mathbf{n}, i \omega_{n}\right) .
\end{aligned}
$$

Because $G_{0 ; \alpha, \alpha^{\prime}}\left(\mathbf{n}, i \omega_{n}\right)=G_{0 ; \alpha}\left(\mathbf{n}, i \omega_{n}\right) \delta_{\alpha, \alpha^{\prime}}$ and because we have assumed the interactions to be spin independent, we can easily convince ourselves that in perturbation theory it is always true that the selfenergy obeys $\Sigma_{\alpha^{\prime \prime}, \alpha^{\prime \prime \prime}}\left(\mathbf{n}, i \omega_{n}\right)=\Sigma_{\alpha^{\prime \prime}}\left(\mathbf{n}, i \omega_{n}\right) \delta_{\alpha^{\prime \prime}, \alpha^{\prime \prime \prime}}$ and as a result also that up to all orders in the interaction $G_{\alpha, \alpha^{\prime}}\left(\mathbf{n}, i \omega_{n}\right)=G_{\alpha}\left(\mathbf{n}, i \omega_{n}\right) \delta_{\alpha, \alpha^{\prime}}$. As we will see 


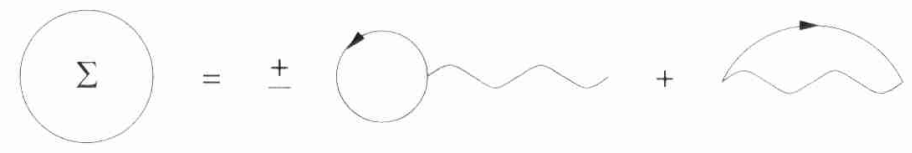

Fig. 8. Selfenergy up to first order in the interaction.

in Section 2.6, however, nonperturbative effect can change this result due to the phenomena of spontaneous broken symmetry. Ignoring the latter for the moment, we find for each spin state the uncoupled equation

$$
G_{\alpha}\left(\mathbf{n}, i \omega_{n}\right)=G_{0 ; \alpha}\left(\mathbf{n}, i \omega_{n}\right)+G_{0 ; \alpha}\left(\mathbf{n}, i \omega_{n}\right) \Sigma_{\alpha}\left(\mathbf{n}, i \omega_{n}\right) G_{\alpha}\left(\mathbf{n}, i \omega_{n}\right),
$$

or

$$
\begin{aligned}
\frac{1}{G_{\alpha}\left(\mathbf{n}, i \omega_{n}\right)} & =\frac{1}{G_{0 ; \alpha}\left(\mathbf{n}, i \omega_{n}\right)}-\Sigma_{\alpha \chi}\left(\mathbf{n}, i \omega_{n}\right) \\
& =-\frac{1}{\hbar}\left(-i \hbar \omega_{n}+\epsilon_{\mathbf{n}, \alpha}-\mu\right)-\Sigma_{\alpha}\left(\mathbf{n}, i \omega_{n}\right) .
\end{aligned}
$$

Hence

$$
G_{\alpha}\left(\mathbf{n}, i \omega_{n}\right)=\frac{-\hbar}{-i \hbar \omega_{n}+\epsilon_{\mathbf{n}, \alpha}+\hbar \Sigma_{\alpha}\left(\mathbf{n}, i \omega_{n}\right)-\mu},
$$

which shows that the poles in the Green's function are indeed shifted by the interactions.

In our lowest order calculation we have found for the selfenergy the diagrammatic result shown in Figure 8. This is the most simple approximation we can think of. In the weak-coupling limit it is mathematically equal to

$$
\begin{aligned}
\hbar \Sigma_{\alpha}\left(\mathbf{n}, i \omega_{n}\right)= & \sum_{\mathbf{n}^{\prime}, \alpha^{\prime}} V_{\mathbf{n}, \mathbf{n}^{\prime} ; \mathbf{n}, \mathbf{n}^{\prime}} \frac{1}{\mathrm{e}^{\beta\left(\epsilon_{\mathbf{n}^{\prime}, \alpha^{\prime}}-\mu\right)} \mp 1} \\
& \mp \sum_{\mathbf{n}^{\prime}} V_{\mathbf{n}^{\prime}, \mathbf{n} ; \mathbf{n}, \mathbf{n}^{\prime}} \frac{1}{\mathrm{e}^{\beta\left(\epsilon_{\mathbf{n}^{\prime}, \alpha}-\mu\right)} \mp 1} \\
= & \sum_{\mathbf{n}^{\prime}, \alpha^{\prime}}\left(V_{\mathbf{n}, \mathbf{n}^{\prime} ; \mathbf{n}, \mathbf{n}^{\prime}} \pm V_{\left.\mathbf{n}^{\prime}, \mathbf{n} ; \mathbf{n}, \mathbf{n}^{\prime} \delta_{\alpha, \alpha^{\prime}}\right)} \frac{1}{\mathrm{e}^{\beta\left(\epsilon_{\mathbf{n}^{\prime}, \alpha^{\prime}}-\mu\right)} \mp 1} .\right.
\end{aligned}
$$

The first term is known as the direct or Hartree, and the second term as the exchange or Fock contribution to the selfenergy. From equation (2.89) we conclude that the matrix elements of the interaction enter only in the combination $V_{\mathbf{n}, \mathbf{n}^{\prime} ; \mathbf{n}, \mathbf{n}^{\prime}} \pm V_{\mathbf{n}^{\prime}, \mathbf{n} ; \mathbf{n}, \mathbf{n}^{\prime}} \delta_{\alpha, \alpha^{\prime}}$. Clearly, this is a refection of the Pauli principle, which forces the effective interaction between two fermionic atoms in the same state $|\mathbf{n}, \alpha\rangle$ to vanish. 


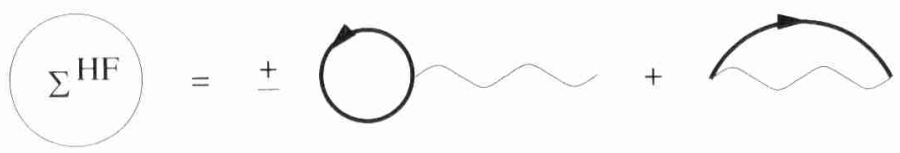

Fig. 9. Hartree Fock approximation for the selfenergy.

To make the theory selfcomsistent we should use in the expression for the selfenergy not the noninteracting propegators, but precisely those that follow from the Dyson equation. This is the Hartree Fock approximation. which is diagrammatically given in Figure 9. It leads in the weak-compling limit to the new dispersion relation $\hbar \omega_{\mathbf{n}, \alpha}=\epsilon_{\mathbf{n}, \alpha}+\hbar \Sigma_{\alpha}^{\mathrm{HF}}(\mathbf{n}, 0)-\mu$ for the "dressed" particles or quasiparticles of the gas, where the selfenergy is essentially also found from equation (2.89). We only have to replace in the right-hand side $\epsilon_{\mathbf{n}^{\prime}} \cdot \alpha^{\prime}-\mu$ by $\epsilon_{\mathbf{n}^{\prime}, \alpha^{\prime}}+\hbar \Sigma_{\alpha^{\prime}}^{\mathrm{HF}}\left(\mathbf{n}^{\prime}, 0\right)-\mu$. In this manner we have thus obtained an approximation to the interacting Green's function that is nomperturbative in the interaction and effectively sums an infinite number of Feymman diagrams. Of course, the Hartree Fock approximation can also be used in the strong-coupling limit. In that case it diagrammatically still corresponds to the solution of the Dyson equation of Figure 7 , with a selfenergy as shown in Figure 9. However, we are then no longer allowed to assume that the exact Green's function is diagonal in the eigenstates $\chi_{\mathbf{n}}(\mathbf{x})$ of the external trapping potential. To see more explicitly what this means physically, it is convenient to rederive the Hartree Fock theory without making use of perturbation theory.

\subsection{Hartree-Fock theory for an atomic Fermi gas}

Because the Hartree Fock theory is very useful in a large number of circumstances, and because we want to illustrate a very useful technique which is nowadays often used in the literature, we are now going to reproduce the above results without making use of a diagramatic expansion. We consider for simplicity a fermionic mixture with an equal number of atoms in two hyperfine states and start by splitting our spin-independent interaction $V\left(\mathrm{x}-\mathrm{x}^{\prime}\right)$ into two spin-dependent parts such that one contributes only to the Hartree diagram and the other only to the Fock diagram. Denoting a spin-dependent interaction by $V_{\alpha^{\prime}, \beta^{\prime}: \alpha, \beta}\left(\mathrm{x}-\mathrm{x}^{\prime}\right)$ we thus want that

$$
V\left(\mathbf{x}-\mathbf{x}^{\prime}\right) \delta_{\alpha, \alpha^{\prime}} \delta_{\beta, \beta^{\prime}}=V_{\alpha^{\prime}, \beta^{\prime} ; \alpha, \beta}^{\mathrm{H}}\left(\mathbf{x}-\mathbf{x}^{\prime}\right)+V_{\alpha^{\prime}, \beta^{\prime}: \alpha, \beta}^{\mathrm{F}}\left(\mathbf{x}-\mathbf{x}^{\prime}\right)
$$

with $\sum_{\beta} V_{\beta, \alpha ; \alpha, \beta}^{\mathrm{H}}\left(\mathrm{x}-\mathrm{x}^{\prime}\right)=\sum_{\beta} V_{\alpha, \beta ; \alpha, \beta}^{\mathrm{F}}\left(\mathrm{x}-\mathrm{x}^{\prime}\right)=0$. Using operators in spin space, a possible solution to these equations is

$$
\hat{V}^{\mathrm{H}}=\frac{2}{3}\left(2-\hat{P}_{12}\right) V\left(\mathbf{x}-\mathrm{x}^{\prime}\right)
$$


and

$$
\hat{V}^{\mathrm{F}}=\frac{1}{3}\left(2 \hat{P}_{12}-1\right) V\left(\mathrm{x}-\mathrm{x}^{\prime}\right) .
$$

where $\hat{P}_{12}=\left(1+\hat{\boldsymbol{\sigma}}_{1} \cdot \hat{\boldsymbol{\sigma}}_{2}\right) / 2$ is the exchange operator of the spins of atoms 1 and 2 , and $\sigma_{\alpha, \alpha^{\prime}}$ are the usual Pauli matrices. Clearly, we now have

$$
\begin{aligned}
S_{\text {int }}\left[\phi^{*}, \phi\right]= & \frac{1}{2} \sum_{\alpha, \alpha^{\prime}: \beta, \beta^{\prime}} \int_{0}^{\hbar, \beta} \mathrm{d} \tau \int \mathrm{d} \mathbf{x} \int \mathrm{d} \mathbf{x}^{\prime} \\
& \times\left\{\phi_{\alpha^{\prime}}^{*}(\mathbf{x}, \tau) \phi_{\alpha}(\mathbf{x}, \tau) V_{\alpha^{\prime}, \beta^{\prime}: \alpha, \beta}^{\mathrm{H}}\left(\mathbf{x}-\mathbf{x}^{\prime}\right) \phi_{\beta^{\prime}}^{*}\left(\mathbf{x}^{\prime}, \tau\right) \phi_{\beta}\left(\mathbf{x}^{\prime}, \tau\right)\right. \\
& \left.-\phi_{\alpha^{\prime}}^{*}(\mathbf{x}, \tau) \phi_{\beta}\left(\mathbf{x}^{\prime}, \tau\right) V_{\alpha^{\prime}, \beta^{\prime} ; \alpha, \beta}^{\mathrm{F}}\left(\mathbf{x}-\mathbf{x}^{\prime}\right) \phi_{\beta^{\prime}}^{*}\left(\mathbf{x}^{\prime}, \tau\right) \phi_{\alpha}(\mathbf{x}, \tau)\right\},
\end{aligned}
$$

which we write as

$$
S_{\text {int }}\left[\phi^{*}, \phi\right] \equiv \frac{1}{2}\left(\phi^{*} \phi\left|V^{\mathrm{H}}\right| \phi^{*} \phi\right)-\frac{1}{2}\left(\phi^{*} \phi|| V^{\mathrm{F}} \| \phi^{*} \phi\right) .
$$

We next apply a so-called Hubbard-Stratonovich transformation $[21,22]$ to both the Hartree and the Fock parts of the interaction. First the Hartree part.

We note that $\mathrm{e}^{-S_{\mathrm{int}}^{\mathrm{H}}\left[\phi^{*}, \phi\right]}$ can be written as a functional integral over the four real fields contained in $\kappa_{\alpha, \alpha^{\prime}}(\mathbf{x}, \tau) \equiv \kappa_{0}(\mathbf{x}, \tau) \delta_{\alpha, \alpha^{\prime}}+\kappa(\mathbf{x}, \tau) \cdot \boldsymbol{\sigma}_{\alpha, \alpha \gamma^{\prime}}$ as

$$
\begin{aligned}
& \exp \left\{-\frac{1}{2 \hbar}\left(\phi^{*} \phi\left|V^{\mathrm{H}}\right| \phi^{*} \phi\right)\right\}= \\
& \quad \int \mathrm{d}[\kappa] \exp \left\{\frac{1}{2 \hbar}\left(\kappa\left|V^{\mathrm{H}-1}\right| \kappa\right)-\frac{1}{2 \hbar}\left(\kappa \mid \phi^{*} \phi\right)-\frac{1}{2 \hbar}\left(\phi^{*} \phi \mid \kappa\right)\right\} .
\end{aligned}
$$

If we put $S_{\text {int }}^{\mathrm{F}}\left[\phi^{*}, \phi\right]=0$ for a moment, we can substitute this equality in the partition function to obtain $Z=\int \mathrm{d}[\kappa] \mathrm{d}\left[\phi^{*}\right] \mathrm{d}[\phi] \mathrm{e}^{-S\left[\kappa, \phi^{*}, \phi\right] / h}$ with the action

$$
\begin{aligned}
S\left[\kappa, \phi^{*}, \phi\right]= & -\frac{1}{2}\left(\kappa\left|V^{\mathrm{H}^{-1}}\right| \kappa\right)+\sum_{\alpha, \alpha^{\prime}} \int_{0}^{h \beta} \mathrm{d} \tau \int \mathrm{dx} \phi_{\alpha}^{*}(\mathrm{x}, \tau)\left\{\left(\hbar \frac{\partial}{\partial \tau} \quad(2.96)\right.\right. \\
& \left.\left.-\frac{\hbar^{2} \nabla^{2}}{2 m}+V^{\mathrm{ex}}(\mathrm{x})+\epsilon_{\alpha}-\mu\right) \delta_{\alpha, \alpha^{\prime}}+\kappa_{\alpha, \alpha^{\prime}}(\mathbf{x}, \tau)\right\} \phi_{\alpha^{\prime}}(\mathbf{x}, \tau) .
\end{aligned}
$$

We see that in this manner the action for the fermions has become quadratic with a selfenergy $\hbar \Sigma_{\alpha, \alpha^{\prime}}\left(\mathbf{x}, \tau ; \mathbf{x}^{\prime}, \tau^{\prime}\right)=\kappa_{\alpha, \alpha^{\prime}}(\mathbf{x}, \tau) \delta\left(\mathbf{x}-\mathbf{x}^{\prime}\right) \delta\left(\tau-\tau^{\prime}\right)$. Hence, we can now integrate out the fermion fields to obtain $Z=\int \mathrm{d}[\kappa] \mathrm{e}^{-S^{\text {eff }}[\kappa] / \hbar}$ and

$$
S^{\text {eff }}[\kappa]=-\frac{1}{2}\left(\kappa\left|V^{\mathrm{H}^{-1}}\right| \kappa\right)-\hbar \operatorname{Tr}\left[\ln \left(-G^{-1}\right)\right],
$$


with $G^{-1}=G_{0}^{-1}-\Sigma$ also a functional of $\kappa_{\alpha, \alpha^{\prime}}(\mathrm{x}, \tau)$. Up to now we have not made any approximations and have only performed an exact rewriting of the partition function. However, the action $S^{\text {eff }}[\kappa]$ contains all powers of the fields $\kappa_{\alpha, \alpha^{\prime}}(\mathbf{x}, \tau)$ and is thus quite complicated. It may therefore appear that we have not made any progress.

This is not the case, because the partition function will be largest for configurations that minimize the action $S^{\mathrm{eff}}[\kappa]$. To make use of this observation. we expand the action around its minimum, i.e., we put $\kappa_{\alpha, \alpha^{\prime}}(\mathrm{x}, \tau)=$ $\left\langle\kappa_{\alpha, \alpha^{\prime}}(\mathrm{x})\right\rangle+\delta \kappa_{\alpha, \alpha^{\prime}}(\mathrm{x}, \tau)$ and require that $\delta S^{\mathrm{eff}}[\kappa] /\left.\delta \kappa_{\alpha, \alpha^{\prime}}(\mathrm{x}, \tau)\right|_{\kappa=\langle\kappa\rangle}=0$. If we neglect the fluctuations, we find that $Z \simeq e^{-S^{\mathrm{fff}}[\langle\kappa\rangle] / \hbar}$. This will turn out to be the Hartree approximation, as we will show now. First of all we have

$$
G^{-1}=G_{0}^{-1}-\langle\kappa\rangle / \hbar-\delta \hbar / \hbar \equiv G^{\mathrm{H}^{-1}}-\delta \kappa / \hbar=G^{\mathrm{H}^{-1}}\left(1-G^{\mathrm{H}} \delta \kappa / \hbar\right) .
$$

Substituting this in equation (2.97) gives us for the terms linear in the fluctuations

$$
-\hbar \operatorname{Tr}\left[-G^{\mathrm{H}} \delta \kappa / \hbar\right]-\left(\delta \kappa\left|V^{\mathrm{H}^{-1}}\right|\langle\kappa\rangle\right)
$$

If $\left\langle\kappa_{\alpha, \sigma^{\prime}}(x)\right\rangle$ is indecd a minimum of the action $S^{\mathrm{eff}}[\kappa]$, these linear terms have to vanish, which implies that

$$
\left\langle\kappa_{\alpha^{\prime}, \alpha}(\mathbf{x})\right\rangle=\sum_{\beta, \beta^{\prime}} \int \mathrm{d} \mathbf{x} V_{\alpha^{\prime}, \beta^{\prime} ; \alpha \cdot \beta}^{\mathrm{H}}\left(\mathbf{x}-\mathrm{x}^{\prime}\right) G_{\beta, \beta^{\prime}}^{\mathrm{H}}\left(\mathrm{x}^{\prime}, \tau ; \mathbf{x}^{\prime}, \tau^{+}\right) .
$$

As promised, this is precisely the most general mathematical expression for the Hartree contribution to the selfenergy in Figure 9. Taking again $\left\langle\kappa_{\alpha \alpha^{\prime}, \alpha}(\mathrm{x})\right\rangle=\kappa_{i}(\mathrm{x}) \delta_{\alpha^{\prime}, \alpha}$, we also have that the Hartree approximation to the one-particle propagator obeys $G_{\beta, \beta^{\prime}}^{\mathrm{H}}\left(\mathbf{x}^{\prime}, \tau ; \mathbf{x}^{\prime}, \tau^{+}\right)=n\left(\mathbf{x}^{\prime}\right) \delta_{\beta, \beta^{\prime}} / 2$, with $n\left(\mathbf{x}^{\prime}\right)$ the average total atomic density, and we obtain the usual Hartree selfenergy

$$
\mathrm{fi}(\mathrm{x})=\sum_{\beta} \int \mathrm{d} \mathrm{x}^{\prime} V_{\alpha, \beta ; \alpha, \beta}^{\mathrm{H}}\left(\mathbf{x}-\mathrm{x}^{\prime}\right) \frac{n\left(\mathrm{x}^{\prime}\right)}{2}=\int \mathrm{d} \mathrm{x}^{\prime} V\left(\mathbf{x}-\mathbf{x}^{\prime}\right) n\left(\mathbf{x}^{\prime}\right) .
$$

Now we again want to include the Fock part of the interaction and treat this also by a Hubbard-Stratonovich transformation. This requires introducing four real ficlds that depend on two spatial coordinates. They are denoted by $\lambda_{\alpha, \alpha^{\prime}}\left(\mathbf{x}, \mathbf{x}^{\prime}, \tau\right)$. Schematically we then use

$$
\begin{aligned}
& \exp \left\{\frac{1}{2 \hbar}\left(\phi^{*} \phi\left\|V^{\mathrm{F}}\right\| \phi^{*} \phi\right)\right\} \\
& =\int \mathrm{d}[\lambda] \exp \left\{-\frac{1}{2 \hbar}\left(\lambda\left\|V^{\mathrm{F}-1}\right\| \lambda\right)+\frac{1}{2 \hbar}\left(\lambda \| \phi^{*} \phi\right)+\frac{1}{2 \hbar}\left(\phi^{*} \phi \| \lambda\right)\right\},
\end{aligned}
$$


which leads to the total selfenergy

$$
\hbar \Sigma_{\alpha, \alpha^{\prime}}\left(\mathbf{x} \cdot \tau: \mathbf{x}^{\prime} \cdot \tau^{\prime}\right)=\left[\kappa_{\alpha, \alpha^{\prime}}(\mathbf{x}, \tau) \delta\left(\mathbf{x}-\mathbf{x}^{\prime}\right)-\lambda_{\alpha, \alpha^{\prime}}\left(\mathbf{x}, \mathbf{x}^{\prime}, \tau\right)\right] \delta\left(\tau-\tau^{\prime}\right)
$$

and after integration over the fermion fields to the effective action

$$
S^{\mathrm{eff}}[\kappa, \lambda]=-\frac{1}{2}\left(\kappa\left|V^{\mathrm{H}-1}\right| \kappa\right)+\frac{1}{2}\left(\lambda|| V^{\mathrm{F}-1}|| \lambda\right)-\hbar \operatorname{Tr}\left[\ln \left(-G^{-1}\right)\right] .
$$

Requiring now that also $\delta S^{\mathrm{eff}}[\kappa, \lambda] /\left.\delta \lambda_{\alpha, \alpha^{\prime}}\left(\mathrm{x}, \mathbf{x}^{\prime}, \tau\right)\right|_{\lambda=\langle\lambda\rangle}=0$, then leads to the expected Fock selfenergy

$$
\left\langle\lambda_{\alpha^{\prime}, \alpha}\left(\mathrm{x}^{\prime}, \mathrm{x}\right)\right\rangle=\sum_{\beta, \beta^{\prime}} \int \mathrm{d} \mathrm{x}^{\prime} V_{\beta^{\prime}, \alpha^{\prime}: \alpha, \beta}^{\mathrm{F}}\left(\mathrm{x}-\mathrm{x}^{\prime}\right) G_{\beta^{\prime}, \beta}^{\mathrm{HF}}\left(\mathrm{x}^{\prime}, \tau: \mathrm{x}, \tau^{+}\right) .
$$

In the symmetric case it simply becomes $\left\langle\lambda_{\alpha^{\prime}, \alpha}\left(\mathbf{x}^{\prime}, \mathbf{x}\right)\right\rangle=\lambda\left(\mathbf{x}^{\prime}, \mathbf{x}\right) \delta_{\alpha^{\prime}, \alpha}$ with

$$
\lambda\left(\mathrm{x}^{\prime}, \mathrm{x}\right)=V\left(\mathrm{x}-\mathrm{x}^{\prime}\right) n\left(\mathrm{x}^{\prime}, \mathrm{x}\right)
$$

and $n\left(\mathbf{x}^{\prime}, \mathbf{x}\right)=\sum_{\beta} G_{\beta, \beta}^{\mathrm{HF}}\left(\mathrm{x}^{\prime} \cdot \tau: \mathbf{x} \cdot \tau^{+}\right) / 2=\sum_{\beta}\left\langle\psi_{\beta}^{\dagger}(\mathrm{x}, \tau) \psi_{\beta}\left(\mathrm{x}^{\prime}, \tau\right)\right\rangle / 2$ exactly the Hartree-Fock approximat ion to the off-diagonal part of the one-particle density matrix.

Note that to actually perfurm the Hartree Fock calculation in the latter case, we need to be able to determine the Green's function $G_{\alpha, \alpha^{\prime}}^{\mathrm{HF}}\left(\mathrm{x}, \tau ; \mathrm{x}^{\prime}, \tau^{\prime}\right)$. The easiest way to do so, is by realizing that it is the Green's function of the operator in the fermionic piece of the action $S\left[\kappa, \lambda, \phi^{*}, \phi\right]$ obtained after the Hubbard-Stratonovich transformations. If we diagonalize this operator by solving the eigenvalue problem

$$
\left\{-\frac{\hbar^{2} \nabla^{2}}{2 m}+V^{\mathrm{ex}}(\mathrm{x})+\kappa(\mathrm{x})-\epsilon_{\mathbf{n}}^{\prime}\right\} \chi_{\mathbf{n}}^{\prime}(\mathrm{x})-\int \mathrm{d} \mathrm{x}^{\prime} \lambda\left(\mathrm{x}, \mathrm{x}^{\prime}\right) \chi_{\mathbf{n}}^{\prime}\left(\mathrm{x}^{\prime}\right)=0,
$$

the desired one-particle propagator aquires the ideal gas form

$$
G_{\alpha, \alpha^{\prime}}^{\mathrm{HF}}\left(\mathbf{x}, \tau ; \mathbf{x}^{\prime}, \tau^{\prime}\right)=\delta_{\alpha, \alpha^{\prime}} \sum_{\mathbf{n}, n} \frac{-\hbar}{-i \hbar \omega_{n}+\epsilon_{\mathbf{n}, \alpha}^{\prime}-\mu} \chi_{\mathbf{n}}^{\prime}(\mathbf{x}) \chi_{\mathbf{n}}^{\prime *}\left(\mathbf{x}^{\prime}\right) \frac{\mathrm{e}^{-i \omega_{n}\left(\tau-\tau^{\prime}\right)}}{\hbar \beta},
$$

with new one-particle energies $\epsilon_{\mathbf{n}, \alpha}^{\prime}=\epsilon_{\mathbf{n}}^{\prime}+\epsilon_{\alpha}$ and eigenstates $\chi_{\mathbf{n}}^{\prime}(\mathbf{x})$ that incorporate the average effect of the interactions of an atom with all the other atoms in the gas. Clearly, in the cases that the eigenstates are not affected by these so-called mean-field effects, we recover the weak-coupling results of the previous section. 
Although we have thus precisely reproduced our diagrammatic result. there are two important advantages in using the Hubbard-Stratonovich transformation. First, it is in principle exact, and allows us to also calculate corrections to the Hartree Fock approximation. For example, if we expand $S^{\mathrm{eff}}[\kappa, \lambda]$ up to quadratic order in $\delta \kappa$ and $\delta \lambda$, and neglect all higher orders, we find the so-called Generalized Random Phase Approximation. The latter approach actually gives us also the opportunity to study the density fluctuations and therefore the collective excitations of the gas. We return to this important topic in Section 3.5. Second, it allows for a beautiful way to describe phase transitions. As mentioned previously. in perturbation theory we always find for a homogeneous gas that $G_{\alpha, \alpha^{\prime}}\left(\mathrm{x}, \tau: \mathbf{x}, \tau^{+}\right)=n \delta_{\alpha, \alpha^{\prime}} / 2$ due to the same feature of $G_{0: \alpha, \alpha^{\prime}}\left(\mathbf{x}, \tau ; \mathbf{x}, \tau^{+}\right)$. From a fundamental point of view this is a result of the translational invariance of the gas and of the rotational symmetry in spin space. However, we can imagine that in principle we can also find selfconsistent solutions that do not have this property. We then have a spontaneous breaking of symmetry and therefore a phase transition in our system. For example, if below a certain tomperature $G_{\alpha, \alpha^{\prime}}\left(\mathbf{x}, \tau ; \mathbf{x}, \tau^{+}\right)=n(\mathbf{x}) \delta_{\alpha, \alpha^{\prime}} / 2$, we are dealing with a transition to a charge density wave or (Wigner) crystal. If on the other hand $G_{\alpha, \alpha^{\prime}}\left(\mathrm{x}, \tau ; \mathrm{x}, \tau^{+}\right)=n \delta_{\alpha, \alpha^{\prime}} / 2+\mathbf{m} \cdot \boldsymbol{\sigma}_{\alpha, \alpha^{\prime}}$ the gas is in a ferromagnetic phase. For a spin-density wave we even have that $G_{\alpha, \alpha^{\prime}}\left(\mathbf{x}, \tau: \mathbf{x}, \tau^{+}\right)=n \delta_{\alpha, \alpha^{\prime}} / 2+\mathbf{m}(\mathbf{x}) \cdot \sigma_{\alpha, \alpha^{\prime}}$. In all these cases the HubbardStratonovich approach used above leads in a natural way to the appropriate Landau theory of the phase transition. Since the Landau theory is also very useful for the understanding of superfluidity in atomic gases, which is clearly the primary goal of the present course, we use the next section to give a short introduction into this subject.

\subsection{Landau theory of phase transitions}

In this summary of the Landau theory of phase transitions, we restrict ourselves in first instance to the homogeneous case because this makes the discussion more transparent. However, at the end of the section we also briefly mention how the inhomogeneity enters the theory. We have seen, for example in the Hartree Fock theory discussed above, that the Green's function $G_{\alpha, \alpha^{\prime}}\left(\mathbf{x}, \tau ; \mathbf{x}, \tau^{+}\right)=n \delta_{\alpha, \alpha^{\prime}} / 2+\langle\mathbf{s}\rangle \cdot \boldsymbol{\sigma}_{\alpha, \alpha^{\prime}}$ can have a nonzero value of the average spin density $\langle\mathbf{s}\rangle=\left\langle\hat{\psi}_{\alpha}^{\dagger}(\mathbf{x}, \tau) \boldsymbol{\sigma}_{\alpha, \alpha^{\prime}} \hat{\psi}_{\alpha^{\prime}}(\mathbf{x}, \tau)\right\rangle / 2$, which is usually called the magnetization $\mathbf{m}$ in this context. This signals a phase transition to a ferromagnetic phase and the magnetization is called the order parameter of this transition. In the previous section we have also seen how we can, in principle exactly, obtain an expression for the partition function as 
a)

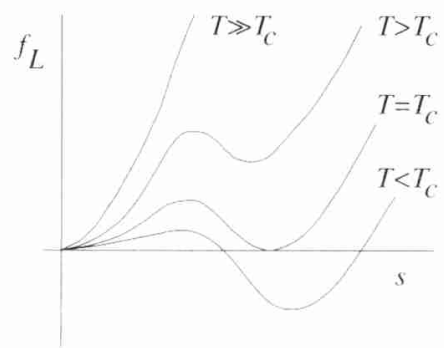

b)

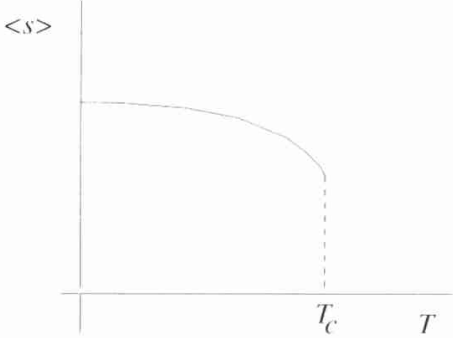

Fig. 10. Qualitative behaviour of a) the Landan free energy and b) the order parameter for a discontinuous phase transition.

a functional integral over the field $\mathbf{s}(\mathbf{x}, \tau)$, i.e..

$$
Z=\int \mathrm{d}[\mathrm{s}] \mathrm{e}^{-S^{\mathrm{fff}}[\mathrm{s}] / h} .
$$

We just have to integrate out the fields $\kappa_{0}$ and $\lambda_{\alpha \cdot \alpha^{\prime}}$. For space and time independent values of the magnetization s the action $S^{\text {eff }}[\mathrm{s}]$ is $h \beta V f_{\mathrm{L}}(\mathbf{s})$, where $f_{\mathrm{L}}(\mathrm{s})$ is the Landau free-energy density and $V$ is the total volume of system. Because of the symmetry of $S\left[\phi^{*}, \phi\right]$ under rotations of the spin, this free-energy density must also be rotationally invariant and can therefore only depend on the magnitude of $s$, which we denote by $s$. If a phase transition occurs the behaviour of $f_{\mathrm{L}}(s)$ can essentially fall only into two categories.

At temperatures very high compared to the critical temperature $T_{\mathrm{c}}$, the system is fully disorded and the free energy $f_{\mathrm{L}}(s)$ must have a single minimum at $s=0$ to make sure that the order parameter $\langle s\rangle$ is zero. Bringing the temperature closer to $T_{\mathrm{c}}$, however, the free energy can develop a second local minimum. As long as the free energy in this second local minimum is higher than the minimum at $s=0$, the equilibrium value of $\langle s\rangle$ will still be zero and no phase transition has occured. Lowering the temperature further. the value of the free energy in the second minimum decrease until, precisely at the critical temperature $T_{c}$, it is equal to the free energy at $s=0$. For temperatures below this critical one the second minimum has actually become the global minimum of the free energy, which implies that $\langle s\rangle \neq 0$ and we are in the ordered phase. In this scenario the order parameter has always a discontinuity at the critical temperature. As a result, this corresponds to a discontinuous, or first-order, phase transition. The behaviour of the free energy and the order parameter is illustrated in Figure 10. This should be compared with the behaviour of the free energy and the order parameter for a continuous, i.e., second or higher order, phase transition, which is quite different and depicted in Figure 11. Now the Landau free energy $f_{\mathrm{L}}(s)$ has always a single minimum, which for temperatures above $T_{c}$ is at $s=0$ but 
a)

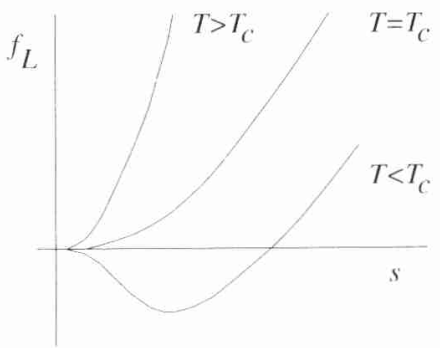

b) $\langle s>$

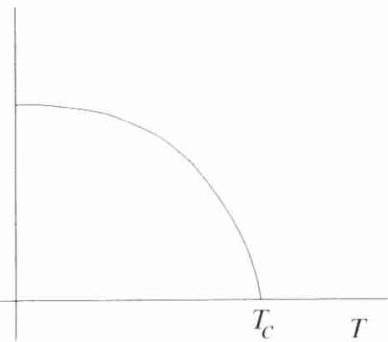

Fig. 11. Qualitative behaviour of a) the Landau free energy and b) the order parameter for a continuous phase transition.

at temperatures below the critical temperature shifts to a nonzero value of s. In particular, the order parameter thus shows no discontinuity at $T_{c}$.

In the case of a second-order phase transition, we conclude that near the critical temperature $\langle s\rangle$ is very small. As a result we can then expand the Landau free-energy density in powers of $s$. Because the free energy must also be symmetric under $s \rightarrow-s$, we have

$$
f_{\mathrm{L}}(\mathbf{s})=\alpha(T)|\mathbf{s}|^{2}+\frac{\beta}{2}|\mathbf{s}|^{4} .
$$

with $\beta>0$ and $\alpha(T)=\alpha_{0}\left(T / T_{\mathrm{c}}-1\right)$. Thus if $T>T_{\mathrm{c}}$ we have $\alpha(T)>0$ and $\langle s\rangle=0$. But for $T<T_{c}, \alpha(T)$ becomes negative and we have

$$
\langle s\rangle=\sqrt{\frac{\alpha_{0}}{\beta}\left(1-\frac{T}{T_{\mathrm{c}}}\right)} .
$$

Note that the free-energy density in this minimum is $-\alpha_{0}^{2}\left(1-T / T_{\mathrm{c}}\right)^{2} / 2 \beta<0$ and has a discontinuity in its second derivative with respect to the temperature. Historically, this is the reason why the corresponding phase transition. was named to be of second order.

In fact, the Landau theory of second order phase transitions is slightly more involved, because it also considers slow spatial fluctuations in s. Since a uniform rotation of $\mathrm{s}$ costs no energy, we must have that the free energy is

$$
F_{\mathrm{L}}[\mathbf{s}]=\int \mathrm{d} \mathbf{x}\left(\gamma(\boldsymbol{\nabla} \mathbf{s}(\mathbf{x}))^{2}+\alpha(T)|\mathbf{s}(\mathbf{x})|^{2}+\frac{\beta}{2}|\mathbf{s}(\mathbf{x})|^{4}\right)
$$

and $Z \simeq \int \mathrm{d}[\mathrm{s}] \mathrm{e}^{-\beta F_{\mathrm{L}}[\mathrm{s}]}$. Landau theory now esentially corresponds to minimizing $F_{\mathrm{L}}[\mathrm{s}]$ and taking only quadratic fluctuations into account. Taking also higher order fluctuations into account turns out to be very difficult. It requires Renormalization Group methods, which we are not going to 
treat here and that can be found in the literature [18]. The cffects of these fluctuation corrections to Landau theory are generally known as critical phenomena. Interestingly, they do not occur in first-order phase transitions. More importantly for our purposes is, however, that for atomic gases the fluctuations are only important in a small temperature interval around the critical temperature. For many applications it is, therefore, possible to neglect them. What usually camnot be negected is the effect of the inhomogeneity of the gas. In the context of Landau theory that implies that the coefficients $\alpha(T), \beta$ and $\gamma$ in equation (2.111) become also dependend on the spatial position in the trapping potential. We will see several examples of this feature in the following.

\subsection{Superfluidity and superconductivity}

We finally want to consider two important second-order phase transitions, that are purely due to quantum effects and can be conveniently treated with the methods that we have developed sofar. Moreover, they occur very often in nature, for example in metals, in liquid helium and recently of course also in atomic gases of rubidium [10], lithium [11], sodium [12] and hydrogen [23].

\subsubsection{Superfluidity}

Let us first consider the last case, which is associated with a gas of spin-less bosons. For the low tempcratures of interest the action is

$$
\begin{aligned}
S\left[\phi^{*}, \phi\right]= & \int_{0}^{\hbar \beta} \mathrm{d} \tau \int \mathrm{d} \mathbf{x} \phi^{*}(\mathrm{x}, \tau)\left\{\hbar \frac{\partial}{\partial \tau}-\frac{\hbar^{2} \nabla^{2}}{2 m}+V^{\mathrm{ex}}(\mathrm{x})-\mu\right\} \phi(\mathrm{x}, \tau) \\
& +\frac{1}{2} \int_{0}^{h \beta} \mathrm{d} \tau \int \mathrm{dx} V_{0} \phi^{*}(\mathrm{x}, \tau) \phi^{*}(\mathrm{x}, \tau) \phi(\mathrm{x}, \tau) \phi(\mathrm{x}, \tau) .
\end{aligned}
$$

where $V\left(\mathbf{x}-\mathbf{x}^{\prime}\right)$ is taken to be equal to $V_{0} \delta\left(\mathbf{x}-\mathbf{x}^{\prime}\right)$. The justification for this simplificaties is, roughly speaking, that the thermal de Broglie wavelength $\Lambda_{\mathrm{th}}=\left(2 \pi h^{2} / m k_{B} T\right)^{1 / 2}$ of the atoms is much larger than the range of the interatomic interaction. In this system the phase transition of interest is Bose-Einstein condensation. The associated order parameter is $\langle\phi(\mathbf{x}, \tau)\rangle$, since for time independent $\phi(\mathrm{x}, \tau)$ the above action has presicely the form of a Landau theory with a "free-energy" of

$$
\begin{aligned}
& F_{\mathrm{L}}\left[\phi^{*}, \phi\right] \\
& \quad=\int \mathrm{d} \mathbf{x}\left(\frac{\hbar^{2}}{2 m}|\nabla \phi(\mathrm{x})|^{2}+\left(V^{\mathrm{ex}}(\mathrm{x})-\mu\right)|\phi(\mathrm{x})|^{2}+\frac{V_{0}}{2}|\phi(\mathrm{x})|^{4}\right) .
\end{aligned}
$$

We conclude that in lowest order the critical temperature is determined by $\mu\left(T_{c}\right)=\epsilon_{\mathbf{0}}$, because then the configurations $\phi(\mathbf{x}) \propto \chi_{\mathbf{0}}(\mathbf{x})$ precisely make 
a vanishing contribution to the quadratic part of the "free energy". This condition makes sense, because it is exactly the condition that we have a Bose-Einstein condensation in the ideal case: In the ideal Bose gas the number of particles in the one-particle ground state is $N_{0}=1 /\left(\mathrm{e}^{\beta\left(\epsilon_{0}-\mu\right)}-1\right)$, which indeed diverges for $\mu=\epsilon_{0}$.

To determine the corrections to this result we now explicitly substitute $\phi(\mathbf{x}, \tau)=\phi_{\mathbf{0}}(\mathrm{x})+\phi^{\prime}(\mathrm{x}, \tau)$ into our functional integral. It is for lateron important to realize that to consistently define the fluctuations $\phi^{\prime}(\mathbf{x}, \tau)$ in this manner, we also have to require that

$$
\int \mathrm{dx} \phi_{0}^{*}(\mathrm{x}) \phi^{\prime}(\mathrm{x}, \tau)+\int \mathrm{dx} \phi_{0}(\mathrm{x}) \phi^{\prime *}(\mathrm{x}, \tau)=0 .
$$

The physical reason behind this condition is that $\phi^{\prime}(\mathrm{x}, \tau)$ should contain all the configurations that are orthogonal to $\phi_{\mathbf{0}}(\mathrm{x})$. In principle, therefore, it should also contain configurations that in effect only multiply $\phi_{\mathbf{0}}(\mathbf{x})$ by a global phase. Such fluctuations lead to the phenomena of phase "diffusion" and are discussed in Section 3.2. In full detail we find after the above substitution that

$$
S\left[\phi^{\prime *}, \phi^{\prime}\right]=\hbar \beta F_{\mathrm{L}}\left[\phi_{\mathbf{0}}^{*}, \phi_{\mathbf{0}}\right]+S_{0}\left[\phi^{\prime *}, \phi^{\prime}\right]+S_{\mathrm{int}}\left[\phi^{\prime *}, \phi^{\prime}\right]
$$

where the linear and quadratic terms are given by

$$
\begin{aligned}
S_{0}\left[\phi^{\prime *}, \phi^{\prime}\right] & \int_{0}^{\hbar \beta} \mathrm{d} \tau \int \mathrm{d} \mathbf{x} \phi^{\prime *}(\mathbf{x}, \tau)\left\{-\frac{\hbar^{2} \nabla^{2}}{2 m}+V^{\mathrm{ex}}(\mathbf{x})-\mu+V_{\mathbf{0}}\left|\phi_{\mathbf{0}}(\mathbf{x})\right|^{2}\right\} \phi_{\mathbf{0}}(\mathbf{x}) \\
& +\int_{0}^{\hbar \beta} \mathrm{d} \tau \int \mathrm{d} \mathbf{x} \phi^{\prime}(\mathbf{x}, \tau)\left\{-\frac{\hbar^{2} \nabla^{2}}{2 m}+V^{\mathrm{ex}}(\mathbf{x})-\mu+V_{\mathbf{0}}\left|\phi_{\mathbf{0}}(\mathbf{x})\right|^{2}\right\} \phi_{\mathbf{0}}^{*}(\mathbf{x}) \\
& +\int_{0}^{\hbar \beta} \mathrm{d} \tau \int \mathrm{d} \mathbf{x} \phi^{\prime *}(\mathbf{x}, \tau) \\
& \times\left\{\hbar \frac{\partial}{\partial \tau}-\frac{\hbar^{2} \nabla^{2}}{2 m}+V^{\mathbf{e x}}(\mathbf{x})-\mu+2 V_{\mathbf{0}}\left|\phi_{\mathbf{0}}(\mathbf{x})\right|^{2}\right\} \phi^{\prime}(\mathbf{x}, \tau) \\
& +\frac{1}{2} \int_{0}^{\hbar \beta} \mathrm{d} \tau \int \mathrm{d} \mathbf{x} V_{\mathbf{0}}\left(\phi_{\mathbf{0}}(\mathbf{x})\right)^{2} \phi^{\prime *}(\mathbf{x}, \tau) \phi^{\prime *}(\mathbf{x}, \tau) \\
& +\frac{1}{2} \int_{0}^{\hbar \beta} \mathrm{d} \tau \int \mathrm{d} \mathbf{x} V_{\mathbf{0}}\left(\phi_{\mathbf{0}}^{*}(\mathbf{x})\right)^{2} \phi^{\prime}(\mathbf{x}, \tau) \phi^{\prime}(\mathbf{x}, \tau),
\end{aligned}
$$


and the cubic and quartic terms by

$$
\begin{aligned}
S_{\text {int }}\left[\phi^{\prime *}, \phi^{\prime}\right]= & \int_{0}^{\hbar \beta} \mathrm{d} \tau \int \mathrm{d} \mathbf{x} V_{\mathbf{0}} \phi_{0}(\mathbf{x}) \phi^{\prime *}(\mathbf{x}, \tau) \phi^{\prime *}(\mathbf{x}, \tau) \phi^{\prime}(\mathbf{x}, \tau) \\
& +\int_{0}^{\hbar \beta} \mathrm{d} \tau \int \mathrm{d} \mathbf{x} V_{\mathbf{0}} \phi_{0}^{*}(\mathbf{x}) \phi^{\prime *}(\mathbf{x}, \tau) \phi^{\prime}(\mathbf{x}, \tau) \phi^{\prime}(\mathbf{x}, \tau) \\
& +\frac{1}{2} \int_{0}^{\hbar \beta} \mathrm{d} \tau \int \mathrm{d} \mathbf{x} V_{0} \phi^{\prime *}(\mathbf{x}, \tau) \phi^{\prime *}(\mathbf{x}, \tau) \phi^{\prime}(\mathbf{x}, \tau) \phi^{\prime}(\mathbf{x}, \tau),
\end{aligned}
$$

respectively.

In the Bogoliubov approximation we neglect the last three interaction terms [24]. Furthermore, to make sure that $\langle\phi(\mathbf{x}, \tau)\rangle=\phi_{\mathbf{0}}(\mathbf{x})$ or $\left\langle\phi^{\prime}(\mathbf{x}, \tau)\right\rangle=$ 0 , we need to require that the terms linear in $\phi^{\prime}$ and $\phi^{\prime *}$ drop out of the action $S_{0}\left[\phi^{\prime *}, \phi^{\prime}\right]$. Clearly, this implies that

$$
\left(-\frac{\hbar^{2} \nabla^{2}}{2 m}+V^{\mathrm{ex}}(\mathrm{x})+V_{\mathbf{0}}\left|\phi_{\mathbf{0}}(\mathrm{x})\right|^{2}\right) \phi_{\mathbf{0}}(\mathrm{x})=\mu \phi_{\mathbf{0}}(\mathrm{x}) .
$$

which is the same result as obtained from minimizing the Landan "freeenergy" $F_{\mathrm{L}}\left[\phi^{*}, \phi\right]$. In the context of trapped atomic gases, this equation is known as the Gross-Pitaevskii equation [25]. It determines the macroscopic wave function of the condensate. The reason for calling $\phi_{0}(\mathrm{x})$ the macroscopic wave function follows from the fact that the total density of the gas now obeys

$$
n(\mathrm{x})=\left\langle\phi(\mathrm{x}, \tau) \phi^{*}\left(\mathbf{x}, \tau^{+}\right)\right\rangle=\left|\phi_{0}(\mathrm{x})\right|^{2}+\left\langle\phi^{\prime}(\mathrm{x}, \tau) \phi^{\prime *}\left(\mathrm{x}, \tau^{+}\right)\right\rangle .
$$

The total number of condensate atoms thus equals $N_{\mathbf{0}}=\int \mathrm{dx}\left|\phi_{\mathbf{0}}(\mathbf{x})\right|^{2}$. As equation (2.119) shows, it is in general always smaller that the total number of atoms in the gas due to the effect of the fluctuations. Note that in our present formulation the average $\left\langle\phi^{\prime}(\mathrm{x}, \tau) \phi^{\prime *}\left(\mathrm{x}, \tau^{+}\right)\right\rangle$physically describes not only the depletion of the condensate due to the usual thermal fluctuations known from the ideal Bose gas, but also due to the interactions, i.e., purely due to quantum fluctuations.

Assuming that we have solved for the Gross-Pitaevskii equation, the fluctuation corrections are in the Bogoliubov theory determined by a quadratic action of the form

$$
\begin{aligned}
& S_{B}\left[\phi^{\prime *}, \phi^{\prime}\right]= \\
& \quad-\frac{\hbar}{2} \int_{0}^{\hbar \beta} \mathrm{d} \tau \int \mathrm{d} \mathbf{x}\left[\phi^{\prime *}(\mathbf{x}, \tau), \phi^{\prime}(\mathbf{x}, \tau)\right] \cdot \mathbf{G}^{-1} \cdot\left[\begin{array}{c}
\phi^{\prime}(\mathbf{x}, \tau) \\
\phi^{\prime *}(\mathbf{x}, \tau)
\end{array}\right],
\end{aligned}
$$

where the associated Green's function $\mathrm{G}$ has now a matrix structure because not only the normal average $\left\langle\phi^{\prime}(\mathrm{x}, \tau) \phi^{\prime *}\left(\mathrm{x}^{\prime}, \tau^{\prime}\right)\right\rangle$ but also the anomalous 
average $\left\langle\phi^{\prime}(\mathbf{x}, \tau) \phi^{\prime}\left(\mathbf{x}^{\prime}, \tau^{\prime}\right)\right\rangle$ is now unequal to zero. We thus have that

$$
-\mathbf{G}\left(\mathbf{x}, \tau ; \mathbf{x}^{\prime}, \tau^{\prime}\right)=\left\langle\left[\begin{array}{c}
\phi^{\prime}(\mathbf{x}, \tau) \\
\phi^{\prime *}(\mathbf{x}, \tau)
\end{array}\right] \cdot\left[\phi^{\prime *}\left(\mathbf{x}^{\prime}, \tau^{\prime}\right), \phi^{\prime}\left(\mathbf{x}^{\prime}, \tau^{\prime}\right)\right]\right\rangle .
$$

From equation (2.116) we in fact find that in the Bogoliubov approximation

$$
\begin{aligned}
\mathbf{G}^{-1}\left(\mathbf{x}, \tau ; \mathbf{x}^{\prime}, \tau^{\prime}\right)= & \mathbf{G}_{0}^{-1}\left(\mathbf{x}, \tau ; \mathbf{x}^{\prime}, \tau^{\prime}\right) \\
& -\frac{1}{\hbar}\left[\begin{array}{cc}
2 V_{\mathbf{0}}\left|\phi_{\mathbf{0}}(\mathbf{x})\right|^{2} & V_{\mathbf{0}}\left(\phi_{\mathbf{0}}(\mathbf{x})\right)^{2} \\
V_{\mathbf{0}}\left(\phi_{\mathbf{0}}^{*}(\mathbf{x})\right)^{2} & 2 V_{0}\left|\phi_{0}(\mathbf{x})\right|^{2}
\end{array}\right] \delta\left(\mathbf{x}-\mathbf{x}^{\prime}\right) \delta\left(\tau-\tau^{\prime}\right) .
\end{aligned}
$$

with the noninteracting Green's function $\mathbf{G}_{0}$ defined by

$$
\mathbf{G}_{0}^{-1}\left(\mathbf{x}, \tau ; \mathbf{x}^{\prime}, \tau^{\prime}\right)=\left[\begin{array}{cc}
G_{0}^{-1}\left(\mathbf{x}, \tau ; \mathbf{x}^{\prime}, \tau^{\prime}\right) & 0 \\
0 & G_{0}^{-1}\left(\mathbf{x}^{\prime}, \tau^{\prime} ; \mathbf{x}, \tau\right)
\end{array}\right]
$$

and, of course,

$$
G_{0}^{-1}\left(\mathbf{x}, \tau ; \mathbf{x}^{\prime}, \tau^{\prime}\right)=-\frac{1}{\hbar}\left\{\hbar \frac{\partial}{\partial \tau}-\frac{\hbar^{2} \nabla^{2}}{2 m}+V^{\mathrm{ex}}(\mathbf{x})-\mu\right\} \delta\left(\mathbf{x}-\mathbf{x}^{\prime}\right) \delta\left(\tau-\tau^{\prime}\right) .
$$

This is clearly only the lowest order result for the Green's function, because a perturbative treatment of $S_{\text {int }}\left[\phi^{\prime *}, \phi^{\prime}\right]$ leads, in the same way as in Section 2.5, to higher order corrections. In general, the Dyson equation is, however, always of the form

$$
\left[\begin{array}{ll}
G_{11} & G_{12} \\
G_{21} & G_{22}
\end{array}\right]^{-1}=\left[\begin{array}{cc}
G_{0}^{-1} & 0 \\
0 & G_{0}^{-1}
\end{array}\right]-\left[\begin{array}{cc}
\Sigma_{11} & \Sigma_{12} \\
\Sigma_{21} & \Sigma_{22}
\end{array}\right]
$$

The off-diagonal elements are again called anomalous, since they vanish in the normal phase of the gas. Diagrammatically the Dyson equations for $G_{11}$ and $G_{21}$ are shown in Figure 12 .

The selfenergy in the Bogoliubov approximation is

$$
\begin{aligned}
& \hbar \mathbf{\Sigma}\left(\mathbf{x}, \tau ; \mathbf{x}^{\prime}, \tau^{\prime}\right) \\
& \quad=\left[\begin{array}{cc}
2 V_{\mathbf{0}}\left|\phi_{\mathbf{0}}(\mathbf{x})\right|^{2} & V_{\mathbf{0}}\left(\phi_{\mathbf{0}}(\mathrm{x})\right)^{2} \\
V_{\mathbf{0}}\left(\phi_{\mathbf{0}}^{*}(\mathrm{x})\right)^{2} & 2 V_{\mathbf{0}}\left|\phi_{\mathbf{0}}(\mathbf{x})\right|^{2}
\end{array}\right] \delta\left(\mathbf{x}-\mathbf{x}^{\prime}\right) \delta\left(\tau-\tau^{\prime}\right) .
\end{aligned}
$$

Because we have completely negelected the cubic and quartic terms in the action to obtain this selfenergy, the Bogoliubov approach is only valid if the fluctuations are sufficiently small. Physically, this impies that the deplection of the condensate must be small. It can, therefore, not be applied to liquid helium, but is expected to be valid for a weakly-interacting atomic 


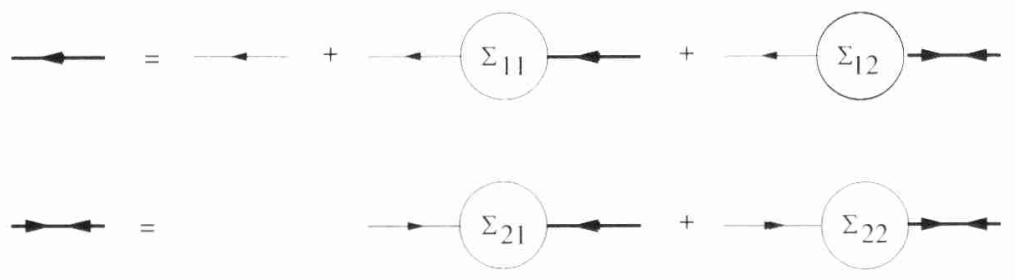

Fig. 12. Exact Dyson equation for the interacting normal and anomalous Green's functions.

gas at such low temperature that it essentially only consists of a condensate. Under these conditions the Bogoliubov theory not only predicts the condensate density profile $n_{0}(\mathrm{x})=\left|\phi_{\mathbf{0}}(\mathrm{x})\right|^{2}$ but also the collective modes of the condensate. Both these predictions have been accurately verificd in recent experiments [26-28]. Theoretically, the eigenfrequencies of the collective modes are again determined by the poles in the one-particle Green's function. To understand more clearly how these can be determined, we first consider a homogeneous Bose gas.

In a box with volume $V=L^{3}$ the one-particle states are most easily characterized by the wavevector $\mathbf{k}=(2 \pi / L) \mathbf{n}$ and equal to $\mathrm{e}^{i \mathbf{k} \cdot \mathbf{x}} / \sqrt{V}$. The oneparticle energies are thus $\epsilon_{\mathbf{k}}=\hbar^{2} \mathbf{k}^{2} / 2 m$. Moreover, the Gross-Pitaevskii equation reduces to $\mu=V_{0}\left|\phi_{0}\right|^{2}$ in that case, because the Landau "frecenergy" is minimized for a macroscopic wave function that is independent of the position in the box. Making use of this fact, equation (2.122) can immediately be solved by a Fourier transformation. The result is

$$
-\hbar \mathbf{G}^{-1}\left(\mathbf{k}, i \omega_{n}\right)=\left[\begin{array}{cc}
-i \hbar \omega_{n}+\epsilon_{\mathbf{k}}+V_{\mathbf{0}}\left|\phi_{\mathbf{0}}\right|^{2} & V_{\mathbf{0}} \phi_{0}^{2} \\
V_{\mathbf{0}} \phi_{\mathbf{0}}^{* 2} & i \hbar \omega_{n}+\epsilon_{\mathbf{k}}+V_{\mathbf{0}}\left|\phi_{\mathbf{0}}\right|^{2}
\end{array}\right] .
$$

Clearly there are poles in $G(\mathbf{k}, \omega)$ if the determinant of the right-hand side is zero or if

$$
\hbar \omega=\hbar \omega_{\mathbf{k}} \equiv \sqrt{\epsilon_{\mathbf{k}}^{2}+2 V_{\mathbf{0}}\left|\phi_{\mathbf{0}}\right|^{2} \epsilon_{\mathbf{k}}}=\sqrt{\epsilon_{\mathbf{k}}^{2}+2 V_{0} n_{\mathbf{0}} \epsilon_{\mathbf{k}}} .
$$

This is the famous Bogoliubov dispersion of the collective excitations. Note that to finish the calculation we still have to obtain the condensate density $n_{0}=\left|\phi_{\mathbf{0}}\right|^{2}$. This is determined by the total density of the gas, which obeys

$$
n=\left|\phi_{0}\right|^{2}-G_{11}\left(\mathbf{x}, \tau ; \mathbf{x}, \tau^{+}\right)=n_{\mathbf{0}}+n^{\prime},
$$

with $n^{\prime}$ the density of the "above" condensate particles. By inverting the right-hand side of equation (2.128), we find that the noncondensate density 
is equal to

$$
\begin{aligned}
n^{\prime} & =\lim _{\eta ! 0} \frac{\hbar}{V \hbar \beta} \sum_{\mathbf{k} \neq 0 . n} \mathrm{e}^{i \omega_{n} \eta} \frac{i \hbar \omega_{n}+\epsilon_{\mathbf{k}}+V_{\mathbf{0}} n_{\mathbf{0}}}{\left(\hbar \omega_{n}\right)^{2}+\left(\hbar \omega_{\mathbf{k}}\right)^{2}} \\
& =\frac{1}{V} \sum_{\mathbf{k} \neq \mathbf{0}}\left(\frac{\epsilon_{\mathbf{k}}+V_{\mathbf{0}} n_{0}}{\hbar \omega_{\mathbf{k}}} \frac{1}{\left.\mathrm{e}^{\beta \hbar \omega_{\mathbf{k}}-1}+\frac{\epsilon_{\mathbf{k}}+V_{0} n_{0}-\hbar \omega_{\mathbf{k}}}{2 \hbar \omega_{\mathrm{k}}}\right) .}\right.
\end{aligned}
$$

For a given density and temporature, the last two equations thus fully determine the condensate density. Note that equation (2.131) explicitly shows that the condensate is indeed depleted both by thermal as well as quantum effects.

The generalization to the inhomogeneous case is straightforward. First we again have to solve the Gross-Pitaevskii equation at a fixed chemical potential. Given the condensate wave function, we can then calculate the collective mode frequencies by finding the poles of $\mathbf{G}$, or equivalently but more conveniently, the zero's of $\mathbf{G}^{-1}$. Clearly, the latter are located at $\hbar \omega=\hbar \omega_{\mathbf{n}}$, whore $\hbar \omega_{\mathbf{n}}$ is found from the cigenvalue problem

$$
\begin{array}{r}
{\left[\begin{array}{cc}
\hat{K}+2 V_{\mathbf{0}}\left|\phi_{\mathbf{0}}(\mathbf{x})\right|^{2} & V_{\mathbf{0}}\left(\phi_{\mathbf{0}}(\mathbf{x})\right)^{2} \\
V_{\mathbf{0}}\left(\phi_{0}^{*}(\mathbf{x})\right)^{2} & \hat{K}+2 V_{\mathbf{0}}\left|\phi_{\mathbf{0}}(\mathbf{x})\right|^{2}
\end{array}\right] \cdot\left[\begin{array}{c}
u_{\mathbf{n}}(\mathbf{x}) \\
v_{\mathbf{n}}(\mathbf{x})
\end{array}\right]} \\
=\hbar \omega_{\mathbf{n}}\left[\begin{array}{cc}
1 & 0 \\
0 & -1
\end{array}\right] \cdot\left[\begin{array}{l}
u_{\mathbf{n}}(\mathbf{x}) \\
v_{\mathbf{n}}(\mathbf{x})
\end{array}\right]
\end{array}
$$

and we introduced the operator $\hat{K}=-\hbar^{2} \nabla^{2} / 2 m+V^{\mathrm{ex}}(\mathrm{x})-\mu$. This is de Bogoliubov-de Gemnes equation that has recently been applied with great succes to the collective modes of a Bose condensed rubidium and sodium gas $[29,30]$. Note that a special solution with $\hbar \omega_{0}=0$ is given by $\left[u_{0}(\mathrm{x}), v_{0}(\mathrm{x})\right]=\left[\phi_{\mathbf{0}}(\mathrm{x}),-\phi_{\mathbf{0}}^{*}(\mathrm{x})\right]$. As a result we can, by making use of the fact that the left-hand side of the Bogoliubov-de Gennes equation involves a hermitian operator, easily proof that all the solutions obey

$$
\int \mathrm{d} \mathbf{x} \phi_{\mathbf{0}}^{*}(\mathbf{x}) u_{\mathbf{n}}(\mathbf{x})+\int \mathrm{d} \mathbf{x} \phi_{\mathbf{0}}(\mathbf{x}) v_{\mathbf{n}}(\mathbf{x})=0,
$$

as required by the condition in equation (2.114). Moreover, we can similary show that the solutions with $\hbar \omega_{\mathbf{n}}>0$ can always be normalized as [31]

$$
\int \mathrm{dx}\left(\left|u_{\mathbf{n}}(\mathrm{x})\right|^{2}-\left|v_{\mathbf{n}}(\mathrm{x})\right|^{2}\right)=1 .
$$

Physically, the zero frequency solution $\mathbf{n}=\mathbf{0}$ describes the dynamics of the global phase of the condensate [32]. Because of the so-called $U(1)$ symmetry of the action, i.e., its invariance under the simultaneous phase changes $\phi(\mathbf{x}, \tau) \rightarrow \mathrm{e}^{i \vartheta} \phi(\mathbf{x}, \tau)$ and $\phi^{*}(\mathbf{x}, \tau) \rightarrow \mathrm{e}^{-i \vartheta} \phi^{*}(\mathbf{x}, \tau)$, this solution is essentially of no importance to the thermodynamic properties of a macroscopic 
a)

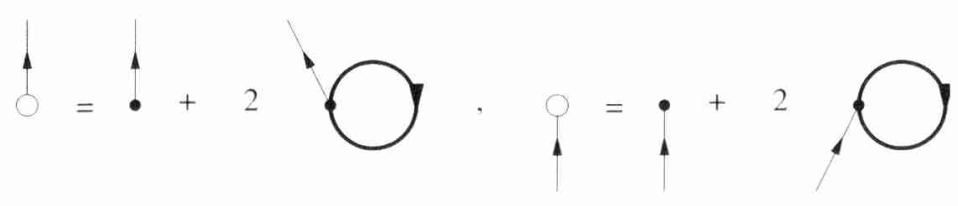

b)

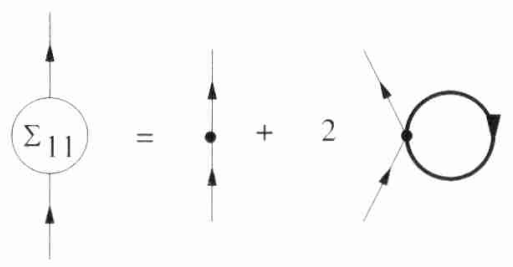

Fig. 13. Hartree-Fock corrections to a) the linear and b) the quadratic interaction terms of the Bogoliubov theory. This represents the Popov theory.

gas sample and is therefore usually neglected. Nevertheless, it has from a fundamental point of view some interesting consequences, as we will see in Section 3.2. Knowing all the eigenstates of $\mathbf{G}^{-1}$ we can then casily perform the inversion and finally again determine the density profile of the noncondensed atoms. Keeping the physical significance of the zero frequency mode in mind, we ultimately find,

$$
n^{\prime}(\mathbf{x})=\sum_{\mathbf{n} \neq \mathbf{0}}\left(\left(\left|u_{\mathbf{n}}(\mathbf{x})\right|^{2}+\left|v_{\mathbf{n}}(\mathbf{x})\right|^{2}\right) \frac{1}{\mathrm{e}^{\beta \hbar \omega_{\mathbf{n}}}-1}+\left|v_{\mathbf{n}}(\mathbf{x})\right|^{2}\right),
$$

which may be compared with equation (2.131).

At temperatures near absolute zero, we have as a good approximation that $n^{\prime}(\mathbf{x})=0$ and the Bogoliubov theory applies. However at nonzero temperatures we thermally excite particles and $n^{\prime}(\mathrm{x})$ becomes nonzero. If we treat the effect of the noncondensate part of the gas in the Hartree Fock approximation, we find that $S_{\text {int }}\left[\phi^{\prime *}, \phi^{\prime}\right]$ on average adds

$$
\begin{aligned}
S_{\text {int }}^{\mathrm{HF}}\left[\phi^{\prime *}, \phi^{\prime}\right]= & 2 \int_{0}^{\hbar \beta} \mathrm{d} \tau \int \mathrm{d} \mathbf{x} V_{0} n^{\prime}(\mathrm{x}) \phi^{\prime *}(\mathrm{x}, \tau) \phi_{\mathbf{0}}(\mathrm{x}) \\
& +2 \int_{0}^{\hbar \beta} \mathrm{d} \tau \int \mathrm{d} \mathbf{x} V_{0} n^{\prime}(\mathbf{x}) \phi^{\prime}(\mathbf{x}, \tau) \phi_{\mathbf{0}}^{*}(\mathbf{x}) \\
& +2 \int_{0}^{\hbar \beta} \mathrm{d} \tau \int \mathrm{d} \mathbf{x} V_{0} n^{\prime}(\mathbf{x}) \phi^{\prime *}(\mathbf{x}, \tau) \phi^{\prime}(\mathbf{x}, \tau),
\end{aligned}
$$

to the action $S_{0}\left[\phi^{\prime *}, \phi^{\prime}\right]$. In Figure 13 we indicate how this can be understood diagramatically. Performing the same analysis as before, we conclude that 
the Gross-Pitaevskii equation is modified to

$$
\left(-\frac{\hbar^{2} \nabla^{2}}{2 m}+V^{\mathrm{ex}}(\mathrm{x})+2 V_{0} n^{\prime}(\mathrm{x})+V_{0}\left|\phi_{0}(\mathrm{x})\right|^{2}\right) \phi_{0}(\mathrm{x})=\mu \phi_{0}(\mathrm{x}),
$$

and the normal selfenergies are changed into $2 V_{0}\left|\phi_{0}(\mathbf{x})\right|^{2}+2 V_{0} n^{\prime}(\mathbf{x})=$ $2 V_{0} n(\mathbf{x})$. The Bogoliubov-de Gennes equation for the elementary excitations is, therefore, now given by

$$
\begin{aligned}
& {\left[\begin{array}{cc}
\hat{K}+2 V_{\mathbf{0}} n(\mathbf{x}) & V_{\mathbf{0}}\left(\phi_{0}(\mathbf{x})\right)^{2} \\
V_{\mathbf{0}}\left(\phi_{\mathbf{0}}^{*}(\mathbf{x})\right)^{2} & \hat{K}+2 V_{\mathbf{0}} n(\mathbf{x})
\end{array}\right] \cdot\left[\begin{array}{c}
u_{\mathbf{n}}(\mathbf{x}) \\
v_{\mathbf{n}}(\mathbf{x})
\end{array}\right]} \\
& =\hbar \omega_{\mathbf{n}}\left[\begin{array}{cc}
1 & 0 \\
0 & -1
\end{array}\right] \cdot\left[\begin{array}{l}
u_{\mathbf{n}}(\mathbf{x}) \\
v_{\mathbf{n}}(\mathbf{x})
\end{array}\right] \text {. }
\end{aligned}
$$

These last two equations in combination with equation (2.135) are known as the Popov theory in the recent literature [33]. It is much studied at present in the context of Bose-Einstein condensation in atomic gases, and has been applied with succes to the equilibrium density profile of the gas below the critical temperature [34]. It has also been used to determine the collective mode frequencies of the gas at nonzero temperatures, however, with much less success $[35,36]$. The reason for the failure of the Popov theory in this case is that the Bogoliubov-de Gennes equation in equation (2.138) describes physically only the motion of the condensate in the presence of a static noncondensed cloud and not the dynamics of the noncondensed cloud itself. How that can be achieved is discussed lateron in Section 3.5, when we have obtained a better understanding of the nonequilibrium theory. Now wo briefly want to make a connection to the interaction parameter $V_{0}$ used in the Bogoliubov and Popov theories and the specific two-body scattering properties of the atomic gas of interest in a particular experiment.

\subsubsection{Some atomic physics}

In the previous section we mentioned that the Bogoliubov theory, and to a certain extent also the Popov theory, agree very well with experiment. It is clear, however, that to apply these theories to an actual experiment we need to know the interaction parameter $V_{0}$. In the case of atomic gases it is indeed possible to perform an ab initio calculation of this quantity, something which for instance cannot be done for liquid helium. The reason why $V_{0}$ can be determined for atomic gases, is that under the experimental conditions of interest the densities are always so low that we only need to consider all two-body processes taking place in the gas and we can neglect three-body and higher-body processes. This implies physically that we only have to calculate and add the quantum mechanical amplitudes for two atoms to scatter of each other an arbitrary number of times. Diagrammatically 

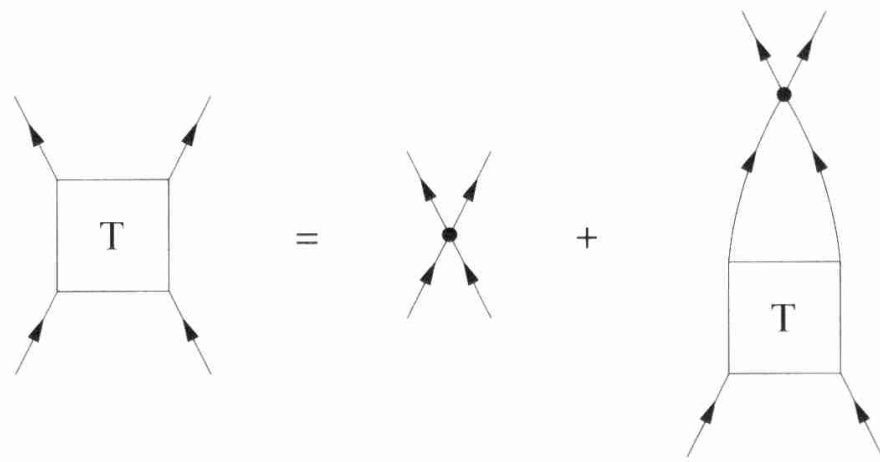

Fig. 14. T-matrix equation for the effective interatomic interaction.

the procedure can essentially be summarized by the T-matrix equation in Figure 14, because by iteration of this equation we casily see that we are indeed summing all two-body interacion processes.

Denoting the total momentum of the two incoming particles by $\hbar \mathbf{K}$ and the sum of the two Matsubara frequencies by $\Omega_{n}$, the T-matrix equation in Figure 14 can, with our knowledge of how to perform the Matsubara sum in the right-hand side, easily be shown to be mathematically equivalent to

$$
\begin{aligned}
T\left(\mathbf{K}, i \Omega_{n}\right)= & V_{\mathbf{0}}+\frac{V_{0}}{V} \sum_{\mathbf{k}} \frac{1}{i \hbar \Omega_{n}-\epsilon_{\mathbf{K} / 2+\mathbf{k}}-\epsilon_{\mathbf{K} / 2-\mathbf{k}}+2 \mu} \\
& \times\left(1+\frac{1}{\mathrm{e}^{\beta\left(\epsilon_{\mathbf{K} / 2+\mathbf{k}}-\mu\right)}-1}+\frac{1}{\mathrm{e}^{\beta\left(\epsilon_{\mathbf{K} / 2-\mathbf{k}}-\mu\right)}-1}\right) T\left(\mathbf{K}, i \Omega_{n}\right) .
\end{aligned}
$$

It is immediately solved by

$$
\begin{aligned}
\frac{1}{T\left(\mathbf{K}, i \Omega_{n}\right)}= & \frac{1}{V_{\mathbf{0}}}-\frac{1}{V} \sum_{\mathbf{k}} \frac{1}{i \hbar \Omega_{n}-\epsilon_{\mathbf{K} / 2+\mathbf{k}}-\epsilon_{\mathbf{K} / 2-\mathbf{k}}+2 \mu} \\
& \times\left(1+\frac{1}{\mathrm{e}^{\beta\left(\epsilon_{\mathbf{K} / 2+\mathbf{k}}-\mu\right)}-1}+\frac{1}{\mathrm{e}^{\beta\left(\epsilon_{\mathbf{K} / 2-\mathbf{k}}-\mu\right)}-1}\right) .
\end{aligned}
$$

Note that this is a somewhat formal result, because the momentum sum in the right-hand side has an ultra-violet divergence, which from a fieldtheoretical point of view requires a renormalization procedure.

The physical reason for the divergence is that we have used the approximate potential $V_{\mathbf{0}} \delta\left(\mathbf{x}-\mathbf{x}^{\prime}\right)$ instead of the actual interactomic potential $V\left(\mathbf{x}-\mathbf{x}^{\prime}\right)$. The argument for using the $\delta$-function approximation was that the thermal de Broglie wavelength of the atoms is for the ultra-low temperatures of interest always much larger than the typical range of the interaction. We now see that this argument is not fully correct, because if we calculate 
corrections in perturbation theory, we have to deal with momentum sums which are not always restricted to momenta of order $\hbar / \Lambda_{\text {th }}$ and are therefore sensitive to the precise details of the interaction potential.

To cure this disease we note that if we put in equation (2.139) the Bose occupation numbers equal to zero, we precisely get the T-matrix or Lippmann-Schwinger equation [37] for two atoms interacting with the potential $V_{0} \delta\left(\mathrm{x}-\mathrm{x}^{\prime}\right)$. Furthermore, if the atoms interact with the potential $V\left(\mathrm{x}-\mathrm{x}^{\prime}\right)$ the solution of the corresponding Lippmann-Schwinger equation is known from elementary scattering theory to be equal to $4 \pi a \hbar^{2} / m$ for small incoming momenta and energies, where $a$ is the $s$-wave scattering length. We thus conclude that we must interpret

$$
\frac{1}{V_{\mathbf{0}}}-\frac{1}{V} \sum_{\mathbf{k}} \frac{1}{i \hbar \Omega_{n}-\epsilon_{\mathbf{K} / 2+\mathbf{k}}-\epsilon_{\mathbf{K} / 2-\mathbf{k}}+2 \mu}
$$

as being equal to $m / 4 \pi a \hbar^{2}$ and, therefore, that the desired T-matrix in principle obeys

$$
\begin{aligned}
\frac{1}{T\left(\mathbf{K}, i \Omega_{n}\right)}= & \frac{m}{4 \pi a \hbar^{2}}-\frac{1}{V} \sum_{\mathbf{k}} \frac{1}{i \hbar \Omega_{n}-\epsilon_{\mathbf{K} / 2+\mathbf{k}}-\epsilon_{\mathbf{K} / 2-\mathbf{k}}+2 \mu} \\
& \times\left(\frac{1}{\mathrm{e}^{\beta\left(\epsilon_{\mathbf{K} / 2+\mathbf{k}}-\mu\right)}-1}+\frac{1}{\mathrm{e}^{\beta\left(\epsilon_{\mathbf{K} / 2-\mathbf{k}}-\mu\right)}-1}\right) .
\end{aligned}
$$

To obtain an accurate theory for a trapped atomic Bose gas that includes the effect of all two-body processes, we should now use instead of $V_{0}$ the above T-matrix in the Bogoliubov or Popov theories. Of course, in the normal phase the same is true for the Hartree Fock theory. If the temperature is not too close to the critical temperature for Bose-Einstein condensation, it turns out that $T\left(\mathbf{K}, i \Omega_{n}\right) \simeq 4 \pi a \hbar^{2} / m$ and we arrive at the conclusion that for an application to realistic atomic gases we must replace everywhere in Section 2.8.1 the interaction parameter $V_{0}$ by $4 \pi a \hbar^{2} / m$. For temperatures close to the critical temperature, this is however no longer true and the Bose distribution functions have a nonnegligible effect on the T-matrix $[38,39]$. The theory that, by using "dressed" one-particle propagators in Figure 14, selfconsistently includes these effects of the medium on the scattering properties of the atoms is known as the many-body T-matrix theory. It results in an effective interaction that, to avoid confusion, is denoted by $T^{(+)}(\mathbf{K}, \Omega)$ in Section 3.4. As we will see then, it plays a crucial role if we want to arrive at a consistent discription of condensate formation in an atomic Bose gas.

\subsubsection{Superconductivity}

Finally, we want to briefly discuss the Bardeen-Cooper-Schrieffer or BCS theory of superconductivity [40], which has received considerable attention 
recently in connection with ongoing experiments with the fermionic isotope of lithium. The reasons for this will become clear shortly. We are in that case dealing with effective spin $1 / 2$ fermions and the action is taken to be

$$
\begin{aligned}
S\left[\phi^{*}, \phi\right]= & \sum_{\alpha=\uparrow, \downarrow} \int_{0}^{\hbar \beta} \mathrm{d} \tau \int \mathrm{d} \mathbf{x} \phi_{\alpha}^{*}(\mathbf{x}, \tau)\left\{\hbar \frac{\partial}{\partial \tau}-\frac{\hbar^{2} \nabla^{2}}{2 m}-\mu\right\} \phi_{\alpha}(\mathbf{x}, \tau) \\
& +\int_{0}^{\hbar \beta} \mathrm{d} \tau \int \mathrm{d} \mathbf{x} V_{\mathbf{0}} \phi_{\uparrow}^{*}(\mathbf{x}, \tau) \phi_{\downarrow}^{*}(\mathbf{x}, \tau) \phi_{\downarrow}(\mathbf{x}, \tau) \phi_{\uparrow}(\mathbf{x}, \tau) .
\end{aligned}
$$

Note that the use of a single chemical potential implies that we only consider the optimal case of an equal density in each hyperfine state. This situation is optimal in the sense that, for mequal densities in each hyperfine state. it is no longer possible to pair up all the atoms in the gas. As a result the critical temperature of the gas drops dramatically, and in general becomes experimentally inaccessible. The effect is to a large extent analogous to putting superfluid ${ }^{3} \mathrm{He}$ in an homogeneous magnetic ficld. Furthermore, we in first instance consider the homogeneous case, because we want to illustrate in this section the local-density approximation to include the effect of the external trapping potential. Physically, this approximation treats the gas as consisting of a large number of independent gases that are in diffusive equilibrium with each other. Such an approach only works if the correlation length of the gas is much smaller than the typical length scale associated with changes of the external potential. Fortumately, this is almost always the case for realistic trapped atomic gases and for that reason the local-density approximation is often used in practice.

The BCS theory is the theory of Bose-Einstein condensation of so-called Cooper pairs. This means that the order parameter is $\left\langle\phi_{1}(\mathbf{x}, \tau) \phi_{\uparrow}(\mathbf{x}, \tau)\right\rangle$, in analogy with the order parameter $\langle\phi(\mathbf{x}, \tau)\rangle$ for the Bose case just discussed. Furthermore, it requires that the interaction paraneter $V_{0}$ is negative, since otherwise the formation of pairs would not be energetically favorable. From now on we assume, therefore, that this is the case. The condensate of Cooper pairs can also be nicely treated with a Hubbard-Stratonovich transformation. We now introduce a complex field $\Delta(\mathrm{x}, \tau)$ and use

$$
\begin{aligned}
\exp \left\{-\frac{1}{\hbar} \int_{0}^{\hbar \beta} \mathrm{d} \tau \int \mathrm{dx} V_{\mathbf{0}} \phi_{\uparrow}^{*}(\mathrm{x}, \tau) \phi_{\downarrow}^{*}(\mathrm{x}, \tau) \phi_{\rfloor}(\mathrm{x}, \tau) \phi_{\uparrow}(\mathrm{x}, \tau)\right\} \\
=\int \mathrm{d}\left[\Delta^{*}\right] \mathrm{d}[\Delta] \exp \left\{\frac { 1 } { \hbar } \int _ { 0 } ^ { \hbar \beta } \mathrm { d } \tau \int \mathrm { dx } \left(\frac{|\Delta(\mathrm{x}, \tau)|^{2}}{V_{0}}\right.\right. \\
\left.\left.\quad+\Delta^{*}(\mathbf{x}, \tau) \phi_{\downarrow}(\mathrm{x}, \tau) \phi_{\uparrow}(\mathbf{x}, \tau)+\phi_{\uparrow}^{*}(\mathrm{x}, \tau) \phi_{\downarrow}^{*}(\mathrm{x}, \tau) \Delta(\mathbf{x}, \tau)\right)\right\}
\end{aligned}
$$


This leads to a partition function with the action

$$
\begin{aligned}
& S\left[\Delta^{*}, \Delta, \phi^{*}, \phi\right]=-\int_{0}^{\hbar \beta} \mathrm{d} \tau \int \mathrm{d} \mathbf{x} \frac{|\Delta(\mathbf{x}, \tau)|^{2}}{V_{0}} \\
& \quad-\frac{\hbar}{2} \int_{0}^{h \beta} \mathrm{d} \tau \int \mathrm{d} \mathbf{x}\left[\phi_{!}^{*}(\mathbf{x}, \tau), \phi_{\uparrow}(\mathbf{x}, \tau)\right] \cdot \mathbf{G}^{-1} \cdot\left[\begin{array}{c}
\phi_{\downarrow}(\mathbf{x}, \tau) \\
\phi_{\uparrow}^{*}(\mathbf{x}, \tau)
\end{array}\right],
\end{aligned}
$$

where

$$
\begin{aligned}
\mathbf{G}^{-1}\left(\mathbf{x}, \tau ; \mathbf{x}^{\prime}, \tau^{\prime}\right)= & \mathbf{G}_{0}^{-1}\left(\mathbf{x}, \tau ; \mathbf{x}^{\prime}, \tau^{\prime}\right) \\
& -\frac{1}{\hbar}\left[\begin{array}{cc}
0 & \Delta(\mathbf{x}, \tau) \\
\Delta^{*}(\mathbf{x}, \tau) & 0
\end{array}\right] \delta\left(\mathbf{x}-\mathbf{x}^{\prime}\right) \delta\left(\tau-\tau^{\prime}\right),
\end{aligned}
$$

and the noninteracting Green's function $\mathbf{G}_{0}$ is defined by

$$
\mathbf{G}_{0}^{-1}\left(\mathbf{x}, \tau ; \mathbf{x}^{\prime}, \tau^{\prime}\right)=\left[\begin{array}{cc}
G_{0}^{-1}\left(\mathbf{x}, \tau ; \mathbf{x}^{\prime}, \tau^{\prime}\right) & 0 \\
0 & -G_{0}^{-1}\left(\mathbf{x}^{\prime}, \tau^{\prime} ; \mathbf{x}, \tau\right)
\end{array}\right]
$$

and

$$
G_{0}^{-1}\left(\mathbf{x}, \tau ; \mathbf{x}^{\prime}, \tau^{\prime}\right)=-\frac{1}{h}\left\{\hbar \frac{\partial}{\partial \tau}-\frac{\hbar^{2} \nabla^{2}}{2 m}-\mu\right\} \delta\left(\mathbf{x}-\mathbf{x}^{\prime}\right) \delta\left(\tau-\tau^{\prime}\right) .
$$

We thus see that the fermionic part has exactly the same matrix structure as in the case of a condensed Bose gas, only the selfenergy is now

$$
\left[\begin{array}{cc}
\Sigma_{11} & \Sigma_{12} \\
\Sigma_{21} & \Sigma_{22}
\end{array}\right]=\frac{1}{\hbar}\left[\begin{array}{cc}
0 & \Delta(\mathbf{x}, \tau) \\
\Delta^{*}(\mathbf{x}, \tau) & 0
\end{array}\right] \delta\left(\mathbf{x}-\mathbf{x}^{\prime}\right) \delta\left(\tau-\tau^{\prime}\right) .
$$

If we again integrate out the fermion fields, we get the effective action

$$
S^{\text {eff }}\left[\Delta^{*}, \Delta\right]=-\int_{0}^{h \beta} \mathrm{d} \tau \int \mathrm{d} \mathbf{x} \frac{|\Delta(\mathbf{x}, \tau)|^{2}}{V_{0}}-\hbar \operatorname{Tr}\left[\ln \left(-\mathbf{G}^{-1}\right)\right],
$$

which we can expand in powers of $\Delta$ by using $\mathbf{G}^{-1}=\mathbf{G}_{0}^{-1}-\mathbf{\Sigma}=\mathbf{G}_{0}^{-1}(1-$ $\left.\mathbf{G}_{0} \mathbf{\Sigma}\right)$ and therefore

$$
-\hbar \operatorname{Tr}\left[\ln \left(-\mathbf{G}^{-1}\right)\right]=-\hbar \operatorname{Tr}\left[\ln \left(-\mathbf{G}_{0}^{-1}\right)\right]+\hbar \sum_{m=1}^{\infty} \frac{1}{m} \operatorname{Tr}\left[\left(\mathbf{G}_{0} \mathbf{\Sigma}\right)^{m}\right] .
$$

Explicite calculation [41] shows that for space and time independent $\Delta$ we obtain a "free-energy" density of the form of the Landau-theory of secondorder phase transitions, i.e.,

$$
f_{\mathrm{L}}(|\Delta|)=\alpha(T)|\Delta|^{2}+N(0) \frac{7 \zeta(3)}{16\left(\pi T_{\mathrm{c}}\right)^{2}}|\Delta|^{4},
$$


with as expected

$$
\begin{aligned}
\alpha(T) & =-\frac{1}{V_{0}}+\frac{1}{V} \sum_{\mathbf{k}} \frac{1}{2\left(\epsilon_{\mathbf{k}}-\mu\right)}\left(1-\frac{2}{\mathrm{e}^{\beta\left(\epsilon_{\mathbf{k}}-\mu\right)}+1}\right) \\
& \equiv N(0) \ln \left(\frac{T}{T_{\mathrm{c}}}\right) \simeq N(0)\left(\frac{T}{T_{\mathrm{c}}}-1\right) .
\end{aligned}
$$

Here $N(0)$ is the density of states of a single spin state at the Formi energy $\epsilon_{\mathrm{F}} \equiv \hbar^{2} k_{\mathrm{F}}^{2} / 2 m$ and the critical temperature is given by

$$
T_{\mathrm{C}}=\frac{8 \mathrm{e}^{\gamma-2}}{\pi} \frac{\epsilon_{\mathrm{F}}}{k_{B}} \exp \left\{-\frac{\pi}{2 k_{\mathrm{F}}|a|}\right\} \text {. }
$$

if we use the same renormalization procedure as in Section 2.8 .2 to eliminate the interaction parameter $V_{\mathbf{0}}$ in favor of the negative s-wave scattering length $a$.

Below $T_{\mathrm{C}}$ we thus have a nonzero average $\langle\Delta(\mathrm{x}, \tau)\rangle \equiv \Delta_{0}$. Using this in the Green's function for the fermions and neglecting fluctations, we find in momentum space that

$$
-\hbar \mathbf{G}^{-1}\left(\mathbf{k}, i \omega_{n}\right)=\left[\begin{array}{cc}
-i \hbar \omega_{n}+\epsilon_{\mathrm{k}}-\mu & \Delta_{0} \\
\Delta_{0}^{*} & -\left(i \hbar \omega_{n}+\epsilon_{\mathrm{k}}-\mu\right)
\end{array}\right]
$$

and therefore poles in $\mathbf{G}(\mathbf{k}, \omega)$ if

$$
\hbar \omega=\hbar \omega_{\mathbf{k}}=\sqrt{\left(\epsilon_{\mathbf{k}}-\mu\right)^{2}+\left|\Delta_{0}\right|^{2}} .
$$

This disperion relation has clearly a gap of magnitude $\left|\Delta_{0}\right|$ at the Fermi surface. As a consequence $\left|\Delta_{0}\right|$ is known as the BCS gap parameter. Note that the critical temperature below which the gap becomes nonzero depends exponentally on the parameter $\pi / 2 k_{\mathrm{F}}|a|$. For typical atomic gases this is a quantity which is much larger than one, and the BCS transition is experimentally inaccesible. The only exception at the moment appears to be ${ }^{6} \mathrm{Li}$, with its anomalously large and negative triplet scattering length of $-2160 a_{0}[42]$. This explains the present interest in spin-polarized atomic lithium.

In view of this exciting possibility, we have recently studied the equilibrium properties of atomic lithium is a harmonic oscillator potential $V^{\text {ex }}(\mathbf{x})=$ $m \omega^{2} \mathrm{x}^{2} / 2$, with a trapping frequency of $\omega / 2 \pi=144 \mathrm{~Hz}$ [43]. To incorporate the effect of the external potential we have, as mentioned above, applied the local-density approximation. The result is shown in Figure 15. Note that the use of the local-density approximation implies that we perform the above outlined homogeneous calculation for each point in space with a chemical potential that is equal to

$$
\mu(\mathbf{x})=\mu-\frac{1}{2} m \omega^{2} \mathbf{x}^{2}-\frac{4 \pi a \hbar^{2}}{m} \frac{n(\mathbf{x})}{2} .
$$


a)

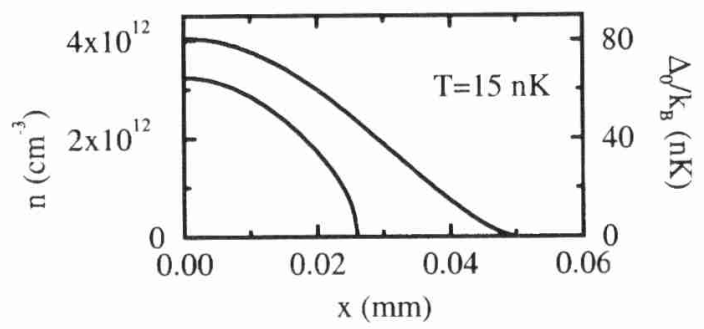

b)

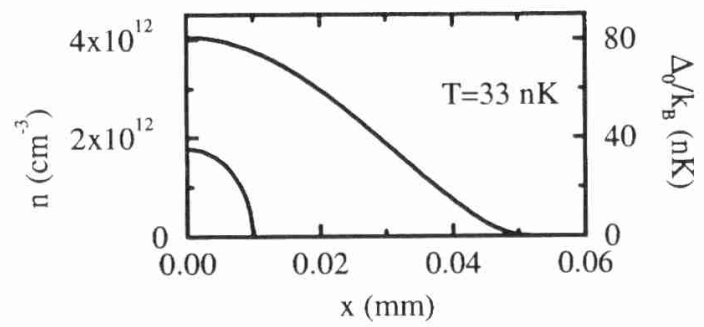

c)

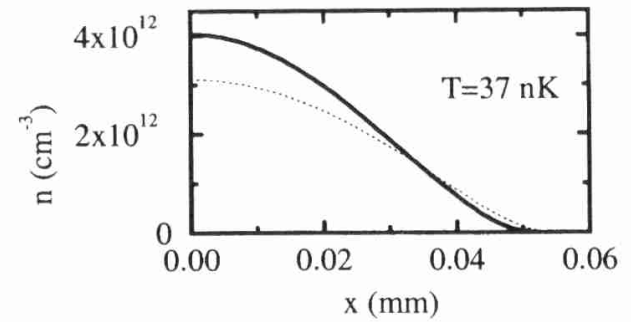

Fig. 15. Density distribution $n(\mathrm{x})$ and energy gap $\Delta_{0}(\mathbf{x})$ for a ${ }^{6} \mathrm{Li}$ atomic gas consisting of $2.865 \times 10^{5}$ atoms in each spin state a) at $T=15 \mathrm{nK}$, b) at $T=33 \mathrm{nK}$, slightly below $T_{c}$, and c) at $T=T_{\mathrm{c}}=37 \mathrm{nK}$. The left scale of each plot refers to the density and the right scale to the energy gap. The dotted line in c) shows the density distribution for an ideal Fermi gas with the same number of particles and at the same temperature.

The third term in the right-hand side represents the mean-field effect of the Hartree contribution to the selfenergy of the fermions. Since our HubbardStratonovich procedure is in principle exact, it is not immediately clear why such a term must be included in the theory. It can, however, be shown that it arises from the fluctuations of the BCS gap parameter. With this remark, we end our development of the equilibrium field theory of trapped atomic gases. We now turn our attention to the nonequilibrium field theory. 


\section{Nonequilibrium field theory}

As we have already seen several times, the equlibrium field theory gives us also information on dynamical properties of the gas by means of the substitution $\omega_{n} \rightarrow-i \omega$ or equivalently $\tau \rightarrow i t$. However, the Popov theory of Bose-Einstein condensation has shown us that we have to be very careful with this procedure, because we may not always end up with the correct physics needed for a discription of a particular experiment. It is for these cases that a truly nonequilibrium ficld theory is required and the development of such a theory is the topic of Section 3.3. Before embarking on that, however, we first consider two important dynamical problems where the equilibrium theory does give us all the answers.

\subsection{Macroscopic quantum tunneling of a condensate}

In a Bose gas with effectively attractive interactions, i.e., with a negative scattering length $a$, a condensate will always have the tendency to collapse to a high density state due to the gain in encrgy that can be obtained in this way [44]. The most important question in this context is, therefore. if a condensate can exist sufficiently long to be experimentally observed. Neglecting the variation of the noncondensate density on the size of the condensate $[45,46]$, we know from the Popov theory that the dynamics of the collapse is, apart from an unimportant shift in the chemical potential. determined by the Gross-Pitaevskii equation [25]

$$
\begin{aligned}
i \hbar \frac{\partial}{\partial t}\langle\phi(\mathbf{x}, t)\rangle & = \\
& \left\{-\frac{\hbar^{2} \nabla^{2}}{2 m}+V^{\mathrm{ex}}(\mathbf{x})-\mu+\frac{4 \pi a \hbar^{2}}{m}|\langle\phi(\mathrm{x}, t)\rangle|^{2}\right\}\langle\phi(\mathrm{x}, t)\rangle .
\end{aligned}
$$

More precisely, this determines only the semiclassical dynamics. If we also want to study the quantum fluctuations, which is necessary if we are also interested in how the condensate tunnels through the macroscopic energy barrier, it is most convenient to calculate the grand canonical partition function of the condensate [47]. Quantizing the Gross-Pitaevskii equation we obtain for this partition function the functional integral

$$
Z(\mu)=\int \mathrm{d}\left[\phi^{*}\right] \mathrm{d}[\phi] \exp \left\{-\frac{1}{\hbar} S\left[\phi^{*}, \phi\right]\right\} .
$$

over the complex field $\phi(\mathbf{x}, \tau)$ and with the Euclidian action

$$
\begin{aligned}
S\left[\phi^{*}, \phi\right]= & \int_{0}^{\hbar / \beta} \mathrm{d} \tau \int \mathrm{d} \mathbf{x} \phi^{*}(\mathbf{x}, \tau)\left(\hbar \frac{\partial}{\partial \tau}+\frac{\hbar^{2} \nabla^{2}}{2 m}\right. \\
& \left.+V^{\mathrm{ex}}(\mathbf{x})-\mu+\frac{2 \pi a \hbar^{2}}{m}|\phi(\mathbf{x}, \tau)|^{2}\right) \phi(\mathbf{x}, \tau) .
\end{aligned}
$$


As always for Bose systems, the integration is only over fields that are periodic on the imaginary time axis.

Although it has recently been shown by Freire and Arovas that the tunneling process can also be studied in terms of the complex field $\phi(\mathbf{x}, \tau)$ [48]. we believe that it leads to somewhat more physical insight if we use instead the fields $\rho(\mathbf{x}, \tau)$ and $\theta(\mathbf{x}, \tau)$ that correspond to the density and phase fluctuations of the condensate, respectively. They are introduced by performing the canonical variable transformation [33]

$$
\phi(\mathbf{x}, \tau)=\sqrt{\rho(\mathbf{x}, \tau)} \mathrm{e}^{i \theta(\mathbf{x}, \tau)}
$$

in the functional integral for the partition function. As a result we find

$$
Z(\mu)=\int \mathrm{d}[\rho] \mathrm{d}[\theta] \exp \left\{-\frac{1}{\hbar} S[\rho, \theta ; \mu]\right\},
$$

with

$$
\begin{aligned}
S[\rho, \theta ; \mu]= & \int_{0}^{\hbar \beta} \mathrm{d} \tau \int \mathrm{d} \mathbf{x}\left(i \hbar \rho(\mathbf{x}, \tau) \frac{\partial \theta(\mathbf{x}, \tau)}{\partial \tau}\right. \\
& +\frac{\hbar^{2} \rho(\mathbf{x}, \tau)}{2 m}(\nabla \theta(\mathbf{x}, \tau))^{2}+\frac{\hbar^{2}}{8 m \rho(\mathbf{x}, \tau)}(\nabla \rho(\mathbf{x}, \tau))^{2} \\
& \left.+V^{\operatorname{ex}}(\mathbf{x}) \rho(\mathbf{x}, \tau)-\mu \rho(\mathbf{x}, \tau)+\frac{2 \pi a \hbar^{2}}{m} \rho^{2}(\mathbf{x}, \tau)\right) .
\end{aligned}
$$

Next, we notice that this action is only quadratic in the phase fluctuations. The field $\theta(\mathrm{x}, \tau)$ can therefore be integrated over exactly, because it only involves the evaluation of a Gaussian integral.

Compared to ordinary Gaussian integrals there is, however, one slight complication which is associated with the fact that $\theta(\mathbf{x}, \tau)$ are phase variables. This implies that the periodicity of the original field $\phi(\mathbf{x}, \tau)$ only constraints the phase field $\theta(\mathrm{x}, \tau)$ to be periodic up to a multiple of $2 \pi$. To evaluate the grand canonical partition function in equation (3.4) we must therefore first integrate over all fields $\theta(\mathbf{x}, \tau)$ that obey the boundary condition $\theta(\mathbf{x}, h \beta)=\theta(\mathbf{x}, 0)+2 \pi j$ and subsequently sum over all possible integers $j$. Because these different boundary conditions only affect the zero-momentum part of $\theta(\mathrm{x}, \tau)$ we first have to evaluate the sum

$$
\sum_{j} \int^{\theta_{\mathbf{0}}(\hbar \beta)=\theta_{0}(0)+2 \pi j} \mathrm{~d}\left[\theta_{\mathbf{0}}\right] \exp \left\{-i \int_{0}^{\hbar \beta} \mathrm{d} \tau N_{\mathbf{0}}(\tau) \frac{\partial \theta_{\mathbf{0}}(\tau)}{\partial \tau}\right\},
$$

with $N_{0}(\tau)=\int \mathrm{d} \mathbf{x} \rho(\mathrm{x}, \tau)$ the number of condensate particles. After performing a partial integration on the integral in the exponent, we can carry 
out the path integration over $\theta_{0}(\tau)$ to obtain

$$
\sum_{j} \mathrm{e}^{2 \pi i N_{\mathbf{0}} j} \delta\left[\frac{\partial N_{\mathbf{0}}(\tau)}{\partial \tau}\right]
$$

As expected, the integration over the global phase of the condensate leads to the constraint of a constant number of condensate particles, i.e... $N_{\mathbf{0}}(\tau)=$ $N_{0}$. Moreover, we have $\sum_{j} \mathrm{e}^{2 \pi i N_{0} j}=\sum_{j} \delta\left(N_{0}-j\right)$, which restricts the number of condensate particles to an integer. Putting all these results together. we see that the integration over the zero-momentum part of $\rho(\mathbf{x}, \tau)$ is only a sum over the number of condensate particles and we have that

$$
Z(\mu)=\sum_{N_{0}} \mathrm{e}^{\beta \mu N_{0}} Z_{N_{0}}
$$

Here we introduced the canonical partition function of the condensate, which is apparently equal to the functional integral

$$
Z_{N_{0}}=\int \mathrm{d}[\rho] \mathrm{d}[\theta] \exp \left\{-\frac{1}{\hbar} S[\rho, \theta ; 0]\right\}
$$

over all the nonzero momentum components of the density and phase fields.

The integration over the nonzero momentum components of the phase field $\theta(\mathbf{x}, \tau)$ is easily performed, because it now involves an ordinary Gaussian integral. Introducing the Green's function for the phase fluctuations $G\left(\mathrm{x}, \mathrm{x}^{\prime} ; \rho\right)$ by

$$
\frac{\hbar}{m}\left((\nabla \rho) \cdot \nabla+\rho \nabla^{2}\right) G\left(\mathrm{x}, \mathrm{x}^{\prime} ; \rho\right)=\delta\left(\mathrm{x}-\mathrm{x}^{\prime}\right),
$$

we immediately obtain the desired effective action for the density field

$$
\begin{aligned}
S^{\mathrm{eff}}[\rho]= & \int_{0}^{\hbar \beta} \mathrm{d} \tau \int \mathrm{d} \mathbf{x} \int \mathrm{d} \mathbf{x}^{\prime}\left(-\frac{\hbar}{2} \frac{\partial \rho(\mathbf{x}, \tau)}{\partial \tau} G\left(\mathbf{x}, \mathbf{x}^{\prime} ; \rho\right) \frac{\partial \rho\left(\mathbf{x}^{\prime}, \tau\right)}{\partial \tau}\right) \\
& +\int_{0}^{\hbar \beta} \mathrm{d} \tau \int \mathrm{d} \mathbf{x}\left(\frac{\hbar^{2}}{8 m \rho(\mathbf{x}, \tau)}(\nabla \rho(\mathbf{x}, \tau))^{2}\right. \\
& \left.+V^{\operatorname{ex}}(\mathbf{x}) \rho(\mathbf{x}, \tau)+\frac{2 \pi a \hbar^{2}}{m} \rho^{2}(\mathbf{x}, \tau)\right) .
\end{aligned}
$$

Being an action for the density fluctuations of the condensate, $S^{\text {eff }}[\rho]$ also describes all the collisionless modes of the condensate. This is important for our purposes, because the mode which becomes unstable first, determines precisely how the condensate collapses. Moreover, it determines the probability with which the collapse is going to take place, both for quantum and thermal fluctuations, since the energy barrier is smallest in that direction of 
the configuration space. It should be noted that as long as we can neglect the interaction between the condensate and the thermal cloud, the action $S^{\mathrm{eff}}[\rho]$ describes also the collective modes of a gas with positive scattering length. For various other theoretical approaches that have been applied under these conditions see, for example, references $[29,30,49-55]$. The actual measurcments of the collective mode frequencies have been performed by Jin et al. [26] and Mewes et al. [27] and are at sufficiently low temperatures indeed in good agreement with the theoretical predictions $[35,36]$. We expect the same to be true for a gas with cffectively attractive interactions and, thereforc, the action $S^{\text {eff }}[\rho]$ to be a good starting point for the following discussion.

To obtain the collisionless modes explicitly we consider first the case of an ideal Bose gas by putting $a=0$. For the ideal Bose gas we expect the gaussian profile

$$
\rho(\mathbf{x} ; q(\tau))=N_{\mathbf{0}}\left(\frac{1}{\pi q^{2}(\tau)}\right)^{3 / 2} \exp \left(-\frac{\mathbf{x}^{2}}{q^{2}(\tau)}\right)
$$

to describe an exact mode of the condensate. The reason is that in the noninteracting case we can make a density fluctuation by taking one particle from the condensate and putting that in one of the excited states of the external potential. The corresponding density fluctuation obeys

$$
\delta \rho(\mathbf{x}, t) \propto \mathrm{e}^{-i\left(\epsilon_{\mathbf{n}}-\epsilon_{\mathbf{0}}\right) t / \hbar} \chi_{\mathbf{n}}^{*}(\mathbf{x}) \chi_{\mathbf{0}}(\mathbf{x}) .
$$

For the experimentally relevant case of an isotropic harmonic oscillator [11] it is more convenient to use instead of the cartesian quantum numbers $\mathbf{n}$, the two angular momentum quantum numbers $\ell$ and $m$ and the quantum number $n$ that counts the number of nodes in the radial wave function $\chi_{n \ell}(x)$. The density fluctuation then becomes

$$
\delta \rho(\mathbf{x}, t) \propto \mathrm{e}^{-i(2 n+\ell) \omega t} \chi_{n \ell}(x) Y_{\ell m}^{*}(\hat{\mathbf{x}}) \frac{\mathrm{e}^{-x^{2} / 2 l^{2}}}{\left(\pi l^{2}\right)^{3 / 4}},
$$

with $\epsilon_{n \ell m}-\epsilon_{000}=(2 n+\ell) \hbar \omega$ the excitation energy and $l=(\hbar / m \omega)^{1 / 2}$ the size of the condensate wave function. Comparing this now with the expansion of the gaussian profile in equation (3.10) around the groundstate density profile, which is obtained by substituting $q(\tau)=l+\delta q(\tau)$, we find that

$$
\delta \rho(\mathbf{x}, \tau)=-\sqrt{6} N_{0} \frac{\delta q(\tau)}{l} \chi_{10}(x) Y_{00}^{*}(\hat{\mathbf{x}}) \frac{\mathrm{e}^{-x^{2} / 2 l^{2}}}{\left(\pi l^{2}\right)^{3 / 4}}
$$

has precisely the same form as a density fluctuation in which one particle is taken from the condensate and put into the harmonic oscillator state 
with quantum numbers $(n \ell m)=(100)$. The frequency of this so-called "breathing" mode described by the gaussian density profile must therefore be equal to $2 \omega$.

To proof that this is indeed correct, we need to evaluate the effective action $S^{\mathrm{eff}}[\rho]$, and hence the Green's function $G\left(\mathbf{x}, \mathbf{x}^{\prime} ; \rho\right)$, for a gaussian density profile. Substituting such a profile in equation (3.8) immediately leads to $G\left(\mathbf{x}, \mathbf{x}^{\prime} ; \rho\right)=G\left(\mathbf{x}, \mathbf{x}^{\prime} ; q\right) / \rho\left(\mathbf{x}^{\prime} ; q\right)$, with

$$
\frac{\hbar}{m}\left(-\frac{2}{q^{2}} \mathbf{x} \cdot \nabla+\nabla^{2}\right) G\left(\mathrm{x}, \mathrm{x}^{\prime} ; q\right)=\delta\left(\mathrm{x}-\mathrm{x}^{\prime}\right) .
$$

The latter equation can be solved, if we can solve the eigenvalue problem

$$
\left(\nabla^{2}-\frac{2 x}{q^{2}} \frac{\partial}{\partial x}\right) \xi(\mathbf{x})=\lambda \xi(\mathbf{x}) .
$$

This turns out to be an easy task, because substituting

$$
\xi_{n \ell m}(\mathbf{x})=\xi_{n \ell}(x) \frac{\mathrm{e}^{x^{2} / 2 q^{2}}}{x} Y_{\ell m}(\hat{\mathbf{x}})
$$

gives essentially the radial Schrödinger equation for an isotropic harmonic oscillator with frequency $\omega_{q}=\hbar / m q^{2}$, i.e.,

$$
-\frac{2 m}{\hbar^{2}}\left(-\frac{\hbar^{2}}{2 m} \frac{\partial^{2}}{\partial x^{2}}+\frac{1}{2} m \omega_{q}^{2} x^{2}+\frac{\hbar^{2} \ell(\ell+1)}{2 m x^{2}}-\frac{3}{2} \hbar \omega_{q}\right) \xi_{n \ell}(x)=\lambda_{n \ell} \xi_{n \ell}(x) .
$$

The desired eigenfunctions are therefore $\xi_{n \ell m}(\mathbf{x} ; q)=\varphi_{n \ell m}(\mathbf{x}) \mathrm{c}^{x^{2}} / 2 q^{2}$, with $\varphi_{n \ell m}(\mathbf{x})$ the properly normalized harmonic oscillator states with the energies $(2 n+\ell+3 / 2) \hbar \omega_{q}$, and the corresponding eigenvalues are $\lambda_{n \ell}(q)=$ $-2(2 n+\ell) / q^{2}$. Introducing finally the "dual" eigenfunctions $\bar{\xi}_{n \ell m}(\mathbf{x} ; q) \equiv$ $\varphi_{n \ell m}^{*}(\mathbf{x}) \mathrm{e}^{-x^{2} / 2 q^{2}}$, the Green's function $G\left(\mathbf{x}, \mathbf{x}^{\prime} ; q\right)$ is given by

$$
G\left(\mathbf{x}, \mathbf{x}^{\prime} ; q\right)=\sum_{n \ell m}^{\prime} \xi_{n \ell m}(\mathbf{x} ; q) \frac{m}{\hbar \lambda_{n \ell}(q)} \bar{\xi}_{n \ell m}\left(\mathbf{x}^{\prime} ; q\right) .
$$

Note that the prime on the summation sign indicates that the sum is over all quantum numbers except $(\mathrm{n} \ell \mathrm{m})=(000)$. The latter is excluded because the associated eigenfunction $\xi_{000}(\mathbf{x} ; q)$ is just a constant and thus does not contribute to $G\left(\mathrm{x}, \mathrm{x}^{\prime} ; \rho\right)$, which is defined as the Green's function for all phase fluctuations with nonvanishing momenta. 
Putting all these results together, we see that the dynamics of the collective variable $q(\tau)$ is determined by the action

$$
\begin{aligned}
S^{\mathrm{eff}}[q] & =\int_{0}^{h \beta} \mathrm{d} \tau\left\{\frac{3 m N_{0}}{4}\left(\frac{\mathrm{d} q}{\mathrm{~d} \tau}\right)^{2}+N_{0}\left(\frac{3 \hbar^{2}}{4 m q^{2}}+\frac{3}{4} m \omega^{2} q^{2}\right)\right\} \\
& \equiv \int_{0}^{h \beta} \mathrm{d} \tau\left\{\frac{1}{2} m^{*}\left(\frac{\mathrm{d} q}{\mathrm{~d} \tau}\right)^{2}+V(q)\right\}
\end{aligned}
$$

that is equivalent to the action of a particle with effective mass $m^{*}=$ $3 m N_{\mathbf{0}} / 2$ in a potential $V(q)=N_{\mathbf{0}}\left(3 \hbar^{2} / 4 m q^{2}+3 m \omega^{2} q^{2} / 4\right)$. As expected from our previous remarks, this potential has a minimum for $q=l$ and can be expanded near its minimum as

$$
V(q) \simeq \frac{3}{2} N_{\mathbf{0}} \hbar \omega+\frac{1}{2} m^{*}(2 \omega)^{2}(\delta q)^{2} .
$$

It thus comfirms that the gaussian profile describes a breathing mode with frequency $2 \omega$ around an equilibrium density profile that is given by $\rho(\mathbf{x} ; l)=$ $N_{0}\left|\chi_{000}(\mathrm{x})\right|^{2}$.

Our next task is to investigate how interactions affect this result. Considering again only Gaussian density profiles, the action $S^{\text {eff }}[q]$ is again that of a particle with effective mass $m^{*}=3 m N_{0} / 2$ but now in the potential [56]

$$
V(q)=N_{0}\left(\frac{3 \hbar^{2}}{4 m q^{2}}+\frac{3}{4} m \omega^{2} q^{2}-\frac{N_{0}}{\sqrt{2 \pi}} \frac{\hbar^{2}|a|}{m q^{3}}\right) .
$$

The physically most important feature of this potential is that it is unbounded from below, since $V(q) \rightarrow-\infty$ if $q \downarrow 0$. Hence, the condensate indeed always has the tendency to collapse to the high-density state $\lim _{q\rfloor 0} \rho(\mathbf{x} ; q)=N_{0} \delta(\mathbf{x})$. However, if the number of condensate particles is sufficiently small, or more precisely if [57]

$$
N_{0}<\frac{2 \sqrt{2 \pi}}{5^{5 / 4}} \frac{l}{|a|} \simeq 0.68 \frac{l}{|a|},
$$

the condensate has to overcome a macroscopic energy barrier before it can collapse. Under these conditions the condensate is therefore really metastable and can in principle be observed experimentally. The most important question in this respect is of course: How metastable is the condensate? Within the gaussian approximation this question is easily answered, because then the dynamics of the condensate is equivalent to the dynamics of a particle in an unstable potential, as we have just seen. We therefore only need to evaluate the WKB-expression for the tunnneling rate [58] and compare this to the rate of decay due to thermal fluctuations by calculating 
also the height of the energy barrier. The outcome of this comparison for the conditions of the experiment with atomic ${ }^{7} \mathrm{Li}$ is presented in reference [59] and shows that, for the relatively high temperatures $T \gg h w / k_{B}$ that have been obtained thusfar [11], the decay by means of thermal fluctuations over the energy barrier is the dominant decay mechanism of the condensate.

More important for our purposes, however. is that sufficiently close to the maximum number of condensate particles $N_{\max }$ the collective decay of the condensate discussed above is always much more probable than the decay due to two and three-body collisions that lead to a spin-flip or the formation of ${ }^{7} \mathrm{Li}$ molecules. respectively. As a result the collapse of the condensate should be observable within the finite lifetime of the gas. In fact, on the basis of this separation of time scales we expect the condensate to go through a number of growth and collapse cycles [59,60]. Physically this picture arises as follows. Starting from a gas with a number of atoms $N \gg N_{\text {max }}$, the condensate will initially grow as a response to evaporative cooling. However, if the number of condensate atoms starts to come close to $N_{\max }$, the condensate fluctuates over the energy barrier and collapses in a very short time of $\mathcal{O}(1 / \omega)$ [61]. During the collapse the condensate density increases rapidly and two and three-body inclastic processes quickly remove almost all the atoms from the condensate. After this has occurred the condensate grows again from the noncondensed part of the gas and a new growth and collapse cycle begins. It is only after many of these cycles that enough atoms are removed for the gas to relax to an equilibrium with a number of condensate particles that is less than $N_{\max }$. This is shows quantitatively in Figure 16 for the experimental conditions of interest.

A final issue which needs to be addressed at this point is the actual dynamics of the collapse and, in particular, how we must include the effect of the inclastic growth and decay processes on this dynamics. Unfortunately, the inclusion of these effects is rather complicated and we need the results of Section 3.4 to be able to correctly address this problem. It is, however, of some interest because Sackett et al. have recently observed that after a single collapse there remains a remnant of the condensate with about $10 \%$ of the initial number of atoms [62]. At present, it is an important open problem to theoretically understand the magnitude of this remnant.

\subsection{Phase diffusion}

As we have just seen explicitly, a particularly interesting consequence of the finite size of the gas is that quantum fluctuations play a much more important role. Although this is especially true for the case of attractive interactions that we considered in Section 3.1, it is also true for a Bose gas with repulsive interactions. A striking example in this respect is the phenomenon of phase "diffusion", which was recently discussed by Lewenstein and You [32]. We rederive their results for a trapped Bose gas in a 


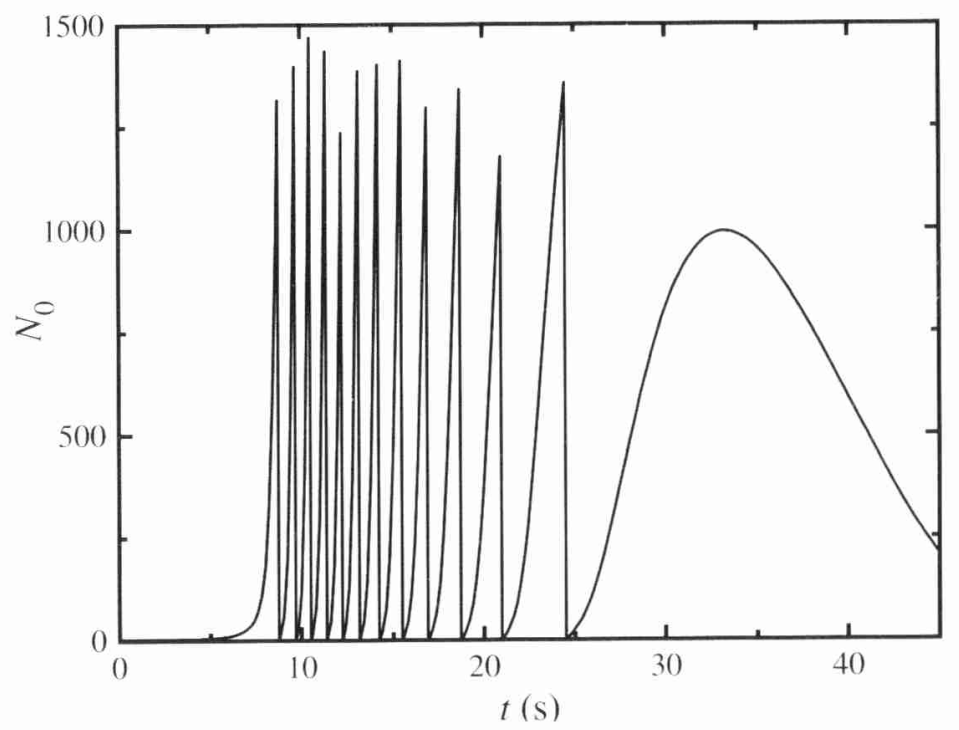

Fig. 16. Typical evolution of condensate number $N_{0}$ in response to evaporative cooling. The long-time decay of the condensate is due to two and three-body inelastic collisions.

moment, but first consider also the same phenomenon for a neutral and homogeneous superconductor. In this manner it is possible to bring out the physics involved more clearly.

Using the approach of Section 2.8.3, it can be shown that at zero temperature the dynamics of the superconducting order parameter, i.e., the BCS gap parameter $\Delta(\mathbf{x}, t)$ that is proportional to the wave function of the condensate of Cooper pairs, is in a good approximation determined by a time-dependent Ginzburg-Landau theory $[41,63,64]$ with the action

$$
\begin{aligned}
S^{\mathrm{eff}}\left[\Delta^{*}, \Delta\right]= & \frac{N(0)}{4} \int \mathrm{d} t \int \mathrm{d} \mathrm{x}\left\{\frac{\hbar^{2}}{\left|\Delta_{0}\right|^{2}}\left|\frac{\partial \Delta}{\partial t}\right|^{2}\right. \\
& \left.-\frac{\hbar^{2} v_{\mathrm{F}}^{2}}{3\left|\Delta_{0}\right|^{2}}|\nabla \Delta|^{2}+2|\Delta|^{2}\left(1-\frac{|\Delta|^{2}}{2\left|\Delta_{0}\right|^{2}}\right)\right\},
\end{aligned}
$$

where $N(0)$ is the density of states for one spin projection at the Fermi energy $\epsilon_{\mathrm{F}}=m v_{\mathrm{F}}^{2} / 2$ and $\Delta_{0}$ is the equilibrium value of the order parameter [65]. Writing the complex order parameter in terms of an amplitude and a phase, we immediately observe that the amplitude fluctuations are gapped [66] and can, therefore, be safely neglected at large length scales. The long-wavelength dynamics of the superconductor is thus dominated by 
the phase fluctuations, according to the action

$$
S^{\mathrm{eff}}[\theta]=\frac{N(0) \hbar^{2}}{4} \int \mathrm{d} t \int \mathrm{d} \mathbf{x}\left\{\left(\frac{\partial \theta}{\partial t}\right)^{2}-\frac{v_{\mathrm{F}}^{2}}{3}(\nabla \theta)^{2}\right\} .
$$

This also implies that the global phase $\theta_{\mathbf{0}}(t)=\int \mathrm{d} \mathbf{x} \theta(\mathrm{x}, t) / V$ of the superconductor has a dynamics that is governed by

$$
S^{\text {eff }}\left[\theta_{\mathbf{0}}\right]=\frac{N(0) N \hbar^{2}}{4 n} \int \mathrm{d} t\left(\frac{\mathrm{d} \theta_{0}}{\mathrm{~d} t}\right)^{2} .
$$

using the fact that the total volume $V$ of the system is given by $N / n$.

Up to now our discussion has again been semiclassical. To consider also the quantum fluctuations, we have to quantize this theory by applying the usual rules of quantum mechanics. Doing so, we find that the wave function of the overall phase obeys a Schrödinger equation

$$
i \hbar \frac{\partial}{\partial t} \Psi\left(\theta_{0} ; t\right)=-\frac{n}{N(0) N} \frac{\partial^{2}}{\partial \theta_{0}^{2}} \Psi\left(\theta_{0} ; t\right) .
$$

with a "diffusion" constant that can easily be shown to be equal to the quantity $(2 / \hbar) \partial \epsilon_{\mathrm{F}} / \partial N[67]$ and is, most importantly for our purposes, proportional to $1 / N$. In the thermodynamic $\operatorname{limit} N \rightarrow \infty$ a state with a well defined stationary phase is clearly a solution and we are then dealing with a system having a spontaneously broken $U(1)$ symmetry. However, for a finite (and fixed) number of particles the global phase camnot be well defined at all times and always has to "diffuse" in accordance with the above Schrödinger equation. Note also that in the groundstate the phase is fully undetermined and $\left|\Psi\left(\theta_{0} ; t\right)\right|^{2}=1 / 2 \pi$. Maybe surprisingly, the same calculation is somewhat more complicated for a Bose gas because the amplitude fluctuations of the order parameter cannot be neglected even at the largest length scales. However, taking these amplitude fluctuations into account properly, we nevertheless arrive at an action that is equivalent to equation (3.23) and hence again leads to the phenomenon of phase "diffusion".

We start again from the action $S[\rho, \theta ; \mu]$ for the condensate. The difference with the previous subsection is, however, that now we are not so much interested in the dynamics of the density but in the phase dynamics instead. Therefore we now want to integrate over the density field $\rho(\mathbf{x}, \tau)$. This cannot be done exactly and we therefore here consider only the strong-coupling limit, which was also treated by Lewenstein and You [32]. In that limit we are allowed to neglect the gradient of the average density profile [68] and 
the action $S[\rho, \theta ; \mu]$ is for the longest wavelengths well approximated by

$$
\begin{aligned}
S[\rho, \theta ; \mu]= & \int_{0}^{\hbar \beta} \mathrm{d} \tau \int \mathrm{d} \mathbf{x}\left(i \hbar \rho(\mathbf{x}, \tau) \frac{\partial \theta(\mathbf{x}, \tau)}{\partial \tau}\right. \\
& \left.+V^{\operatorname{ex}}(\mathbf{x}) \rho(\mathbf{x}, \tau)-\mu \rho(\mathbf{x}, \tau)+\frac{2 \pi a \hbar^{2}}{m} \rho^{2}(\mathbf{x}, \tau)\right) .
\end{aligned}
$$

In equilibrium the average density profile of the condensate thus obeys

$$
\langle\rho(\mathrm{x})\rangle=\frac{m}{4 \pi a \hbar^{2}}\left(\mu-V^{\mathrm{ex}}(\mathrm{x})\right) \Theta\left(\mu-V^{\mathrm{ex}}(\mathrm{x})\right) .
$$

Performing now the shift $\rho(\mathbf{x}, \tau)=\langle\rho(\mathbf{x})\rangle+\delta \rho(\mathbf{x}, \tau)$, we find for the zeromomentum part of the action [69]

$$
S\left[\delta N_{0}, \theta_{\mathbf{0}} ; \mu\right]=\hbar \beta E_{\mathbf{0}}(\mu)+\int_{0}^{h \beta} \mathrm{d} \tau\left(i \hbar \delta N_{\mathbf{0}} \frac{\mathrm{d} \theta_{\mathbf{0}}}{\mathrm{d} \tau}+\frac{2 \pi a \hbar^{2}}{m V_{\mathbf{0}}(\mu)}\left(\delta N_{\mathbf{0}}\right)^{2}\right),
$$

where $E_{0}(\mu)$ and $V_{0}(\mu)$ correspond, respectively, to the energy and the volume of the condensate in the so-called Thomas-Fermi approximation [70]. Moreover, $\delta N_{0}(\tau)=\int \mathrm{d} \mathbf{x} \delta \rho(\mathbf{x}, \tau)$ represents the fluctuations in the total number of condensate particles in that same approximation, implying that the density fluctuations $\delta \rho(\mathrm{x}, \tau)$ are only nonzero in that region of space where the condensate density does not vanish.

Performing now the integration over the number fluctuations $\delta N_{\mathbf{0}}(\tau)$ and the usual Wick rotation to real times $\tau \rightarrow$ it, we immediately see that the effective action for the global phase of the condensate has precisely the same form as in equation (3.23), i.e.,

$$
S^{\mathrm{eff}}\left[\theta_{0}: \mu\right]=\frac{m V_{0}(\mu)}{8 \pi a} \int \mathrm{d} t\left(\frac{\mathrm{d} \theta_{0}}{\mathrm{~d} t}\right)^{2} .
$$

The appropriate "diffusion" constant is therefore equal to $2 \pi a h / m V_{\mathbf{0}}(\mu)$, which can easily be shown to be equal to $(1 / 2 \hbar) \partial \mu / \partial N_{0}$ if we make use of the fact that in the Thomas-Fermi approximation the chemical potential obeys $\mu=m \omega^{2} R_{\mathrm{TF}}^{2} / 2$ and the radius of the condensate is given by $R_{\mathrm{TF}}=$ $\left(15 a \hbar^{2} N_{0} / m^{2} \omega^{2}\right)^{1 / 5}[56]$. Hence, the "diffusion" constant is proportional to $1 / N_{0}^{3 / 5}$. Note that if the condensate where contained in a box the "diffusion" constant would be proportional to $1 / N_{0}$ instead. It is important to note also that, in contrast to the case of a fermionic superfluid, we have to integrate over the amplitude fluctuations of the order parameter to arrive at a quadratic action for the phase fluctuations. This leads to the important conclusion that for a bosonic superfluid it is impossible to be in a state with only phase fluctuations and no density fluctuations, even at the largest length scales. 


\subsection{Quantum kinetic theory}

For simplicity we restrict ourselves for the rest of this course to the case of a doubly-polarized atomic Bose gas, which for most practical purposes can be seen as a gas of spin-less bosons [71]. Once we have understood the quantum kinetic theory for this particular case, however. we can easily generalize to multi-component Bose gases that are receiving considerable attention at present [72]. Furthermore, the same methods can also be used for fermionic gases and have recently already been applied to the problem of Cooper-pair formation in a two-component fermion gas [73].

\subsubsection{Ideal Bose gas}

In textbooks an ideal Bose gas is generally discussed in terms of the average occupation numbers of the one-particle states $\chi_{\mathbf{n}}(\mathbf{x})$ [74]. Given the density matrix $\hat{\rho}\left(t_{0}\right)$ of the gas at an initial time $t_{0}$, these occupation numbers obey

$$
N_{\mathbf{n}}(t)=\operatorname{Tr}\left[\hat{\rho}\left(t_{0}\right) \hat{\psi}_{\mathbf{n}}^{\dagger}(t) \hat{\psi}_{\mathbf{n}}(t)\right],
$$

with $\hat{\psi}_{\mathbf{n}}^{\dagger}(t)$ and $\hat{\psi}_{\mathbf{n}}(t)$ the usual (Heisenberg picture) creation and annihilation operators of second quantization, respectively. Because the Hamiltonian of the gas

$$
\hat{H}=\sum_{\mathbf{n}} \epsilon_{\mathbf{n}} \hat{\psi}_{\mathbf{n}}^{\dagger}(t) \hat{\psi}_{\mathbf{n}}(t)
$$

commutes with the number operators $\hat{N}_{\mathbf{n}}(t)=\hat{\psi}_{\mathbf{n}}^{\dagger}(t) \hat{\psi}_{\mathbf{n}}(t)$, the nonequilibrium dynamics of the system is trivial and the average occupation numbers are at all times equal to their value at the initial time $t_{0}$. If we are also interested in fluctuations, it is convenient to introduce the eigenstates of the number operators, i.e.,

$$
\left|\left\{N_{\mathbf{n}}\right\} ; t\right\rangle=\prod_{\mathbf{n}} \frac{\left(\hat{\psi}_{\mathbf{n}}^{\dagger}(t)\right)^{N_{\mathbf{n}}}}{\sqrt{N_{\mathbf{n}} !}}|0\rangle,
$$

and to consider the full probability distribution

$$
P\left(\left\{N_{\mathbf{n}}\right\} ; t\right)=\operatorname{Tr}\left[\hat{\rho}\left(t_{0}\right)\left|\left\{N_{\mathbf{n}}\right\} ; t\right\rangle\left\langle\left\{N_{\mathbf{n}}\right\} ; t\right|\right],
$$

which is again independent of time for an ideal Bose gas. The average occupation numbers are then determined by

$$
N_{\mathbf{n}}(t)=\sum_{\left\{N_{\mathbf{n}}\right\}} N_{\mathbf{n}} P\left(\left\{N_{\mathbf{n}}\right\} ; t\right)
$$


and the fluctuations can be obtained from similar expressions.

As indicated in Section 3.2, we are also interested in the phase dynamies of the gas. In analogy with the occupation number representation in equation (3.32), we can now obtain a completely different description of the Bose gas by making use of coherent states and considering the probability distribution $P\left[\phi^{*}, \phi ; t\right]$. Although we expect that this probability distribution is again independent of time, let us nevertheless proceed to derive its equation of motion in a way that can be generalized lateron when we consider an interacting Bose gas. First, we use that

$$
P\left[\phi^{*}, \phi ; t\right]=\int \mathrm{d}\left[\phi_{0}^{*}\right] \mathrm{d}\left[\phi_{0}\right] P\left[\phi_{0}^{*}, \phi_{0} ; t_{0}\right] \frac{\left|\left\langle\phi ; t \mid \phi_{0} ; t_{0}\right\rangle\right|^{2}}{\langle\phi ; t \mid \phi ; t\rangle\left\langle\phi_{0} ; t_{0} \mid \phi_{0} ; t_{0}\right\rangle} .
$$

This is a particularly useful result, because the time dependence is now completely determined by the matrix element $\left\langle\phi ; t \mid \phi_{0} ; t_{0}\right\rangle$ for which the functional integral representation is well known from our discussion in Section 2.3. It is given by a "path" integral over all complex field evolutions $\psi\left(\mathbf{x}, t_{+}\right)=\sum_{\mathbf{n}} \psi_{\mathbf{n}}\left(t_{+}\right) \chi_{\mathbf{n}}(\mathrm{x})$ from $t_{0}$ to $t$. More precisely we have

$$
\left\langle\phi ; t \mid \phi_{0} ; t_{0}\right\rangle=\int_{\psi\left(\mathbf{x}, t_{0}\right)=\phi_{0}(\mathbf{x})}^{\psi^{*}(\mathbf{x}, t)=\phi^{*}(\mathbf{x})} \mathrm{d}\left[\psi^{*}\right] \mathrm{d}[\psi] \exp \left\{\frac{i}{\hbar} S_{+}\left[\psi^{*}, \psi\right]\right\},
$$

with the forward action $S_{+}\left[\psi^{*}, \psi\right]$ given by

$$
\begin{aligned}
S_{+}\left[\psi^{*}, \psi\right]= & \sum_{\mathbf{n}}\left\{-i \hbar \psi_{\mathbf{n}}^{*}(t) \psi_{\mathbf{n}}(t)\right. \\
& \left.+\int_{t_{0}}^{t} \mathrm{~d} t_{+} \psi_{\mathbf{n}}^{*}\left(t_{+}\right)\left(i \hbar \frac{\partial}{\partial t_{+}}-\epsilon_{\mathbf{n}}\right) \psi_{\mathbf{n}}\left(t_{+}\right)\right\} .
\end{aligned}
$$

In the same manner the matrix element $\left\langle\phi ; t \mid \phi_{0} ; t_{0}\right\rangle^{*}=\left\langle\phi_{0} ; t_{0} \mid \phi ; t\right\rangle$ can be written as a "path" integral over all possible field configurations $\psi\left(\mathbf{x}, t_{-}\right)=$ $\sum_{\mathbf{n}} \psi_{\mathbf{n}}\left(t_{-}\right) \chi_{\mathbf{n}}(\mathrm{x})$ evolving backward in time from $t$ to $t_{0}$, i.e.,

$$
\left\langle\phi ; t \mid \phi_{0} ; t_{0}\right\rangle^{*}=\int_{\psi(\mathbf{x}, t)=\phi(\mathbf{x})}^{\psi^{*}\left(\mathbf{x}, t_{0}\right)=\phi_{0}^{*}(\mathbf{x})} \mathrm{d}\left[\psi^{*}\right] \mathrm{d}[\psi] \exp \left\{\frac{i}{\hbar} S_{-}\left[\psi^{*}, \psi\right]\right\},
$$

with a backward action

$$
\begin{aligned}
S_{-}\left[\psi^{*}, \psi\right]= & \sum_{\mathbf{n}}\left\{-i \hbar \psi_{\mathbf{n}}^{*}\left(t_{0}\right) \psi_{\mathbf{n}}\left(t_{0}\right)\right. \\
& \left.+\int_{t}^{t_{0}} \mathrm{~d} t_{-} \psi_{\mathbf{n}}^{*}\left(t_{-}\right)\left(i \hbar \frac{\partial}{\partial t_{-}}-\epsilon_{\mathbf{n}}\right) \psi_{\mathbf{n}}\left(t_{-}\right)\right\} \\
= & \sum_{\mathbf{n}}\left\{-i \hbar \psi_{\mathbf{n}}^{*}(t) \psi_{\mathbf{n}}(t)\right. \\
& \left.+\int_{t}^{t_{0}} \mathrm{~d} t_{-} \psi_{\mathbf{n}}\left(t_{-}\right)\left(-i \hbar \frac{\partial}{\partial t_{-}}-\epsilon_{\mathbf{n}}\right) \psi_{\mathbf{n}}^{*}\left(t_{-}\right)\right\} .
\end{aligned}
$$


Putting all these results together, we see that the probability distribution $P\left[\phi^{*}, \phi ; t\right]$ can in fact be represented by a functional integral over all fields $\psi(\mathbf{x} . t)$ that evolve backwards from $t$ to $t_{0}$ and then forward in time from $t_{0}$ to $t$. Absorbing the factor $P\left[\phi_{0}^{*}, \phi_{0} ; t_{0}\right]$ into the measure of the functional integral, we thus arrive at the desired result that

$$
P\left[\phi^{*}, \phi: t\right]=\int_{\psi(\mathbf{x}, t)=\phi(\mathbf{x})}^{\psi^{*}(\mathbf{x}, t)=\phi^{*}(\mathbf{x})} \mathrm{d}\left[\psi^{*}\right] \mathrm{d}[\psi] \exp \left\{\frac{i}{h} S\left[\psi^{*}, \psi\right]\right\} .
$$

where the total (backward-forward) action in first instance obeys

$$
\begin{aligned}
S\left[\psi^{*}, \psi\right]= & S_{-}\left[\psi^{*}, \psi\right]+S_{+}\left[\psi^{*}, \psi\right]=-i \hbar \sum_{\mathbf{n}}\left(\psi_{\mathbf{n}}^{*}(t) \psi_{\mathbf{n}}(t)-\left|\phi_{\mathbf{n}}\right|^{2}\right) \\
& +\sum_{\mathbf{n}} \int_{\mathcal{C}^{t}} \mathrm{~d} t^{\prime}\left\{\frac { 1 } { 2 } \left(\psi_{\mathbf{n}}^{*}\left(t^{\prime}\right) i \hbar \frac{\partial}{\partial t^{\prime}} \psi_{\mathbf{n}}\left(t^{\prime}\right)\right.\right. \\
& \left.\left.-\psi_{\mathbf{n}}\left(t^{\prime}\right) i \hbar \frac{\partial}{\partial t^{\prime}} \psi_{\mathbf{n}}^{*}\left(t^{\prime}\right)\right)-\epsilon_{\mathbf{n}} \psi_{\mathbf{n}}^{*}\left(t^{\prime}\right) \psi_{\mathbf{n}}\left(t^{\prime}\right)\right\},
\end{aligned}
$$

and the integration along the Schwinger-Keldysh contour $\mathcal{C}^{t}$ is defined by $\int_{\mathcal{C}^{t}} \mathrm{~d} t^{\prime}=\int_{t}^{t_{10}} \mathrm{~d} t-+\int_{t_{1}}^{t} \mathrm{~d} t_{+}[75-77]$. Note also that in equation (3.39) we have explicitly specified the boundary conditions on the functional integral. It is interesting to mention that these boundary conditions are essentially dictated by the topological terms in the action $S\left[\psi^{*}, \psi\right]$, which is a general feature of the path-integral formulation of quantum mechanics due to the fact that the quantum theory should have the correct (scmi)classical limit [78]. Making use of the periodicity of the field $\psi(\mathbf{x}, t)$ on the SchwingerKeldysh contour, the variational principle $\delta S\left[\psi^{*}, \psi\right] / \delta \psi_{\mathbf{n}}^{*}\left(t_{ \pm}\right)=0$ indeed leads not only to the Euler-Lagrange equation

$$
i \hbar \frac{\partial}{\partial t_{ \pm}} \psi_{\mathbf{n}}\left(t_{ \pm}\right)=\epsilon_{\mathbf{n}} \psi_{\mathbf{n}}\left(t_{ \pm}\right)
$$

which agrees with the Heisenberg equation of motion $i \hbar \partial \hat{\psi}_{\mathbf{n}}\left(t_{ \pm}\right) / \partial t_{ \pm}=$ $\left[\hat{\psi}_{\mathbf{n}}\left(t_{ \pm}\right), H\right]$ for the annihilation operators, but also with the appropriate boundary condition $\delta \psi_{\mathbf{n}}^{*}(t)=0$. In the same way we find the complex conjugate results if we require that $\delta S\left[\psi^{*}, \psi\right] / \delta \psi_{\mathbf{n}}\left(t_{ \pm}\right)=0$. Substituting the boundary conditions in equation (3.40) and performing a partial integration, we then finally obtain for the action

$$
S\left[\psi^{*}, \psi\right]=\sum_{\mathbf{n}} \int_{\mathcal{C}^{\dagger}} \mathrm{d} t^{\prime} \psi_{\mathbf{n}}^{*}\left(t^{\prime}\right)\left(i \hbar \frac{\partial}{\partial t^{\prime}}-\epsilon_{\mathbf{n}}\right) \psi_{\mathbf{n}}\left(t^{\prime}\right),
$$

which we for completeness sake also rewrite as

$$
S\left[\psi^{*}, \psi\right]=\int_{\mathcal{C}^{t}} \mathrm{~d} t^{\prime} \int \mathrm{d} \mathbf{x} \psi^{*}\left(\mathbf{x}, t^{\prime}\right)\left(i \hbar \frac{\partial}{\partial t^{\prime}}+\frac{\hbar^{2} \nabla^{2}}{2 m}-V^{\mathbf{e x}}(\mathbf{x})\right) \psi\left(\mathbf{x}, t^{\prime}\right) .
$$


We are now in a position to derive the equation of motion, i.e., the FokkerPlanck equation, for the probability distibution $P\left[\phi^{*}, \phi ; t\right]$. This is most easily achieved by performing the variable transformation $\psi\left(\mathrm{x}, t_{ \pm}\right)=\phi\left(\mathbf{x}, t^{\prime}\right) \pm$ $\xi\left(\mathrm{x}, t^{\prime}\right) / 2$ in equation $(3.39)$. In this manner the fields $\psi\left(\mathbf{x}, t_{-}\right)$and $\psi\left(\mathbf{x}, t_{+}\right)$ that live on the backward and forward branch of the Schwinger-Keldysh contour, respectively, are "projected" onto the real time axis. Moreover, at the same time we effect a separation between the (semi)classical dynamics described by $\phi\left(\mathrm{x}, t^{\prime}\right)$ and the quantum fluctuations determined by $\xi\left(\mathrm{x}, t^{\prime}\right)$. After the transformation we have

$$
P\left[\phi^{*}, \phi ; t\right]=\int_{\phi(\mathbf{x}, t)=\phi(\mathbf{x})}^{\phi^{*}(\mathbf{x}, t)=\phi^{*}(\mathbf{x})} \mathrm{d}\left[\phi^{*}\right] \mathrm{d}[\phi] \int \mathrm{d}\left[\xi^{*}\right] \mathrm{d}[\xi] \exp \left\{\frac{i}{\hbar} S\left[\phi^{*}, \phi ; \xi^{*}, \xi\right]\right\}
$$

with

$$
\begin{aligned}
S\left[\phi^{*}, \phi ; \xi^{*}, \xi\right]= & \sum_{\mathbf{n}} \int_{t_{0}}^{t} \mathrm{~d} t^{\prime}\left\{\phi_{\mathbf{n}}^{*}\left(t^{\prime}\right)\left(i \hbar \frac{\partial}{\partial t^{\prime}}-\epsilon_{\mathbf{n}}\right) \xi_{\mathbf{n}}\left(t^{\prime}\right)\right. \\
& \left.+\xi_{\mathbf{n}}^{*}\left(t^{\prime}\right)\left(i \hbar \frac{\partial}{\partial t^{\prime}}-\epsilon_{\mathbf{n}}\right) \phi_{\mathbf{n}}\left(t^{\prime}\right)\right\} .
\end{aligned}
$$

Becanse this action is linear in $\xi_{\mathbf{n}}\left(t^{\prime}\right)$ and $\xi_{\mathbf{n}}^{*}\left(t^{\prime}\right)$, the integration over the quantum fluctuations leads just to a constraint and we find that

$$
\begin{aligned}
P\left[\phi^{*}, \phi ; t\right]= & \int_{\phi(\mathbf{x}, t)=\phi(\mathbf{x})}^{\phi^{*}(\mathbf{x}, t)=\phi^{*}(\mathbf{x})} \mathrm{d}\left[\phi^{*}\right] \mathrm{d}[\phi] \\
& \times \prod_{\mathbf{n}} \delta\left[\left(-i \frac{\partial}{\partial t^{\prime}}-\frac{\epsilon_{\mathbf{n}}}{\hbar}\right) \phi_{\mathbf{n}}^{*}\left(t^{\prime}\right) \cdot\left(i \frac{\partial}{\partial t^{\prime}}-\frac{\epsilon_{\mathbf{n}}}{\hbar}\right) \phi_{\mathbf{n}}\left(t^{\prime}\right)\right]
\end{aligned}
$$

or equivalently that

$$
P\left[\phi^{*}, \phi ; t\right]=\int \mathrm{d}\left[\phi_{0}^{*}\right] \mathrm{d}\left[\phi_{0}\right] P\left[\phi_{0}^{*}, \phi_{0} ; t_{0}\right] \prod_{\mathbf{n}} \delta\left(\left|\phi_{\mathbf{n}}-\phi_{\mathbf{n}}^{\mathrm{cl}}(t)\right|^{2}\right),
$$

where we introduced the quantity $\phi_{\mathbf{n}}^{c l}(t)$ obeying the semiclassical equation of motion

$$
i \hbar \frac{\partial}{\partial t} \phi_{\mathbf{n}}^{\mathrm{cl}}(t)=\epsilon_{\mathbf{n}} \phi_{\mathbf{n}}^{\mathrm{cl}}(t)
$$

and the initial condition $\phi_{\mathbf{n}}^{\mathrm{cl}}\left(t_{0}\right)=\phi_{0 ; \mathbf{n}}$.

The latter equation is thus solved by $\phi_{\mathbf{n}}^{\mathrm{cl}}(t)=\phi_{0 ; \mathbf{n}} \mathrm{e}^{-i \epsilon_{\mathbf{n}}\left(t-t_{0}\right) / \hbar}$ and we conclude from a simple change of variables in equation (3.47) that for an 
ideal Bose gas $P\left[\phi^{*}, \phi: t\right]=P\left[\phi^{*}, \phi: t_{0}\right]$, as expected. We also see from equation (3.47) that the desired equation of motion for $P\left[\phi^{*}, \phi ; t\right]$ reads

$$
\begin{aligned}
i \hbar \frac{\partial}{\partial t} P\left[\phi^{*}, \phi ; t\right]= & -\left(\sum_{\mathbf{n}} \frac{\partial}{\partial \phi_{\mathbf{n}}} \epsilon_{\mathbf{n}} \phi_{\mathbf{n}}\right) P\left[\phi^{*}, \phi ; t\right] \\
& +\left(\sum_{\mathbf{n}} \frac{\partial}{\partial \phi_{\mathbf{n}}^{*}} \epsilon_{\mathbf{n}} \phi_{\mathbf{n}}^{*}\right) P\left[\phi^{*}, \phi ; t\right] .
\end{aligned}
$$

This is indeed the correct Fokker-Planck equation for an ideal Bose gas [79]. To see that we first consider $\left\langle\phi_{\mathbf{n}}\right\rangle(t)=\int \mathrm{d}\left[\phi^{*}\right] \mathrm{d}[\phi] \phi_{\mathbf{n}} P\left[\phi^{*}, \phi ; t\right]$. Multiplying equation (3.49) with $\phi_{\mathbf{n}}$ and integrating over $\phi(\mathbf{x})$, we easily find after a partial integration that

$$
i \hbar \frac{\partial}{\partial t}\left\langle\phi_{\mathbf{n}}\right\rangle(t)=\epsilon_{\mathbf{n}}\left\langle\phi_{\mathbf{n}}\right\rangle(t) .
$$

which precisely corresponds to the equation of motion of the expectation value $\left\langle\hat{\psi}_{\mathbf{n}}(t)\right\rangle=\operatorname{Tr}\left[\hat{\rho}\left(t_{0}\right) \hat{\psi}_{\mathbf{n}}(t)\right]$ in the operator formalism. Similarly, we find that

$$
i \hbar \frac{\partial}{\partial t}\left\langle\phi_{\mathbf{n}}^{*}\right\rangle(t)=-\epsilon_{\mathbf{n}}\left\langle\phi_{\mathbf{n}}^{*}\right\rangle(t),
$$

in agreement with the resul, ior $\left.\hat{\psi}_{\mathbf{n}}^{\dagger}(t)\right\rangle=\operatorname{Tr}\left[\hat{\rho}\left(t_{0}\right) \hat{\psi}_{\mathbf{n}}^{\dagger}(t)\right]$.

Next we consider the average of $\left|\phi_{\mathbf{n}}\right|^{2}$, for which we immediately obtain

$$
i \hbar \frac{\partial}{\partial t}\left\langle\left|\phi_{\mathbf{n}}\right|^{2}\right\rangle(t)=0 .
$$

We expect this result to be related to the fact that in the operator formalism the occupation numbers $N_{\mathbf{n}}(t)$ are independent of time. Although this turns out to be true, to give the precise relation between $\left\langle\left|\phi_{\mathbf{n}}\right|^{2}\right\rangle(t)$ and $N_{\mathbf{n}}(t)$ is complicated by the fact that at equal times the operators $\hat{\psi}_{\mathbf{n}}(t)$ and $\hat{\psi}_{\mathbf{n}}^{\dagger}(t)$ do not commute. However, the path-integral formulation of quantum mechanics is only capable of calculating time-ordered operator products [80]. In our case this implies that $\left\langle\left|\phi_{\mathbf{n}}\right|^{2}\right\rangle(t)$ is the value at $t^{\prime}=t$ of

$$
\begin{aligned}
& \operatorname{Tr}\left[\hat{\rho}\left(t_{0}\right) T_{\mathcal{C}^{t}}\left(\hat{\psi}_{\mathbf{n}}(t) \hat{\psi}_{\mathbf{n}}^{\dagger}\left(t^{\prime}\right)\right)\right]= \\
& \Theta\left(t, t^{\prime}\right) \operatorname{Tr}\left[\hat{\rho}\left(t_{0}\right) \hat{\psi}_{\mathbf{n}}(t) \hat{\psi}_{\mathbf{n}}^{\dagger}\left(t^{\prime}\right)\right]+\Theta\left(t^{\prime}, t\right) \operatorname{Tr}\left[\hat{\rho}\left(t_{0}\right) \hat{\psi}_{\mathbf{n}}^{\dagger}\left(t^{\prime}\right) \hat{\psi}_{\mathbf{n}}(t)\right],
\end{aligned}
$$

with $T_{\mathcal{C}^{t}}$ the time-ordening operator on the Schwinger-Keldysh contour and $\Theta\left(t, t^{\prime}\right)$ the corresponding Heaviside function. Since the Heaviside function is equal to $1 / 2$ at equal times [81], we conclude that

$$
\left\langle\left|\phi_{\mathbf{n}}\right|^{2}\right\rangle(t)=N_{\mathbf{n}}(t)+\frac{1}{2}
$$


and that equation (3.52) is thus fully consistent with the operator formalism. An intuitive understanding of the relation between $\left\langle\left|\phi_{\mathbf{n}}\right|^{2}\right\rangle(t)$ and the occupation numbers can be obtained by noting that, since all our manipulations up to now have been exact, we expect $\left\langle\left|\phi_{\mathbf{n}}\right|^{2}\right\rangle(t)$ to contain both classical and quantum fluctuations. These correspond precisely to the contributions $N_{\mathbf{n}}(t)$ and $1 / 2$, respectively.

Finally we need to discuss the stationary solutions of the Fokker-Planck equation. It is not difficult to show that any functional that only depends on the amplitudes $\left|\phi_{\mathbf{n}}\right|^{2}$ is a solution. As it stands the Fokker-Planck equation, therefore, does not lead to a unique equilibrium distribution. This is not surprising, because for an isolated, ideal Bose gas there is no mechanism for redistributing the particles over the various energy levels and thus for relaxation towards equilibrium. However, the situation changes when we allow the bosons in the trap to tumnel back and forth to a reservoir at a temperature $T$. The corrections to the Fokker-Planck equation that are required to describe the physies in this case are considered next. However, to determine these corrections in the most convenient way, we have to slightly generalize the above theory because with the probability distibution $P\left[\phi^{*}, \phi ; t\right]$ we are only able to study spatial. but not temporal correlations in the Bose gas.

To study also those we follow the by now well-known procedure in quantum field theory and construct a generating functional $Z\left[J, J^{*}\right]$ for all (time-ordered) correlation functions. It is obtained by performing two steps. First, we introduce the probability distribution $P_{J}\left[\phi^{*}, \phi ; t\right]$ for a Bose gas in the presence of the extermal currents $J(\mathrm{x}, t)$ and $J^{*}(\mathrm{x}, t)$ by adding to the Hamiltonian the terms

$$
\begin{aligned}
& -\hbar \int \mathrm{d} \mathbf{x}\left(\hat{\psi}(\mathbf{x}, t) J^{*}(\mathbf{x}, t)+J(\mathbf{x}, t) \hat{\psi}^{\dagger}(\mathbf{x}, t)\right)= \\
& \quad-\hbar \sum_{\mathbf{n}}\left(\hat{\psi}_{\mathbf{n}}(t) J_{\mathbf{n}}^{*}(t)+J_{\mathbf{n}}(t) \hat{\psi}_{\mathbf{n}}^{\dagger}(t)\right) .
\end{aligned}
$$

As a result we have

$$
\begin{aligned}
& P_{, J}\left[\phi^{*}, \phi ; t\right]=\int_{\psi(\mathbf{x}, t)=\phi(\mathbf{x})}^{\psi^{*}(\mathbf{x}, t)=\phi^{*}(\mathbf{x})} \mathrm{d}\left[\psi^{*}\right] \mathrm{d}[\psi] \exp \left\{\frac{i}{\hbar} S\left[\psi^{*}, \psi\right]\right\} \\
& \quad \times \exp \left\{i \int_{\mathcal{C}^{t}} \mathrm{~d} t^{\prime} \int \mathrm{dx}\left(\psi\left(\mathbf{x}, t^{\prime}\right) J^{*}\left(\mathbf{x}, t^{\prime}\right)+J\left(\mathbf{x}, t^{\prime}\right) \psi \psi^{*}\left(\mathbf{x}, t^{\prime}\right)\right)\right\} .
\end{aligned}
$$


Second. we integrate this expression over $\phi(\mathrm{x})$ to obtain the desired generating functional. Hence

$$
\begin{aligned}
Z\left[J . J^{*}\right]= & \int \mathrm{d}\left[\phi^{*}\right] \mathrm{d}[\phi] P_{J}\left[\phi^{*}, \phi ; t\right]=\int \mathrm{d}\left[\psi^{*}\right] \mathrm{d}[\psi] \exp \left\{\frac{i}{h} S\left[\psi^{*}, \psi\right]\right\} \\
& \times \exp \left\{i \int_{\mathcal{C} x} \mathrm{~d} t \int \mathrm{dx}\left(\psi(\mathbf{x}, t) J^{*}(\mathbf{x}, t)+J(\mathbf{x}, t) \psi^{*}(\mathbf{x}, t)\right)\right\} .
\end{aligned}
$$

It is important to realize that $Z\left[J, J^{*}\right]$ is indeed independent of the time $t$ because of the fact that $P_{J}\left[\phi^{*}, \phi ; t\right]$ is a probability distribution and thus properly normalized. We are therefore allowed to deform the contour $\mathcal{C}^{t}$ to any closed contour that rums through $t_{0}$. Since we are in principle interested in all times $t \geq t_{0}$, the most convenient choice is the countour that runs backward from infinity to $t_{0}$ and then forwards from to to infinity. This contour is denoted by $\mathcal{C}^{\infty}$ and also called the Schwinger-Keldysh contour in the following because there is in practice never confusion with the more restricted contour $\mathcal{C}^{t}$ that is required when we consider a probability distribution. With this choice it is also clear that $Z\left[J, J^{*}\right]$ is a generating functional. Indeed, equation (3.56) shows explicitly that all time-ordered correlation functions can be obtained by functional differentiation with respect to the currents $J(\mathbf{x}, t)$ and $J^{*}(\mathbf{x}, t)$. We have, for instance, that

$$
\operatorname{Tr}\left[\hat{\rho}\left(t_{0}\right) \hat{\psi}(\mathbf{x}, t)\right]=\left.\frac{1}{i} \frac{\delta}{\delta J^{*}(\mathbf{x}, t)} Z\left[J, J^{*}\right]\right|_{, J, J^{*}=0}
$$

and similarly that

$$
\begin{aligned}
\operatorname{Tr}\left[\hat{\rho}\left(t_{0}\right)\right. & \left.T_{\mathcal{C} x}\left(\hat{\psi}(\mathbf{x}, t) \hat{\psi}^{\dagger}\left(\mathbf{x}^{\prime}, t^{\prime}\right)\right)\right] \\
& =\left.\frac{1}{i^{2}} \frac{\delta^{2}}{\delta J^{*}(\mathbf{x}, t) \delta J\left(\mathbf{x}^{\prime}, t^{\prime}\right)} Z\left[J, J^{*}\right]\right|_{, J, J^{*}=0} .
\end{aligned}
$$

Note that the times $t$ and $t^{\prime}$ always have to be larger or equal to $t_{0}$ for these identities to be valid.

\subsubsection{Ideal Bose gas in contact with a reservoir}

Inspired by the Caldeira-Leggett model for a particle experiencing friction [82], we take for the reservoir an ideal gas of $N$ bosons in a box with volume $V$. The states in this box are labeled by the momentum $\hbar \mathbf{k}$ and equal to $\chi_{\mathbf{k}}(\mathbf{x})=\mathrm{e}^{i \mathbf{k} \cdot \mathbf{x}} / \sqrt{V}$. They have an energy $\epsilon(\mathbf{k})=\hbar^{2} \mathbf{k}^{2} / 2 m+\Delta V^{\mathrm{cx}}$, where $\Delta V^{\text {ex }}$ accounts for a possible bias between the potential energies of a particle in the center of the trap and a particle in the reservoir. The reservoir 
is also taken to be sufficiently large that it can be treated in the thermodynamic limit and is in an equilibrium with temperature $T$ and chemical potential $\mu$ for times $t<t_{0}$. At $t_{0}$ it is brought into contact with the trap by means of a tunnel Hamiltonian

$$
\hat{H}^{\mathrm{int}}=\frac{1}{\sqrt{V}} \sum_{\mathbf{n}} \sum_{\mathbf{k}}\left(t_{\mathbf{n}}(\mathbf{k}) \hat{\psi}_{\mathbf{n}}(t) \hat{\psi}_{\mathbf{k}}^{\dagger}(t)+t_{\mathbf{n}}^{*}(\mathbf{k}) \hat{\psi}_{\mathbf{k}}(t) \hat{\psi}_{\mathbf{n}}^{\dagger}(t)\right),
$$

with complex tumneling matrix elements $t_{\mathbf{n}}(\mathbf{k})$ that for simplicity are assimed to be almost constant for momenta $\hbar k$ smaller that a cutoff $h k_{c}$ but to vanish rapidly for momenta larger than this ultraviolet cutoff. Moreover, we consider here only the low-temperature regime in which the thermal de Broglie wavelength $\Lambda_{\mathrm{th}}=\left(2 \pi h^{2} / m k_{B} T\right)^{1 / 2}$ of the particles obeys $\Lambda_{\mathrm{th}} \gg 1 / k_{\mathrm{c}}$, since this is the most appropriate limit for realistic atomic gases.

To study the evolution of the combined system for times $t \geq t_{0}$ we thus have to deal with the action

$$
\begin{aligned}
S\left[\psi^{*}, \psi ; \psi \psi_{\mathrm{R}}^{*}, \psi_{\mathrm{R}}\right]= & \sum_{\mathbf{n}} \int_{\mathcal{C}^{\infty}} \mathrm{d} t \psi_{\mathbf{n}}^{*}(t)\left(i \hbar \frac{\partial}{\partial t}-\epsilon_{\mathbf{n}}+\mu\right) \psi_{\mathbf{n}}(t) \\
& +\sum_{\mathbf{k}} \int_{\mathcal{C}^{\infty}} \mathrm{d} t \psi_{\mathbf{k}}^{*}(t)\left(i \hbar \frac{\partial}{\partial t}-\epsilon(\mathbf{k})+\mu\right) \psi_{\mathbf{k}}(t) \\
& -\frac{1}{\sqrt{V}} \sum_{\mathbf{n}} \sum_{\mathbf{k}} \int_{\mathcal{C}^{\infty}} \mathrm{d} t\left(t_{\mathbf{n}}(\mathbf{k}) \psi_{\mathbf{n}}(t) \psi_{\mathbf{k}}^{*}(t)\right. \\
& \left.+t_{\mathbf{n}}^{*}(\mathbf{k}) \psi_{\mathbf{k}}(t) \psi_{\mathbf{n}}^{*}(t)\right)
\end{aligned}
$$

if we measure all energies relative to the chemical potential and also introduce the complex field $\psi_{\mathrm{R}}(\mathrm{x}, t)=\sum_{\mathbf{k}} \psi_{\mathbf{k}}(t) \chi_{\mathbf{k}}(\mathbf{x})$ for the degrees of freedom of the reservoir. However, we are only interested in the evolution of the Bose gas in the trap and therefore only in the time-ordered correlation functions of this part of the system. The corresponding generating functional

$$
\begin{aligned}
Z\left[J, J^{*}\right]= & \int \mathrm{d}\left[\psi^{*}\right] \mathrm{d}[\psi] \int \mathrm{d}\left[\psi_{\mathrm{R}}^{*}\right] \mathrm{d}\left[\psi_{\mathrm{R}}\right] \exp \left\{\frac{i}{\hbar} S\left[\psi^{*}, \psi ; \psi_{\mathrm{R}}^{*}, \psi_{\mathrm{R}}\right]\right\} \\
& \times \exp \left\{i \int_{\mathcal{C}^{\infty}} \mathrm{d} t \int \mathrm{d} \mathbf{x}\left(\psi(\mathbf{x}, t) J^{*}(\mathbf{x}, t)+J(\mathbf{x}, t) \psi^{*}(\mathbf{x}, t)\right)\right\}
\end{aligned}
$$

is of the same form as the functional integral in equation (3.56), but now with an effective action that is defined by

$$
\exp \left\{\frac{i}{\hbar} S^{\mathrm{eff}}\left[\psi^{*}, \psi\right]\right\} \equiv \int \mathrm{d}\left[\psi_{\mathrm{R}}^{*}\right] \mathrm{d}\left[\psi_{\mathrm{R}}\right] \exp \left\{\frac{i}{\hbar} S\left[\psi^{*}, \psi ; \psi_{\mathrm{R}}^{*}, \psi_{\mathrm{R}}\right]\right\} .
$$


Hence, our next task is to integrate out the field $\psi_{\mathrm{R}}(\mathrm{x}, t)$, which can be done exactly because it only requires the integration of a ganssian.

To familiarize ourselves with the Schwinger-Keldysh formalism, we perform the gaussian integration here in some detail. In principle, this can of course be done explicitly by making use of the fact that the initial density matrix $\hat{\rho}_{\mathrm{R}}\left(t_{0}\right)$ of the reservoir can be expanded in the projection operators on the coherent states by using the coefficients

$$
\rho_{\mathrm{R}}\left[\left|\phi_{\mathrm{R}}\right|^{2} ; t_{0}\right]=\prod_{\mathbf{k}} \frac{1}{N(\mathbf{k})} \mathrm{e}^{-\left|\phi_{\mathbf{k}}\right|^{2} / N(\mathbf{k})} .
$$

with $N(\mathbf{k})=1 /\left(\mathrm{e}^{3(\epsilon(\mathbf{k})-\mu)}-1\right)$ the appropriate Bose distribution function and $\beta=1 / k_{B} T$. However, in practice it is much more easy to use a different procedure. It is based on the observation that if we introduce the $\delta$ function on the Schwinger-Keldysh contour defined by $\int_{\mathcal{C} x} \mathrm{~d} t^{\prime} \delta\left(t, t^{\prime}\right)=1$ and the Green's function $G\left(\mathbf{k} ; t, t^{\prime}\right)$ obeying

$$
\left(i \hbar \frac{\partial}{\partial t}-\epsilon(\mathbf{k})+\mu\right) G\left(\mathbf{k} ; t, t^{\prime}\right)=\hbar \delta\left(t, t^{\prime}\right)
$$

the action $S\left[\psi^{*}, \psi ; \psi_{\mathrm{R}}^{*}, \psi_{\mathrm{R}}\right]$ can be written as a complete square, or more precisely as the sum of two squares $S_{1}\left[\psi^{*}, \psi\right]$ and $S_{2}\left[\psi^{*}, \psi ; \psi_{\mathrm{R}}^{*}, \psi_{\mathrm{R}}\right]$ that are given by

$$
\begin{aligned}
S_{1}\left[\psi^{*}, \psi\right]= & \sum_{\mathbf{n}} \int_{\mathcal{C} x} \mathrm{~d} t \psi_{\mathbf{n}}^{*}(t)\left(i \hbar \frac{\partial}{\partial t}-\epsilon_{\mathbf{n}}+\mu\right) \psi_{\mathbf{n}}(t) \\
& -\frac{1}{\hbar V} \sum_{\mathbf{n} \cdot \mathbf{n}^{\prime}} \sum_{\mathbf{k}} \int_{\mathcal{C}^{\infty}} \mathrm{d} t \int_{\mathcal{C} x} \mathrm{~d} t^{\prime} \psi_{\mathbf{n}}^{*}(t) t_{\mathbf{n}}^{*}(\mathbf{k}) G\left(\mathbf{k} ; t, t^{\prime}\right) t_{\mathbf{n}^{\prime}}(\mathbf{k}) \psi_{\mathbf{n}^{\prime}}\left(t^{\prime}\right)
\end{aligned}
$$

and

$$
\begin{aligned}
S_{2}\left[\psi^{*}, \psi ; \psi_{\mathrm{R}}^{*}, \psi_{\mathrm{R}}\right]= & \sum_{\mathbf{k}} \int_{\mathcal{C}^{x}} \mathrm{~d} t\left(\psi_{\mathbf{k}}^{*}(t)\right. \\
& \left.-\frac{1}{\hbar \sqrt{V}} \sum_{\mathbf{n}} \int_{\mathcal{C}^{\infty}} \mathrm{d} t^{\prime} t_{\mathbf{n}}^{*}(\mathbf{k}) \psi_{\mathbf{n}}^{*}\left(t^{\prime}\right) G\left(\mathbf{k} ; t^{\prime}, t\right)\right) \\
& \times\left(i \hbar \frac{\partial}{\partial t}-\epsilon(\mathbf{k})+\mu\right) \\
& \times\left(\psi_{\mathbf{k}}(t)-\frac{1}{\hbar \sqrt{V}} \sum_{\mathbf{n}} \int_{\mathcal{C}^{\infty}} \mathrm{d} t^{\prime} G\left(\mathbf{k} ; t, t^{\prime}\right) \psi_{\mathbf{n}}\left(t^{\prime}\right) t_{\mathbf{n}}(\mathbf{k})\right),
\end{aligned}
$$

respectively. Since the first term is independent of the field $\psi_{\mathrm{R}}(\mathrm{x}, t)$, we only need to evaluate $\int \mathrm{d}\left[\psi_{\mathrm{R}}^{*}\right] \mathrm{d}\left[\psi_{\mathrm{R}}\right] \exp \left(i S_{2}\left[\psi^{*}, \psi ; \psi_{\mathrm{R}}^{*}, \psi_{\mathrm{R}}\right] / \hbar\right)$. Performing 
a shift in the integration variables, we however see that this functional integral is equal to

$$
\begin{array}{r}
\int \mathrm{d}\left[\psi_{\mathrm{R}}^{*}\right] \mathrm{d}\left[\psi_{\mathrm{R}}\right] \exp \left\{\frac{i}{\hbar} \sum_{\mathbf{k}} \int_{\mathcal{C}^{\infty}} \mathrm{d} t \psi_{\mathbf{k}}^{*}(t)\left(i \hbar \frac{\partial}{\partial t}-\epsilon(\mathbf{k})+\mu\right) \psi_{\mathbf{k}}(t)\right\} \\
=\operatorname{Tr}\left[\rho_{\mathrm{R}}\left(t_{0}\right)\right]=1 .
\end{array}
$$

As a result the effective action $S^{\mathrm{eff}}\left[\psi^{*}, \psi\right]$ is just equal to $S_{1}\left[\psi^{*}, \psi\right]$, which can be slightly rewritten to read

$$
\begin{aligned}
S^{\mathrm{eff}}\left[\psi^{*}, \psi\right]= & \sum_{\mathbf{n} \cdot \mathbf{n}^{\prime}} \int_{\mathcal{C}^{x}} \mathrm{~d} t \int_{\mathcal{C}^{\infty}} \mathrm{d} t^{\prime} \\
& \times \psi_{\mathbf{n}}^{*}(t)\left\{\left(i \hbar \frac{\partial}{\partial t}-\epsilon_{\mathbf{n}}+\mu\right) \delta_{\mathbf{n} \cdot \mathbf{n}^{\prime}} \delta\left(t, t^{\prime}\right)-\hbar \Sigma_{\mathbf{n} \cdot \mathbf{n}^{\prime}}\left(t, t^{\prime}\right)\right\} \psi_{\mathbf{n}^{\prime}}\left(t^{\prime}\right),
\end{aligned}
$$

with a selfonergy $\Sigma_{\mathbf{n}, \mathbf{n}^{\prime}}\left(t, t^{\prime}\right)$ obeying

$$
\hbar \Sigma_{\mathbf{n}, \mathbf{n}^{\prime}}\left(t, t^{\prime}\right)=\frac{1}{\hbar V} \sum_{\mathbf{k}} t_{\mathbf{n}}^{*}(\mathbf{k}) G\left(\mathbf{k} ; t, t^{\prime}\right) t_{\mathbf{n}^{\prime}}(\mathbf{k}) .
$$

This is our first example of an effective action describing the nonequilibrium dynamies of a Bose gas. Before we can study its consequences we clearly first need to determine the Green's function $G\left(\mathbf{k} ; t, t^{\prime}\right)$ in terms of which the selfencrgy is expressed. Although we know that this Green's function fulfills equation (3.64), we camnot directly solve this equation because we do not know the appropriate boundary condition at $t=t^{\prime}$. To calculate $G\left(\mathrm{k}: t, t^{\prime}\right)$ we therefore have to proceed differently. It is however clear from equation (3.64) that $G\left(\mathbf{k} ; t, t^{\prime}\right)$ is a property of the reservoir and we thus expect that it can somehow be related to a time-ordered correlation function of this reservior. To see that explicitly we consider again the generating functional of these correlation functions, i.e.,

$$
\begin{aligned}
Z_{\mathrm{R}}\left[J, J^{*}\right]= & \int \mathrm{d}\left[\psi_{\mathrm{R}}^{*}\right] \mathrm{d}\left[\psi_{\mathrm{R}}\right] \\
& \times \exp \left\{\frac{i}{\hbar} \sum_{\mathbf{k}} \int_{\mathcal{C}^{\infty}} \mathrm{d} t \psi_{\mathbf{k}}^{*}(t)\left(i \hbar \frac{\partial}{\partial t}-\epsilon(\mathbf{k})+\mu\right) \psi_{\mathbf{k}}(t)\right\} \\
& \times \exp \left\{i \sum_{\mathbf{k}} \int_{\mathcal{C}^{\infty}} \mathrm{d} t\left(\psi_{\mathbf{k}}(t) J_{\mathbf{k}}^{*}(t)+J_{\mathbf{k}}(t) \psi_{\mathbf{k}}^{*}(t)\right)\right\} .
\end{aligned}
$$

It is again a gaussian integral and can thus be evaluated in the same manner as before. The result is now

$$
Z_{\mathrm{R}}\left[J, J^{*}\right]=\exp \left\{-i \sum_{\mathbf{k}} \int_{\mathcal{C}^{\infty}} \mathrm{d} t \int_{\mathcal{C} \infty} \mathrm{d} t^{\prime} J_{\mathbf{k}}^{*}(t) G\left(\mathbf{k}: t, t^{\prime}\right) J_{\mathbf{k}}\left(t^{\prime}\right)\right\},
$$


which shows by means of equation (3.58) that

$$
i G\left(\mathbf{k} ; t, t^{\prime}\right)=\operatorname{Tr}\left[\hat{\rho}_{\mathrm{R}}\left(t_{0}\right) T_{\mathcal{C} \times}\left(\hat{\psi}_{\mathbf{k}}(t) \hat{\psi}_{\mathbf{k}}^{\dagger}\left(t^{\prime}\right)\right)\right] .
$$

Note first of all that this is indeed consistent with equation (3.64), because the right-hand side obeys

$$
\begin{aligned}
& i \hbar \frac{\partial}{\partial t} \operatorname{Tr}\left[\hat{\rho}_{\mathrm{R}}\left(t_{0}\right) T_{\mathcal{C}}\right.\left.\left(\hat{\psi}_{\mathbf{k}}(t) \hat{\psi}_{\mathbf{k}}^{\dagger}\left(t^{\prime}\right)\right)\right]=i \hbar \delta\left(t, t^{\prime}\right) \operatorname{Tr}\left[\hat{\rho}_{\mathrm{R}}\left(t_{0}\right)\left[\hat{\psi}_{\mathbf{k}}(t), \hat{\psi}_{\mathbf{k}}^{\dagger}(t)\right]\right] \\
&+\operatorname{Tr}\left[\hat{\rho}_{\mathrm{R}}\left(t_{0}\right) T_{\mathcal{C}}\left(i \hbar \frac{\partial}{\partial t} \hat{\psi}_{\mathbf{k}}(t) \hat{\psi}_{\mathbf{k}}^{\dagger}\left(t^{\prime}\right)\right)\right] \\
&=i \hbar \delta\left(t, t^{\prime}\right)+(\epsilon(\mathbf{k})-\mu) \operatorname{Tr}\left[\hat{\rho}_{\mathrm{R}}\left(t_{0}\right) T_{\mathcal{C}}\left(\hat{\psi}_{\mathbf{k}}(t) \hat{\psi}_{\mathbf{k}}^{\dagger}\left(t^{\prime}\right)\right)\right]
\end{aligned}
$$

in the operator formalism. Moreover, from this identification we see that the desired solution fulfilling the appropriate boundary conditions is apparently

$$
G\left(\mathbf{k} ; t, t^{\prime}\right)=-i \mathrm{e}^{-i(\epsilon(\mathbf{k})-\mu)\left(t-t^{\prime}\right) / \hbar}\left\{\Theta\left(t, t^{\prime}\right)(1+N(\mathbf{k}))+\Theta\left(t^{\prime}, t\right) N(\mathbf{k})\right\} .
$$

The specific dependence on the backward and forward branches of the Schwinger-Keldysh contour is thus solely determined by the Heaviside function $\Theta\left(t, t^{\prime}\right)$. As a result it is convenient to decompose the Green's function into its analytic pieces $G^{>}\left(\mathbf{k} ; t-t^{\prime}\right)$ and $G^{<}\left(\mathbf{k} ; t-t^{\prime}\right)$ [83] by means of

$$
G\left(\mathbf{k} ; t, t^{\prime}\right)=\Theta\left(t, t^{\prime}\right) G^{>}\left(\mathbf{k} ; t-t^{\prime}\right)+\Theta\left(t^{\prime}, t\right) G^{<}\left(\mathbf{k} ; t-t^{\prime}\right) .
$$

Due to the fact that we are always dealing with time-ordered correlation functions, such a decomposition turns out to be a generic feature of all the functions on the Schwinger-Keldysh contour that we will encounter in the following [84]. For a general function $F\left(t, t^{\prime}\right)$, it is however also possible to have $\delta$-function singularities. If that happens the correct decomposition is

$$
F\left(t, t^{\prime}\right)=F^{\delta}(t) \delta\left(t, t^{\prime}\right)+\Theta\left(t, t^{\prime}\right) F^{>}\left(t, t^{\prime}\right)+\Theta\left(t^{\prime}, t\right) F^{<}\left(t, t^{\prime}\right) .
$$

This more general decomposition is not required here, but will be needed in Section 3.4 when we determine the effective interaction between two atoms in a gas.

Having obtained the Green's function of the reservoir, we can now return to our discussion of the effective action $S^{\operatorname{eff}}\left[\psi^{*}, \psi\right]$ for the Bose gas in the trap. Although we have chosen to derive in equation (3.68) the effective action for the generating functional $Z\left[J, J^{*}\right]$, it is straightforward to show that the effective action for the probability distribution $P\left[\phi^{*}, \phi ; t\right]$ is obtained by only replacing the contour $\mathcal{C}^{\infty}$ by $\mathcal{C}^{t}$. In the following we therefore no longer always specify all the boundary conditions on the time 
integration, if the precise details of this integration are not important and the discussion applies equally well to both cases. Keeping this in mind, we now again perform the transformation $\psi_{\mathbf{n}}\left(t_{ \pm}\right)=\phi_{\mathbf{n}}(t) \pm \xi_{\mathbf{n}}(t) / 2$ to explicitly separate the (semi)classical dynamics from the effect of fluctuations. It leads in first instance to [85]

$$
\begin{aligned}
S^{\mathrm{eff}}\left[\phi^{*}, \phi ; \xi^{*}, \xi\right]= & \sum_{\mathbf{n}} \int_{t_{0}} \mathrm{~d} t \phi_{\mathbf{n}}^{*}(t)\left(i \hbar \frac{\partial}{\partial t}-\epsilon_{\mathbf{n}}+\mu\right) \xi_{\mathbf{n}}(t) \\
& +\sum_{\mathbf{n}} \int_{t_{0}} \mathrm{~d} t \xi_{\mathbf{n}}^{*}(t)\left(i \hbar \frac{\partial}{\partial t}-\epsilon_{\mathbf{n}}+\mu\right) \phi_{\mathbf{n}}(t) \\
& -\sum_{\mathbf{n} \cdot \mathbf{n}^{\prime}} \int_{\left.t_{0}\right)} \mathrm{d} t \int_{t_{0}} \mathrm{~d} t^{\prime} \phi_{\mathbf{n}}^{*}(t) h \Sigma_{\mathbf{n} \cdot \mathbf{n}^{\prime}}^{(-)}\left(t-t^{\prime}\right) \xi_{\mathbf{n}^{\prime}}\left(t^{\prime}\right) \\
& -\sum_{\mathbf{n} \cdot \mathbf{n}^{\prime}} \int_{t_{0}} \mathrm{~d} t \int_{t_{0}} \mathrm{~d} t^{\prime} \xi_{\mathbf{n}}^{*}(t) \hbar \Sigma_{\mathbf{n} \cdot \mathbf{n}^{\prime}}^{(+)}\left(t-t^{\prime}\right) \phi_{\mathbf{n}^{\prime}}\left(t^{\prime}\right) \\
& -\frac{1}{2} \sum_{\mathbf{n} \cdot \mathbf{n}^{\prime}} \int_{t_{0}} \mathrm{~d} t \int_{t_{0}} \mathrm{~d} t^{\prime} \xi_{\mathbf{n}}^{*}(t) \hbar \Sigma_{\mathbf{n} \cdot \mathbf{n}^{\prime}}^{K}\left(t-t^{\prime}\right) \xi_{\mathbf{n}^{\prime}}\left(t^{\prime}\right),
\end{aligned}
$$

where we introduced the retarded and advanced components of the selfenergy

$$
\Sigma_{\mathbf{n}, \mathbf{n}^{\prime}}^{( \pm)}\left(t-t^{\prime}\right)= \pm \Theta\left( \pm\left(t-t^{\prime}\right)\right)\left(\Sigma_{\mathbf{n} \cdot \mathbf{n}^{\prime}}^{>}\left(t-t^{\prime}\right)-\Sigma_{\mathbf{n}, \mathbf{n}^{\prime}}^{<}\left(t-t^{\prime}\right)\right)
$$

that affect the terms in the action that are linear in $\xi_{\mathbf{n}}^{*}(t)$ and $\xi_{\mathbf{n}}(t)$. respectively, and also the Keldysh component

$$
\Sigma_{\mathbf{n}, \mathbf{n}^{\prime}}^{\mathrm{K}}\left(t-t^{\prime}\right)=\left(\Sigma_{\mathbf{n}, \mathbf{n}^{\prime}}^{>}\left(t-t^{\prime}\right)+\Sigma_{\mathbf{n}, \mathbf{n}^{\prime}}^{<}\left(t-t^{\prime}\right)\right)
$$

that is associated with the part quadratic in the fluctuations.

The physical content of these various components of the selfenergy is understood most clearly if we now apply again the beautiful functionalintegral procedure that is due to Hubbard and Stratonovich. The basic idea is to write the factor

$$
\exp \left\{-\frac{i}{2} \sum_{\mathbf{n}, \mathbf{n}^{\prime}} \int_{t_{0}} \mathrm{~d} t \int_{t_{0}} \mathrm{~d} t^{\prime} \xi_{\mathbf{n}}^{*}(t) \Sigma_{\mathbf{n}, \mathbf{n}^{\prime}}^{\mathrm{K}}\left(t-t^{\prime}\right) \xi_{\mathbf{n}^{\prime}}\left(t^{\prime}\right)\right\}
$$

in the integrant of $\int \mathrm{d}\left[\phi^{*}\right] \mathrm{d}[\phi] \int \mathrm{d}\left[\xi^{*}\right] \mathrm{d}[\xi] \exp \left(i S^{\mathrm{eff}}\left[\phi^{*}, \phi ; \xi^{*}, \xi\right] / \hbar\right)$ as a gaussian integral over a complex field $\eta(\mathbf{x}, t)$. It is equivalent, but in practice more convenient, to just multiply the integrant by a factor 1 that is 
written as a gaussian integral $\int \mathrm{d}\left[\eta^{*}\right] \mathrm{d}[\eta] \exp \left(i S^{\operatorname{eff}}\left[\eta^{*}, \eta\right] / \hbar\right)$ with

$$
\begin{aligned}
S^{\mathrm{eff}}\left[\eta^{*} \cdot \eta\right]= & \frac{1}{2} \sum_{\mathbf{n} \cdot \mathbf{n}^{\prime}} \int_{t_{0}} \mathrm{~d} t \int_{t_{0}} \mathrm{~d} t^{\prime}\left(2 \eta_{\mathbf{n}}^{*}(t)\right. \\
& \left.-\sum_{\mathbf{n}^{\prime \prime}} \int_{t_{0}} \mathrm{~d} t^{\prime \prime} \xi_{\mathbf{n}^{\prime \prime}}\left(t^{\prime \prime}\right) \hbar \Sigma_{\mathbf{n}^{\prime \prime} \cdot \mathbf{n}}^{\mathrm{K}}\left(t^{\prime \prime}-t\right)\right) \\
& \times\left(\hbar \Sigma^{\mathrm{K}}\right)_{\mathbf{n}, \mathbf{n}^{\prime}}^{-1}\left(t-t^{\prime}\right)\left(2 \eta_{\mathbf{n}^{\prime}}\left(t^{\prime}\right)\right. \\
& \left.-\sum_{\mathbf{n}^{\prime \prime}} \int_{t_{0}} \mathrm{~d} t^{\prime \prime} \hbar \Sigma_{\mathbf{n}^{\prime} \cdot \mathbf{n}^{\prime \prime}}^{\mathrm{K}}\left(t^{\prime}-t^{\prime \prime}\right) \xi_{\mathbf{n}^{\prime \prime}}\left(t^{\prime \prime}\right)\right)
\end{aligned}
$$

a complete square. Adding this to $S^{\mathrm{eff}}\left[\phi^{*}, \phi ; \xi^{*}, \xi\right]$ the total effective action becomes

$$
\begin{aligned}
S^{\mathrm{eff}}\left[\phi^{*}, \phi: \xi^{*}, \xi ; \eta^{*}, \eta\right]= & \sum_{\mathbf{n}, \mathbf{n}^{\prime}} \int_{t_{0}} \mathrm{~d} t \int_{t_{0}} \mathrm{~d} t^{\prime} \phi_{\mathbf{n}}^{*}(t)\left\{\left(i \hbar \frac{\partial}{\partial t}-\epsilon_{\mathbf{n}} \quad(3.81\right.\right. \\
& \left.+\mu) \delta_{\mathbf{n}, \mathbf{n}^{\prime}} \delta\left(t-t^{\prime}\right)-\hbar \Sigma_{\mathbf{n} \cdot \mathbf{n}^{\prime}}^{(-)}\left(t-t^{\prime}\right)\right\} \xi_{\mathbf{n}^{\prime}}\left(t^{\prime}\right) \\
& +\sum_{\mathbf{n}, \mathbf{n}^{\prime}} \int_{t_{0}} \mathrm{~d} t \int_{t_{0}} \mathrm{~d} t^{\prime} \xi_{\mathbf{n}}^{*}(t)\left\{\left(i \hbar \frac{\partial}{\partial t}-\epsilon_{\mathbf{n}}\right.\right. \\
& \left.+\mu) \delta_{\mathbf{n} \cdot \mathbf{n}^{\prime}} \delta\left(t-t^{\prime}\right)-\hbar \Sigma_{\mathbf{n}, \mathbf{n}^{\prime}}^{(t)}\left(t-t^{\prime}\right)\right\} \phi_{\mathbf{n}^{\prime}}\left(t^{\prime}\right) \\
& -\sum_{n} \int_{t_{0}} \mathrm{~d} t\left(\eta_{n}^{*}(t) \xi_{n}(t)+\xi_{n}^{*}(t) \eta_{n}(t)\right) \\
& +2 \sum_{\mathbf{n}, \mathbf{n}^{\prime}} \int_{t_{0}} \mathrm{~d} t \int_{t_{0}} \mathrm{~d} t^{\prime} \eta_{\mathbf{n}}^{*}(t)\left(\hbar \Sigma^{K}\right)_{\mathbf{n}, \mathbf{n}^{\prime}}^{-1}\left(t-t^{\prime}\right) \eta_{\mathbf{n}^{\prime}}\left(t^{\prime}\right)
\end{aligned}
$$

and is thus linear in $\xi_{\mathbf{n}}(t)$ and $\xi_{\mathbf{n}}^{*}(t)$. Integrating over these fluctuations we conclude from this action that the field $\phi(\mathbf{x}, t)$ is constraint to obey the Langevin equations [86]

$$
i \hbar \frac{\partial}{\partial t} \phi_{\mathbf{n}}(t)=\left(\epsilon_{\mathbf{n}}-\mu\right) \phi_{\mathbf{n}}(t)+\sum_{\mathbf{n}^{\prime}} \int_{t_{0}}^{\infty} \mathrm{d} t^{\prime} \hbar \Sigma_{\mathbf{n}, \mathbf{n}^{\prime}}^{(+)}\left(t-t^{\prime}\right) \phi_{\mathbf{n}^{\prime}}\left(t^{\prime}\right)+\eta_{\mathbf{n}}(t)
$$

and

$$
-i \hbar \frac{\partial}{\partial t} \phi_{\mathbf{n}}^{*}(t)=\left(\epsilon_{\mathbf{n}}-\mu\right) \phi_{\mathbf{n}}^{*}(t)+\sum_{\mathbf{n}^{\prime}} \int_{t_{0}}^{\infty} \mathrm{d} t^{\prime} \phi_{\mathbf{n}^{\prime}}^{*}\left(t^{\prime}\right) \hbar \Sigma_{\mathbf{n}^{\prime}, \mathbf{n}}^{(-)}\left(t^{\prime}-t\right)+\eta_{\mathbf{n}}^{*}(t)
$$


with gaussian noise terms $\eta_{\mathbf{n}}(t)$ and $\eta_{\mathbf{n}}^{*}(t)$ that from the last term in the right-hand side of equation (3.81) are seen to have the time correlations

$$
\begin{aligned}
\left\langle\eta_{\mathbf{n}}^{*}(t) \eta_{\mathbf{n}^{\prime}}\left(t^{\prime}\right)\right\rangle & =\frac{i h^{2}}{2} \Sigma_{\mathbf{n} \cdot \mathbf{n}^{\prime}}^{K}\left(t-t^{\prime}\right) \\
& =\frac{1}{2} \int \frac{\mathrm{d} \mathbf{k}}{(2 \pi)^{3}}(1+2 N(\mathbf{k})) t_{\mathbf{n}}^{*}(\mathbf{k}) \mathrm{e}^{-i(\epsilon(\mathbf{k})-\mu)\left(t-t^{\prime}\right) / \hbar} t_{\mathbf{n}^{\prime}}(\mathbf{k})
\end{aligned}
$$

in the thermodynamic limit. Moreover, in that limit the retarded selfenergy is equal to

$$
h \Sigma_{\mathbf{n}, \mathbf{n}^{\prime}}^{(t)}\left(t-t^{\prime}\right)=-\Theta\left(t-t^{\prime}\right) \frac{i}{\hbar} \int \frac{\mathrm{d} \mathbf{k}}{(2 \pi)^{3}} t_{\mathbf{n}}^{*}(\mathbf{k}) \mathrm{e}^{-i(\epsilon(\mathbf{k})-\mu)\left(t-t^{\prime}\right) / \hbar} t_{\mathbf{n}^{\prime}}(\mathbf{k})
$$

and the advanced selfenergy can be found from the relation $\Sigma_{\mathbf{n}^{\prime}, \mathbf{n}}^{(-)}\left(t^{\prime}-t\right)=$ $\left(\Sigma_{\mathbf{n} \cdot \mathbf{n}^{\prime}}^{(t)}\left(t-t^{\prime}\right)\right)^{*}$. Note that the Heaviside functions in these retarded and advanced solfenergies are precisely such that equations (3.82) and (3.83) are causal, i.e., the evolution of $\phi(\mathrm{x}, t)$ and $\phi^{*}(\mathrm{x}, t)$ depends only on their value at previous times. This is clearly a sensible result and explains why these components of the full selfenergy enter into the terms in the action that are linear in the fluctuations.

To understand why the Keldysh component enters into the terms that are quadratic in the fluctuations is more complicated and is related to the famous fluctuation-dissipation theorem [83,86,87]. Physically, the fluctuationdissipation theorem ensures in our case that, due to the interactions with the reservoir, the gas in the trap now relaxes towards thermal equilibrium in the limit $t \rightarrow \infty$. Let us therefore study this limit in somewhat more detail. However, before we do so we would like to mention for completeness that if we apply the above Hubbard-Stratonovich transformation to the functional integral for the probability distribution $P\left[\phi^{*}, \phi ; t\right]$, we immediately find that analogous to equation $(3.47)$

$$
\begin{aligned}
P\left[\phi^{*}, \phi ; t\right]= & \int \mathrm{d}\left[\eta^{*}\right] \mathrm{d}[\eta] P\left[\eta^{*}, \eta\right] \int \mathrm{d}\left[\phi_{0}^{*}\right] \mathrm{d}\left[\phi_{0}\right] P\left[\left|\phi_{0}\right|^{2} ; t_{0}\right] \\
& \times \prod_{\mathbf{n}} \delta\left(\left|\phi_{\mathbf{n}}-\phi_{\mathbf{n}}^{\mathrm{cl}}(t)\right|^{2}\right),
\end{aligned}
$$

where $\phi_{\mathbf{n}}^{\mathrm{cl}}(t)$ now obeys the Langevin equation in equation (3.82) for a particular realization of the noise $\eta_{\mathbf{n}}(t)$ and with the initial condition $\phi_{\mathbf{n}}^{\mathrm{cl}}\left(t_{0}\right)=$ $\phi_{0 ; \mathbf{n}}$. Furthermore, the probability distribution for each realization of the 
noise is given by

$$
P\left[\eta^{*}, \eta\right]=\exp \left\{\frac{2 i}{\hbar} \sum_{\mathbf{n} \cdot \mathbf{n}^{\prime}} \int_{t_{01}}^{t} \mathrm{~d} t^{\prime} \int_{t_{t \prime}}^{t} \mathrm{~d} t^{\prime \prime} \eta_{\mathbf{n}}^{*}\left(t^{\prime}\right)\left(\hbar \Sigma^{\mathrm{K}}\right)_{\mathbf{n} \cdot \mathbf{n}^{\prime}}^{-1}\left(t^{\prime}-t^{\prime \prime}\right) \eta_{\mathbf{n}^{\prime}}\left(t^{\prime \prime}\right)\right\} .
$$

This result shows explicitly that, as expected, the Fokker-Planck equation associated with the Langevin equations in equations (3.82) and (3.83) is in fact the desired Fokker-Planck equation for the probability distribution $P\left[\phi^{*} . \phi: t\right]$.

After this slight digression, we now return to the long-time behaviour of the gas. In the long-time limit two important simplifications occur. Looking at the expresions for the selfenergies, we see that their width as a function of the time difference $t-t^{\prime}$ is at most of order $\mathcal{O}\left(\hbar / k_{B} T\right)$ in the low-temperature regime of interest where the thermal de Broglie wavclength of the particles obeys $\Lambda_{t h_{1}} \gg 1 / k_{\mathrm{c}}$. If therefore $t \gg t_{0}+\mathcal{O}\left(\hbar / k_{B} T\right)$ we are allowed to take the limit $t_{0} \rightarrow-\infty$. Taking this limit means physically that we neglect the initial transients that are due to the precise way in which the contact between the trap and the reservoir is made, and focus on the "universal" dynamics that is independent of these details. In addition, at long times the dynamics of the gas is expected to be sufficiently slow that we can neglect the memory effects altogether. Finally, we consider here first the case of a reservoir that is so weakly coupled to the gas in the trap that we can treat the coupling with second order perturbation theory. As a result we can also neglect the nondiagonal elements of the selfenergies. In total we are then allowed to put (but see below)

$$
\Sigma_{\mathbf{n} \cdot \mathbf{n}^{\prime}}^{( \pm) \cdot K}\left(t-t^{\prime}\right) \simeq \Sigma_{\mathbf{n}, \mathbf{n}}^{( \pm) \cdot K}\left(\epsilon_{\mathbf{n}}-\mu\right) \delta_{\mathbf{n}, \mathbf{n}^{\prime}} \delta\left(t-t^{\prime}\right) \equiv \Sigma_{\mathbf{n}}^{( \pm) \cdot K} \delta_{\mathbf{n}, \mathbf{n}^{\prime}} \delta\left(t-t^{\prime}\right) .
$$

where the Fourier transform of the selfenergies is defined by

$$
\Sigma_{\mathbf{n}, \mathbf{n}^{\prime}}^{( \pm), K}\left(t-t^{\prime}\right)=\int \mathrm{d} \epsilon \Sigma_{\mathbf{n}, \mathbf{n}^{\prime}}^{( \pm)} \cdot \mathrm{K}(\epsilon) \frac{\mathrm{e}^{-i \epsilon\left(t-t^{\prime}\right) / \hbar}}{2 \pi \hbar}
$$

Note that this implies in general that $\Sigma_{\mathbf{n}^{\prime}, \mathbf{n}}^{(-)}(\epsilon)=\left(\Sigma_{\mathbf{n}, \mathbf{n}^{\prime}}^{(+)}(\epsilon)\right)^{*}$, and in particular therefore that $\Sigma_{\mathbf{n}}^{(-)}=\left(\Sigma_{\mathbf{n}}^{(+)}\right)^{*}$.

With these simplifications our Langevin equations become

$$
i \hbar \frac{\partial}{\partial t} \phi_{\mathbf{n}}(t)=\left(\epsilon_{\mathbf{n}}+\hbar \Sigma_{\mathbf{n}}^{(+)}-\mu\right) \phi_{\mathbf{n}}(t)+\eta_{\mathbf{n}}(t)
$$


and just the complex conjugate equation for $\phi_{\mathbf{n}}^{*}(t)$. The retarded selfenergy in this equation is given by

$$
\hbar \Sigma_{\mathbf{n}}^{(+)}=\int \frac{\mathrm{d} \mathbf{k}}{(2 \pi)^{3}} t_{\mathbf{n}}^{*}(\mathbf{k}) \frac{1}{\epsilon_{\mathbf{n}}^{+}-\epsilon(\mathbf{k})} t_{\mathbf{n}}(\mathbf{k}),
$$

with $\epsilon_{\mathbf{n}}^{+}$the usual notation for the limiting procedure $\epsilon_{\mathbf{n}}+i 0$. It clearly has real and imaginary parts that we denote by $S_{\mathbf{n}}$ and $-R_{\mathbf{n}}$, respectively. Denoting the Cauchy principle value part of an integral by $\mathcal{P}$, they obey

$$
S_{\mathbf{n}}=\int \frac{\mathrm{d} \mathbf{k}}{(2 \pi)^{3}} t_{\mathbf{n}}^{*}(\mathbf{k}) \frac{\mathcal{P}}{\epsilon_{\mathbf{n}}-\epsilon(\mathbf{k})} t_{\mathbf{n}}(\mathbf{k})
$$

and

$$
R_{\mathbf{n}}=\pi \int \frac{\mathrm{d} \mathbf{k}}{(2 \pi)^{3}} \delta\left(\epsilon_{\mathbf{n}}-\epsilon(\mathbf{k})\right)\left|t_{\mathbf{n}}(\mathbf{k})\right|^{2} .
$$

The interpretation of these results is quite obvious if we consider the average of the Langevin equation, i.e.,

$$
i \hbar \frac{\partial}{\partial t}\left\langle\phi_{\mathbf{n}}\right\rangle(t)=\left(\epsilon_{\mathbf{n}}+S_{\mathbf{n}}-i R_{\mathbf{n}}-\mu\right)\left\langle\phi_{\mathbf{n}}\right\rangle(t) .
$$

which is solved by

$$
\left\langle\phi_{\mathbf{n}}\right\rangle(t)=\left\langle\phi_{\mathbf{n}}\right\rangle(0) \mathrm{e}^{-i\left(\epsilon_{\mathbf{n}}+S_{\mathbf{n}}-\mu\right) t / \hbar} \mathrm{e}^{-R_{\mathbf{n}} t / \hbar} .
$$

Hence the real part of the retarded selfenergy $S_{\mathbf{n}}$ represents the shift in the energy of state $\chi_{\mathbf{n}}(\mathrm{x})$ due to the coupling with the reservoir. Indeed, equation (3.92) agrees precisely with the value of this shift in second order perturbation theory. Moreover, the fact that $\left|\left\langle\phi_{\mathbf{n}}\right\rangle(t)\right|^{2}=\left|\left\langle\phi_{\mathbf{n}}\right\rangle(0)\right|^{2} \mathrm{e}^{-2 R_{\mathbf{n}} t / \hbar}$ shows that the average rate of decay $\Gamma_{\mathbf{n}}$ of the state $\chi_{\mathbf{n}}(\mathrm{x})$ is equal to $2 R_{\mathbf{n}} / \hbar$, which, together with equation (3.93), exactly reproduces Fermi's Golden Rule.

From the equation of motion for the average $\left\langle\phi_{\mathbf{n}}\right\rangle(t)$ we can also conclude that the right-hand side of the Fokker-Planck equation contains the "streaming" terms

$$
\begin{aligned}
-\left(\sum _ { \mathbf { n } } \frac { \partial } { \partial \phi _ { \mathbf { n } } } \left(\epsilon_{\mathbf{n}}+\hbar \Sigma_{\mathbf{n}}^{(+)}\right.\right. & \left.-\mu) \phi_{\mathbf{n}}\right) P\left[\phi^{*}, \phi ; t\right] \\
& +\left(\sum_{\mathbf{n}} \frac{\partial}{\partial \phi_{\mathbf{n}}^{*}}\left(\epsilon_{\mathbf{n}}+\hbar \Sigma_{\mathbf{n}}^{(-)}-\mu\right) \phi_{\mathbf{n}}^{*}\right) P\left[\phi^{*}, \phi ; t\right] .
\end{aligned}
$$

However, there must now also be a "diffusion" term due to the fact that the average $\left\langle\left|\phi_{\mathbf{n}}\right|^{2}\right\rangle(t)$ is no longer independent of time since the interactions 
with the reservoir can lead to changes in the occupation numbers $N_{\mathbf{n}}(t)$. To

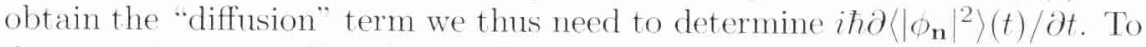
do so we first formally solve the Langevin equation by

$$
\phi_{\mathbf{n}}(t)=\mathrm{e}^{-i\left(\epsilon_{\mathbf{n}}+h \Sigma_{\mathbf{n}}^{(+)}-\mu\right) t / \hbar}\left\{\phi_{\mathbf{n}}(0)-\frac{i}{\hbar} \int_{0}^{t} \mathrm{~d} t^{\prime} \eta\left(t^{\prime}\right) \mathrm{e}^{i\left(\epsilon_{\mathbf{n}}+h \Sigma_{\mathbf{n}}^{(+)}-\mu\right) t^{\prime} / h}\right\} .
$$

Multiplying this with the complex conjugate expression and taking the average, we obtain first of all

$$
\left\langle\left|\phi_{\mathbf{n}}\right|^{2}\right\rangle(t)=\mathrm{e}^{-2 R_{\mathbf{n}} t / \hbar}\left\{\left\langle\left|\phi_{\mathbf{n}}\right|^{2}\right\rangle(0)+\frac{i}{2} \Sigma_{\mathbf{n}}^{\mathrm{K}} \int_{0}^{t} \mathrm{~d} t^{\prime} \mathrm{e}^{2 R_{\mathbf{n}} t^{\prime} / h}\right\},
$$

which shows that

$$
i \hbar \frac{\partial}{\partial t}\left\langle\left|\phi_{\mathbf{n}}\right|^{2}\right\rangle(t)=-2 i R_{\mathbf{n}}\left\langle\left|\phi_{\mathbf{n}}\right|^{2}\right\rangle(t)-\frac{1}{2} \hbar \Sigma_{\mathbf{n}}^{K} .
$$

Moreover, the Keldysh component of the selfenergy is given by

$$
\hbar_{i} \Sigma_{\mathbf{n}}^{\mathrm{K}}=-2 \pi i \int \frac{\mathrm{d} \mathbf{k}}{(2 \pi)^{3}}(1+2 N(\mathbf{k})) \delta\left(\epsilon_{\mathbf{n}}-\epsilon(\mathbf{k})\right)\left|t_{\mathbf{n}}(\mathbf{k})\right|^{2}
$$

and therefore obeys

$$
h \sum_{\mathbf{n}}^{\mathrm{K}}=-2 i\left(1+2 N_{\mathbf{n}}\right) R_{\mathbf{n}}
$$

with $N_{\mathrm{n}}=\left(\mathrm{e}^{\beta\left(\epsilon_{\mathrm{n}}-\mu\right)}-1\right)^{-1}$ the Bose distribution function. This is in fact the fluctuation-dissipation theorem, because it relates the strength of the fluctuations determined by $\hbar \Sigma_{\mathbf{n}}^{K}$, to the amount of dissipation that is given by $R_{\mathbf{n}}$. As mentioned previously, the fluctuation-dissipation theorem ensures that the gas relaxes to thermal equilibrium. This we can already see from equation (3.98), because substitution of the fluctuation-rissipation theorem leads to

$$
i \hbar \frac{\partial}{\partial t}\left\langle\left|\phi_{\mathbf{n}}\right|^{2}\right\rangle(t)=-2 i R_{\mathbf{n}}\left\langle\left|\phi_{\mathbf{n}}\right|^{2}\right\rangle(t)+i R_{\mathbf{n}}\left(1+2 N_{\mathbf{n}}\right),
$$

which tells us that in equilibrium $\left\langle\left|\phi_{\mathbf{n}}\right|^{2}\right\rangle=N_{\mathbf{n}}+1 / 2$. In fact, we have argued that in general $\left\langle\left|\phi_{\mathbf{n}}\right|^{2}\right\rangle(t)=N_{\mathbf{n}}(t)+1 / 2$. Substituting this identity in equation (3.101) and realizing that $\Gamma_{\mathbf{n}} N_{\mathbf{n}}=\Gamma_{\mathbf{n}} N(\mathbf{k})$ due to the cnergyconserving $\delta$ function in $\Gamma_{\mathbf{n}}$, we indeed obtain the correct rate equation for the average occupation numbers

$$
\begin{aligned}
\frac{\partial}{\partial t} N_{\mathbf{n}}(t) & =-\Gamma_{\mathbf{n}} N_{\mathbf{n}}(t)+\Gamma_{\mathbf{n}} N_{\mathbf{n}} \\
& =-\Gamma_{\mathbf{n}} N_{\mathbf{n}}(t)(1+N(\mathbf{k}))+\Gamma_{\mathbf{n}}\left(1+N_{\mathbf{n}}(t)\right) N(\mathbf{k}),
\end{aligned}
$$


that might justly be called the quantum Boltzmann equation for the gas, because the right-hand side contains precisely the rates for scattering into and out of the reservoir.

Furthermore, equation (3.98) shows that the "diffusion" term in the Fokker-Planck equation is

$$
-\left(\frac{1}{2} \sum_{\mathbf{n}} \frac{\partial^{2}}{\partial \phi_{\mathbf{n}}^{*} \partial \phi_{\mathbf{n}}} \hbar \Sigma_{\mathbf{n}}^{K}\right) P\left[\phi^{*}, \phi ; t\right],
$$

because the first term in the right-hand side of equation (3.98) is also present without the noise and is therefore already accounted for in the "streaming" terms. In total we have thus arrived at

$$
\begin{aligned}
i \hbar \frac{\partial}{\partial t} P\left[\phi^{*}, \phi ; t\right]= & -\left(\sum_{\mathbf{n}} \frac{\partial}{\partial \phi_{\mathbf{n}}}\left(\epsilon_{\mathbf{n}}+\hbar \Sigma_{\mathbf{n}}^{(+)}-\mu\right) \phi_{\mathbf{n}}\right) P\left[\phi^{*}, \phi ; t\right] \\
& +\left(\sum_{\mathbf{n}} \frac{\partial}{\partial \phi_{\mathbf{n}}^{*}}\left(\epsilon_{\mathbf{n}}+\hbar \Sigma_{\mathbf{n}}^{(-)}-\mu\right) \phi_{\mathbf{n}}^{*}\right) P\left[\phi^{*}, \phi ; t\right] \\
& -\left(\frac{1}{2} \sum_{\mathbf{n}} \frac{\partial^{2}}{\partial \phi_{\mathbf{n}}^{*} \partial \phi_{\mathbf{n}}} \hbar \Sigma_{\mathbf{n}}^{K}\right) P\left[\phi^{*}, \phi ; t\right]
\end{aligned}
$$

Using again the fluctuation-dissipation theorem, it is not difficult to show that the stationary solution of this Fokker-Planck equation is

$$
P\left[\phi^{*}, \phi ; \infty\right]=\prod_{\mathbf{n}} \frac{1}{N_{\mathbf{n}}+1 / 2} \exp \left\{-\frac{1}{N_{\mathbf{n}}+1 / 2}\left|\phi_{\mathbf{n}}\right|^{2}\right\} .
$$

Although this appears to be quite reasonable, it is important to note that this result is not fully consistent because the "streaming" terms in equation (3.103) show that the energies of the states $\chi_{\mathbf{n}}(\mathbf{x})$ are shifted and equal to $\epsilon_{\mathbf{n}}+S_{\mathbf{n}}$. We therefore expect that the equilibrium occupation numbers are equal to the Bose distribution evaluated at those energies and not at the unshifted values $\epsilon_{\mathbf{n}}$. The reason for this inconsistency is, of course, that we have taken $\Sigma_{\mathbf{n}}^{( \pm) . K}$ equal to $\Sigma_{\mathbf{n}, \mathbf{n}}^{( \pm)}\left(\epsilon_{\mathbf{n}}-\mu\right)$, which was only justified on the basis of second-order perturbation theory. We now, however, see that to obtain a fully consistent theory we must take instead $\Sigma_{\mathbf{n}}^{( \pm), K} \equiv \Sigma_{\mathbf{n}, \mathbf{n}}^{( \pm)}\left(\epsilon_{\mathbf{n}}+S_{\mathbf{n}}-\mu\right)$. This implies that to obtain the retarded selfenergy, we first have to solve for the real part

$$
S_{\mathbf{n}}=\int \frac{\mathrm{d} \mathbf{k}}{(2 \pi)^{3}} t_{\mathbf{n}}^{*}(\mathbf{k}) \frac{\mathcal{P}}{\epsilon_{\mathbf{n}}+S_{\mathbf{n}}-\epsilon(\mathbf{k})} t_{\mathbf{n}}(\mathbf{k})
$$

and then substitute this into

$$
R_{\mathbf{n}}=\pi \int \frac{\mathrm{d} \mathbf{k}}{(2 \pi)^{3}} \delta\left(\epsilon_{\mathbf{n}}+S_{\mathbf{n}}-\epsilon(\mathbf{k})\right)\left|t_{\mathbf{n}}(\mathbf{k})\right|^{2}
$$


to obtain the imaginary part. The fluctuation-dissipation theorem then again reads $\hbar \Sigma_{\mathbf{n}}^{\mathrm{K}}=-2 i\left(1+2 N_{\mathbf{n}}\right) R_{\mathbf{n}}$ but now with the equilibrium distribution $N_{\mathbf{n}}=\left(\mathrm{e}^{\beta\left(\epsilon_{\mathrm{n}}+S_{\mathrm{n}}-\mu\right)}-1\right)^{-1}$. As a consequence the quantum Boltzmann equation and the stationary state of the Fokker-Planck equation will now also contain these correct equilibrium occupation numbers [88].

Having arrived at the Fokker-Planck equation of our Caldeira-Leggettlike toy model, we have now demonstrated the use of essentially all the theoretical tools that are required for a discussion of a weakly interacting Bose gas. Before we start with this discussion, however, we want to make a final remark about the effect of the nondiagonal elements of the selfenergies. Physically, including these nondiagonal elements accounts for the change in the wave functions $\chi_{\mathbf{n}}(\mathbf{x})$ due to the interaction with the reservoir. It can clearly be neglected if the coupling with the reservoir is sufficiently weak. or more precisely if $\left|\hbar \Sigma_{\mathbf{n}^{\prime}, \mathbf{n}}^{(+)}\left(\epsilon_{\mathbf{n}}+S_{\mathbf{n}}-\mu\right)\right|$ is much smaller than the energy splitting $\left|\epsilon_{\mathbf{n}^{\prime}}-\epsilon_{\mathbf{n}}\right|$ between the states in the trap. For a quantum dot in solidstate phyisics [89] this is often the case and for that reason we might call our toy model a bosonic quantum dot. However, in magnetically trapped atomic gases the average interaction energy between the atoms is already slightly below the Bose-Einstein condensation critical temperature larger than the typical energy splitting and the nondiagonal elements of the selfenergies are very important. Such a strong-coupling situation can, of course, also be treated in our Caldeira-Leggett like model. The main difference is that we need to expand our various fields not in the eigenstates of the trapping potential $\chi_{\mathbf{n}}(\mathbf{x})$ but in the eigenstates $\chi_{\mathbf{n}}^{\prime}(\mathbf{x})$ of the nonlocal Schrödinger equation

$$
\begin{aligned}
\epsilon_{\mathbf{n}}^{\prime} \chi_{\mathbf{n}}^{\prime}(\mathrm{x})= & \left(-\frac{\hbar^{2} \nabla^{2}}{2 m}+V^{\mathrm{ex}}(\mathrm{x})\right) \chi_{\mathbf{n}}^{\prime}(\mathrm{x}) \\
& +\int \mathrm{d} \mathrm{x}^{\prime} \operatorname{Re}\left[\hbar \Sigma^{(+)}\left(\mathrm{x}, \mathrm{x}^{\prime} ; \epsilon_{\mathbf{n}}^{\prime}-\mu\right)\right] \chi_{\mathbf{n}}^{\prime}\left(\mathrm{x}^{\prime}\right)
\end{aligned}
$$

where $\epsilon_{\mathbf{n}}^{\prime}$ are the new eigenvalues and the retarded selfenergie in coordinate space is $\hbar \Sigma^{(+)}\left(\mathbf{x}, \mathbf{x}^{\prime} ; \epsilon\right)=\sum_{\mathbf{n}, \mathbf{n}^{\prime}} \chi_{\mathbf{n}}(\mathbf{x}) \hbar \Sigma_{\mathbf{n}, \mathbf{n}^{\prime}}^{(+)}(\epsilon) \chi_{\mathbf{n}^{\prime}}^{*}\left(\mathbf{x}^{\prime}\right)$. In this new basis the nondiagonal elements of the selfenergies can now be neglected and we find essentially the same results as before. We only need to replace $\epsilon_{\mathbf{n}}+S_{\mathbf{n}}$ by $\epsilon_{\mathbf{n}}^{\prime}$ and $t_{\mathbf{n}}(\mathbf{k})$ by $\sum_{\mathbf{n}^{\prime}}\left(\int \mathrm{d} \mathbf{x} \chi_{\mathbf{n}}^{\prime}\left(\mathbf{x}^{\prime}\right) \chi_{\mathbf{n}^{\prime}}^{*}(\mathbf{x})\right) t_{\mathbf{n}^{\prime}}(\mathbf{k})$. Neglecting the nondiagonal elements in this basis only requires that the real part of the retarded selfenergy is much larger that its imaginary part, which is always fulfilled in our case because $k_{\mathrm{c}} \Lambda_{\mathrm{th}} \gg 1$.

Summarizing, the coherent and incoherent dynamics of the gas in the trap is for our toy model quite generally solved by

$$
\left\langle\phi_{\mathbf{n}}\right\rangle(t)=\left\langle\phi_{\mathbf{n}}\right\rangle(0) \mathrm{e}^{-i\left(\epsilon_{\mathbf{n}}^{\prime}-\mu\right) t / \hbar} \mathrm{e}^{-\Gamma_{\mathbf{n}} t / 2}
$$


and

$$
N_{\mathbf{n}}(t)=N_{\mathbf{n}}(0) \mathrm{e}^{-\Gamma_{\mathbf{n}} t}+N_{\mathbf{n}}\left(1-\mathrm{e}^{-\Gamma_{\mathbf{n}} t}\right),
$$

respectively. In the limit $t \rightarrow \infty$, the average of the annihilation operators $\left\langle\phi_{\mathbf{n}}\right\rangle(t)$ thus always vanishes but the average occupation numbers $N_{\mathbf{n}}(t)$ relax to the equilibrium distribution $N_{n}=\left(\mathrm{e}^{\beta\left(\epsilon_{\mathrm{n}}^{\prime}-\mu\right)}-1\right)^{-1}$. Although this appears to be an immediately obvious result, its importance stems from the fact that it is also true if we tume the potential energy bias $\Delta V^{\text {ex }}$ such that at low temperatures the groundstate $\chi_{\mathbf{0}}^{\prime}(\mathrm{x})$ acquires a macroscopic occupation, i.e., $N_{0} \gg 1$. The gas therefore never shows a spontaneous breaking of the $U(1)$ symmetry, in agrecment with the notion that we are esscntially dealing with an ideal Bose gas in the grand-canonical ensemble $[90]$. The reason of the abscuse of the spontaneous symmetry breaking can also be nuderstood from onr stationary solution of the Fokker-Planck equation in equation (3.104), which shows that the probability distribution for $\left|\phi_{0}\right|$ is proportional to the Boltzmann factor $\mathrm{e}^{-\beta\left(\epsilon_{0}^{\prime}-\mu\right)\left|\phi_{0}\right|^{2}}$ in the degenerate regime of interest. Honce, the corresponding Landau free energy $F_{\mathrm{L}}\left(\left|\phi_{0}\right|\right)=\left(\epsilon_{0}^{\prime}-\mu\right)\left|\phi_{0}\right|^{2}$ never slows an instability towards the formation of a nonzero average of $\left|\phi_{0}\right|$ due to the fact that $\epsilon_{0}^{\prime}-\mu$ can never become less then zero. Once we introduce interactions between the atoms in the gas, this picture fully changes.

\subsection{Condensate formation}

We are now in a position to discuss an interacting Bose gas, because in an interacting Bose gas, the gas is roughly speaking its own themal reservoir. The Fokker-Planck equation therefore turns out to be quite similar to the one presented in equation (3.103). To be more precise, however, we should mention that we aim in this section to describe the formation of a condensate in an interacting Bose gas under the conditions that have recently been realized in experiments with atomic rubidium, lithium, sodium and hydrogen gases. In these experiments the gas is cooled by means of evaporative cooling. Numerical solutions of the Boltzmann equation have shown that during evaporative cooling the energy distribution function is well described by an equilibrium distribution with time dependent temperature $T(t)$ and chemical potential $\mu(t)$, that is truncated at high energies due to the evaporation of the highest energetic atoms from the trap [91]. Moreover, in the experiments of interest the densities just above the critical temperature are essentially always such that the gas is in the weak-coupling limit, which implies in this context that the average interaction energy per atom is always much less than the energy splitting of the one-particle states in the harmonic trapping potential. 


\subsubsection{Weak-coupling limit}

For a trapped atomic gas the appropriate one-particle states are the eigenstates $\chi_{\mathbf{n}}(\mathrm{x})$ of the external potential $V^{\mathrm{ex}}(\mathrm{x})$. As a result the Fokker-Planck equation for the interacting gas will now quite gencrally be given by

$$
\begin{aligned}
& i \hbar \frac{\partial}{\partial t} P\left[\phi^{*} \cdot \phi: t\right]=-\sum_{\mathbf{n} \cdot \mathbf{n}^{\prime}} \frac{\partial}{\partial \phi_{\mathbf{n}}}\left\{\left(\left(\epsilon_{\mathbf{n}}-\mu(t)\right) \delta_{\mathbf{n} \cdot \mathbf{n}^{\prime}}+\hbar \Sigma_{\mathbf{n} \cdot \mathbf{n}^{\prime}}^{(+)}\right) \phi_{\mathbf{n}^{\prime}}\right. \\
& \left.+\sum_{\mathbf{m} \cdot \mathbf{m}^{\prime}} V_{\mathbf{n} \cdot \mathbf{m}: \mathbf{n}^{\prime} \cdot \mathbf{m}^{\prime}}^{(+)} \phi_{\mathbf{m}}^{*} \phi_{\mathbf{m}^{\prime}} \phi_{\mathbf{n}^{\prime}}\right\} P\left[\phi^{*} \cdot \phi ; t\right] \\
& +\sum_{\mathbf{n}, \mathbf{n}^{\prime}} \frac{\partial}{\partial \phi_{\mathbf{n}}^{*}}\left\{\left(\left(\epsilon_{\mathbf{n}}-\mu(t)\right) \delta_{\mathbf{n}, \mathbf{n}^{\prime}}+h \Sigma_{\mathbf{n}^{\prime} \cdot \mathbf{n}}^{(-)}\right) \phi_{\mathbf{n}^{\prime}}^{*}\right. \\
& \left.+\sum_{\mathbf{m} \cdot \mathbf{m}^{\prime}} V_{\mathbf{n}^{\prime} \cdot \mathbf{m}^{\prime} ; \mathbf{n} \cdot \mathbf{m}}^{(-)} \phi_{\mathbf{n}^{\prime}}^{*} \phi_{\mathbf{m}^{\prime}}^{*} \phi_{\mathbf{m}}\right\} P\left[\phi^{*}, \phi: t\right] \\
& -\frac{1}{2} \sum_{\mathbf{n}, \mathbf{n}^{\prime}} \frac{\partial^{2}}{\partial \phi_{\mathbf{n}} \partial \phi_{\mathbf{n}^{\prime}}^{*}}\left\{h \Sigma_{n, \mathbf{n}^{\prime}}^{K}\right. \\
& \left.+\sum_{\mathbf{m}, \mathbf{m}^{\prime}} V_{\mathbf{n}, \mathbf{m} ; \mathbf{n}^{\prime}, \mathbf{m}^{\prime}}^{\mathrm{K}} \phi_{\mathbf{m}}^{*} \phi_{\mathbf{m}^{\prime}}\right\} P\left[\phi^{*}, \phi: t\right] .
\end{aligned}
$$

For this equation to be useful, however, we need to determine the various selfenergies and interactions. To do so, let us for simplicity first consider the normal state of the gas which is usually in the weak-coupling limit $\left|\hbar \Sigma_{\mathbf{n}, \mathbf{n}^{\prime}}^{(+)}\right| \ll\left|\epsilon_{\mathbf{n}}-\epsilon_{\mathbf{n}^{\prime}}\right|$ for the experiments with atomic alkali gases that are of interest here. In this regime we can neglect the nondiagonal elements of the selfenergies and also the nonlinear terms in the right-hand side of equation (3.110). The Fokker-Planck equation then reduces to

$$
\begin{aligned}
i \hbar \frac{\partial}{\partial t} P\left[\phi^{*}, \phi ; t\right]= & -\sum_{\mathbf{n}} \frac{\partial}{\partial \phi_{\mathbf{n}}}\left(\epsilon_{\mathbf{n}}+\hbar \Sigma_{\mathbf{n}}^{(+)}-\mu(t)\right) \phi_{\mathbf{n}} P\left[\phi^{*}, \phi ; t\right] \\
& +\sum_{\mathbf{n}} \frac{\partial}{\partial \phi_{\mathbf{n}}^{*}}\left(\epsilon_{\mathbf{n}}+\hbar \Sigma_{\mathbf{n}}^{(-)}-\mu(t)\right) \phi_{\mathbf{n}}^{*} P\left[\phi^{*}, \phi ; t\right] \\
& -\frac{1}{2} \sum_{\mathbf{n}} \frac{\partial^{2}}{\partial \phi_{\mathbf{n}}^{*} \partial \phi_{\mathbf{n}}} \hbar \Sigma_{\mathbf{n}}^{K} P\left[\phi^{*}, \phi ; t\right],
\end{aligned}
$$

with $\hbar \Sigma_{\mathbf{n}}^{( \pm) \cdot K}=\hbar \Sigma_{\mathbf{n}, \mathbf{n}}^{( \pm), K}\left(\epsilon_{\mathbf{n}}^{\prime}-\mu\right)$ and $\epsilon_{\mathbf{n}}^{\prime}=\epsilon_{\mathbf{n}}+S_{\mathbf{n}}$. This equation looks identical to the one we derived for our Caldeira-Leggett toy model but describes in fact quite different physics because now the selfenergies are not 
duc to an interaction with a reservoir but due to the interactions between the atoms themselves. This is, however, completely hidden in the selfenergies as we show now explicitly. Performing the same manipulations as in Sections 2.5 and 2.8.2, we find in the many-body T-matrix approximation first of all that

$$
\begin{aligned}
\hbar \Sigma_{\mathbf{n}}^{(+)}=\left(\hbar \Sigma_{\mathbf{n}}^{(-)}\right)^{*}= & 2 \sum_{\mathbf{m}} V_{\mathbf{n}, \mathbf{m} ; \mathbf{n}, \mathbf{m}}^{(+)}\left(\epsilon_{\mathbf{n}}^{\prime}+\epsilon_{\mathbf{m}}^{\prime}-2 \mu\right) N_{\mathbf{m}} \\
& +2 i \sum_{\mathbf{m}} \int \frac{d \epsilon}{2 \pi \hbar} V_{\mathbf{n} \cdot \mathbf{m}: \mathbf{n} \cdot \mathbf{m}}^{<}(\epsilon) \frac{\hbar}{\epsilon^{-}-\left(\epsilon_{\mathbf{n}}^{\prime}+\epsilon_{\mathbf{m}}^{\prime}-2 \mu\right)}
\end{aligned}
$$

and similarly that

$$
\begin{aligned}
\hbar \Sigma_{\mathbf{n}}^{\mathrm{K}}= & 2 \sum_{\mathbf{m}} V_{\mathbf{n}, \mathbf{m}: \mathbf{n}, \mathbf{m}}^{\mathrm{K}}\left(\epsilon_{\mathbf{n}}^{\prime}+\epsilon_{\mathbf{m}}^{\prime}-2 \mu\right) N_{\mathbf{m}} \\
& +2 \sum_{\mathbf{m}} V_{\mathbf{n}, \mathbf{m}: \mathbf{n} \cdot \mathbf{m}}^{<}\left(\epsilon_{\mathbf{n}}^{\prime}+\epsilon_{\mathbf{m}}^{\prime}-2 \mu\right)\left(1+2 N_{\mathbf{m}}\right) .
\end{aligned}
$$

Our next task is therefore the evaluation of the various many-body T-matrix clements $V_{\mathbf{n}, \mathbf{m} ; \mathbf{n}^{\prime} \cdot \mathbf{m}^{\prime}}^{(+),<. K}(\epsilon) \equiv\left(T_{\mathbf{n}, \mathbf{m} ; \mathbf{n}^{\prime}, \mathbf{m}^{\prime}}^{(+),<. K}(\epsilon)+T_{\mathbf{m} \cdot \mathbf{n}: \mathbf{n}^{\prime}, \mathbf{m}^{\prime}}^{(+)}(\epsilon)\right) / 2$ that occur in these expressions, which is casily carried out with our experience of the homogeneous gas and by noting that the Lippmam-Schwinger equation is in fact an operator equation that only needs to be expressed in a new basis. In terms of the appropriate matrix elements of the interatomic interaction, i.e..

$$
V_{\mathbf{n} \cdot \mathbf{m}: \mathbf{n}^{\prime} \cdot \mathbf{m}^{\prime}}=\int \mathrm{d} \mathbf{x} \int \mathrm{d} \mathbf{x}^{\prime} \chi_{\mathbf{n}}^{*}(\mathbf{x}) \chi_{\mathbf{m}}^{*}\left(\mathrm{x}^{\prime}\right) V\left(\mathbf{x}-\mathbf{x}^{\prime}\right) \chi_{\mathbf{n}^{\prime}}(\mathbf{x}) \chi_{\mathbf{m}^{\prime}}\left(\mathrm{x}^{\prime}\right) .
$$

the retarded and advanced components of the many-body $\mathrm{T}$ matrix thus obey the Bethe-Salpeter equation [92]

$$
\begin{aligned}
T_{\mathbf{n}, \mathbf{m} ; \mathbf{n}^{\prime}, \mathbf{m}^{\prime}}^{(\epsilon)=} & V_{\mathbf{n} \cdot \mathbf{m} ; \mathbf{n}^{\prime}, \mathbf{m}^{\prime}} \\
& +\sum_{\mathbf{n}^{\prime \prime}, \mathbf{m}^{\prime \prime}} V_{\mathbf{n}, \mathbf{m} ; \mathbf{n}^{\prime \prime}, \mathbf{m}^{\prime \prime}} \frac{1+N_{\mathbf{n}^{\prime \prime}}+N_{\mathbf{m}^{\prime \prime}}}{\epsilon^{ \pm}-\left(\epsilon_{\mathbf{n}^{\prime \prime}}^{\prime}+\epsilon_{\mathbf{m}^{\prime \prime}}^{\prime}-2 \mu\right)} T_{\mathbf{n}^{\prime \prime}, \mathbf{m}^{\prime \prime} ; \mathbf{n}^{\prime} \cdot \mathbf{m}^{\prime}}^{( \pm)}(\epsilon)
\end{aligned}
$$

and the optical theorem

$$
\begin{aligned}
T_{\mathbf{n}, \mathbf{m} ; \mathbf{n}^{\prime}, \mathbf{m}^{\prime}}^{(+)}(\epsilon)- & T_{\mathbf{n}, \mathbf{m} ; \mathbf{n}^{\prime}, \mathbf{m}^{\prime}}^{(-)}(\epsilon)=-2 \pi i \sum_{\mathbf{n}^{\prime \prime}, \mathbf{m}^{\prime \prime}} \delta\left(\epsilon-\left(\epsilon_{\mathbf{n}^{\prime \prime}}^{\prime}+\epsilon_{\mathbf{m}^{\prime \prime}}^{\prime}-2 \mu\right)\right) \\
& \times T_{\mathbf{n}, \mathbf{m} ; \mathbf{n}^{\prime \prime}, \mathbf{m}^{\prime \prime}}^{(+)}(\epsilon)\left(1+N_{\mathbf{n}^{\prime \prime}}+N_{\mathbf{m}^{\prime \prime}}\right) T_{\mathbf{n}^{\prime \prime}, \mathbf{m}^{\prime \prime} ; \mathbf{n}^{\prime}, \mathbf{m}^{\prime}}^{(-)}(\epsilon)
\end{aligned}
$$

Finally, we also need

$$
\begin{aligned}
T_{\mathbf{n}, \mathbf{m} ; \mathbf{n}^{\prime}, \mathbf{m}^{\prime}}^{<}(\epsilon)= & -2 \pi i \sum_{\mathbf{n}^{\prime \prime}, \mathbf{m}^{\prime \prime}} \delta\left(\epsilon-\left(\epsilon_{\mathbf{n}^{\prime \prime}}^{\prime}+\epsilon_{\mathbf{m}^{\prime \prime}}^{\prime}-2 \mu\right)\right) \\
& \times T_{\mathbf{n}, \mathbf{m} ; \mathbf{n}^{\prime \prime}, \mathbf{m}^{\prime \prime}}^{(+)}(\epsilon) N_{\mathbf{n}^{\prime \prime}} N_{\mathbf{m}^{\prime \prime}} T_{\mathbf{n}^{\prime \prime}, \mathbf{m}^{\prime \prime} ; \mathbf{n}^{\prime}, \mathbf{m}^{\prime}}^{(-)}(\epsilon)
\end{aligned}
$$


and the Keldysh component

$$
\begin{aligned}
& T_{\mathbf{n} \cdot \mathbf{m}: \mathbf{n}^{\prime} \cdot \mathbf{m}^{\prime}}^{\mathrm{K}}(\epsilon)=-2 \pi i \sum_{\mathbf{n}^{\prime \prime} \cdot \mathbf{m}^{\prime \prime}} \delta\left(\epsilon-\left(\epsilon_{\mathbf{n}^{\prime \prime}}^{\prime}+\epsilon_{\mathbf{m}^{\prime \prime}}^{\prime}-2 \mu\right)\right) \\
& \quad \times T_{\mathbf{n} \cdot \mathbf{m}: \mathbf{n}^{\prime \prime} \cdot \mathbf{m}^{\prime \prime}}^{(+)}(\epsilon)\left(1+N_{\mathbf{n}^{\prime \prime}}+N_{\mathbf{m}^{\prime \prime}}+2 N_{\mathbf{n}^{\prime \prime}} N_{\mathbf{m}^{\prime \prime}}\right) T_{\mathbf{n}^{\prime \prime}, \mathbf{m}^{\prime \prime}: \mathbf{n}^{\prime} \cdot \mathbf{m}^{\prime}}^{(-)}(\epsilon) .
\end{aligned}
$$

Notice that the appearance of a $\delta$ function in the last three equations in practice never leads to any problems, because in the experiments with trapped atomic gases the number of particles is always so large that the critical temperature of the gas is much larger than the energy splitting of the trap and the discrete sum over states is well approximated by an integral over a continuous spectrum.

Making use of the above relations we find that for the states with thermal energies the renormalized energy is given by the pseudopotential result

$$
\epsilon_{\mathbf{n}}^{\prime}(t)=\epsilon_{\mathbf{n}}+\frac{8 \pi a h^{2}}{m} \int \mathrm{d} \mathbf{x} \chi_{\mathbf{n}}^{*}(\mathrm{x}) n(\mathrm{x}, t) \lambda_{\mathbf{n}}(\mathrm{x}) .
$$

with $n(\mathrm{x} . t)$ the density profile of the gas cloud. For the states near the one-particle grom state there is, however, a correction given by

$$
-2 \sum_{\mathbf{m}} \sum_{\mathbf{n}^{\prime \prime} \cdot \mathbf{m}^{\prime \prime}}\left|V_{\mathbf{n}, \mathbf{m} ; \mathbf{n}^{\prime \prime} \cdot \mathbf{m}^{\prime \prime}}^{(+)}(t)\right|^{2} N_{\mathbf{n}^{\prime \prime}}(t) N_{\mathbf{m}^{\prime \prime}}(t) \frac{\mathcal{P}}{\epsilon_{\mathbf{n}}^{\prime}(t)+\epsilon_{\mathbf{m}}^{\prime}(t)-\epsilon_{\mathbf{n}^{\prime \prime}}^{\prime}(t)-\epsilon_{\mathbf{m}^{\prime \prime}}^{\prime}(t)}
$$

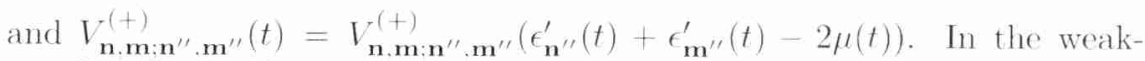
coupling limit, the renormalization of the one-particle energies plays by definition not such an important role and we are much more interested in the imaginary part of the retarded and advanced selfenergies. They are given by

$$
\begin{aligned}
R_{\mathbf{n}}(t)= & 2 \pi \sum_{\mathbf{m}} \sum_{\mathbf{n}^{\prime \prime} \cdot \mathbf{m}^{\prime \prime}} \delta\left(\epsilon_{\mathbf{n}^{\prime \prime}}^{\prime}(t)+\epsilon_{\mathbf{m}^{\prime \prime}}^{\prime}(t)-\epsilon_{\mathbf{n}}^{\prime}(t)-\epsilon_{\mathbf{m}}^{\prime}(t)\right) \\
& \times\left|V_{\mathbf{n}, \mathbf{m}: \mathbf{n}^{\prime \prime} \cdot \mathbf{m}^{\prime \prime}}^{(+)}(t)\right|^{2}\left[\left(1+N_{\mathbf{n}^{\prime \prime}}(t)+N_{\mathbf{m}^{\prime \prime}}(t)\right) N_{\mathbf{m}}(t)-N_{\mathbf{n}^{\prime \prime}}(t) N_{\mathbf{m}^{\prime \prime}}(t)\right] .
\end{aligned}
$$

Furthermore, the Keldysh component of the selfenergies equals

$$
\begin{aligned}
\hbar \sum_{\mathbf{n}}^{K}(t)= & -4 \pi \sum_{\mathbf{m}} \sum_{\mathbf{n}^{\prime \prime}, \mathbf{m}^{\prime \prime}} \delta\left(\epsilon_{\mathbf{n}^{\prime \prime}}^{\prime}(t)+\epsilon_{\mathbf{m}^{\prime \prime}}^{\prime}(t)-\epsilon_{\mathbf{n}}^{\prime}(t)-\epsilon_{\mathbf{m}}^{\prime}(t)\right) \\
& \times\left|V_{\mathbf{n}, \mathbf{m} ; \mathbf{n}^{\prime \prime}, \mathbf{m}^{\prime \prime}}^{(+)}(t)\right|^{2}\left[\left(1+N_{\mathbf{n}^{\prime \prime}}(t)+N_{\mathbf{m}^{\prime \prime}}(t)\right) N_{\mathbf{m}}(t)\right. \\
& \left.+N_{\mathbf{n}^{\prime \prime}}(t) N_{\mathbf{m}^{\prime \prime}}(t)\left(1+2 N_{\mathbf{m}}(t)\right)\right] .
\end{aligned}
$$

As a result the quantum Boltzmann equation for the gas becomes

$$
\frac{\partial}{\partial t} N_{\mathbf{n}}(t)=-\Gamma_{\mathbf{n}}^{o n t}(t) N_{\mathbf{n}}(t)+\Gamma_{\mathbf{n}}^{i n}(t)\left(1+N_{\mathbf{n}}(t)\right),
$$


with the rate to scatter out of the state $\chi_{\mathbf{n}}(\mathbf{x})$ given by

$$
\begin{aligned}
\Gamma_{\mathbf{n}}^{o n t}(t)= & \frac{4 \pi}{\hbar} \sum_{\mathbf{m}} \sum_{\mathbf{n}^{\prime \prime} \cdot \mathbf{m}^{\prime \prime}} \delta\left(\epsilon_{\mathbf{n}^{\prime \prime}}^{\prime}(t)+\epsilon_{\mathbf{m}^{\prime \prime}}^{\prime}(t)-\epsilon_{\mathbf{n}}^{\prime}(t)-\epsilon_{\mathbf{m}}^{\prime}(t)\right) \\
& \times\left|V_{\mathbf{n} \cdot \mathbf{m}: \mathbf{n}^{\prime \prime}, \mathbf{m}^{\prime \prime}}^{(+)}(t)\right|^{2}\left(1+N_{\mathbf{n}^{\prime \prime}}(t)\right)\left(1+N_{\mathbf{m}^{\prime \prime}}(t)\right) N_{\mathbf{m}}(t)
\end{aligned}
$$

and the rate to scatter into this state by

$$
\begin{aligned}
\Gamma_{\mathbf{n}}^{i n}(t)= & \frac{4 \pi}{\hbar} \sum_{\mathbf{m}} \sum_{\mathbf{n}^{\prime \prime}, \mathbf{m}^{\prime \prime}} \delta\left(\epsilon_{\mathbf{n}^{\prime \prime}}^{\prime}(t)+\epsilon_{\mathbf{m}^{\prime \prime}}^{\prime}(t)-\epsilon_{\mathbf{n}}^{\prime}(t)-\epsilon_{\mathbf{m}}^{\prime}(t)\right) \\
& \times\left|V_{\mathbf{n} \cdot \mathbf{m}: \mathbf{n}^{\prime \prime} \cdot \mathbf{m}^{\prime \prime}}^{(t)}(t)\right|^{2} N_{\mathbf{n}^{\prime \prime}}(t) N_{\mathbf{m}^{\prime \prime}}(t)\left(1+N_{\mathbf{m}}(t)\right),
\end{aligned}
$$

as expected from Fermits Golden Rule.

The quantum Boltzmann equation found above, in combination with the expressions for the shift in the energy levels, fully describes the dynamics of the gas in the normal state. However, close to the critical temperature when the occupation numbers of the one-particle groundstate $\chi_{\mathbf{o}}(\mathrm{x})$ start to become large, we are no longer allowed to neglect the nonlinear terms in the Fokker-Planck equation in equation (3.110). Applying again the Hartree-Fock approximation, which is essentially exact in the weak-coupling limit as we have seen in the equilibrium case, and substituting the ansatz $P\left[\phi^{*}, \phi ; t\right]=P_{0}\left[\phi_{\mathbf{0}}^{*}, \phi_{0} ; t\right] P_{1}\left[\phi^{\prime *}, \phi^{\prime} ; t\right]$ into equation $(3.110)$, we obtain for the probability distribution of the condensate

$$
\begin{aligned}
i \hbar \frac{\partial}{\partial t} P_{0}\left[\phi_{\mathbf{0}}^{*}, \phi_{\mathbf{0}} ; t\right]= & -\frac{\partial}{\partial \phi_{\mathbf{0}}}\left(\epsilon_{\mathbf{0}}+\hbar \Sigma_{\mathbf{0}}^{(+)}-\mu(t)\right. \\
& \left.+T_{\mathbf{0}, \mathbf{0} ; \mathbf{0 . 0}}^{(+)}(t)\left|\phi_{\mathbf{0}}\right|^{2}\right) \phi_{\mathbf{0}} P_{\mathbf{0}}\left[\phi_{\mathbf{0}}^{*}, \phi_{\mathbf{0}} ; t\right] \\
& +\frac{\partial}{\partial \phi_{\mathbf{0}}^{*}}\left(\epsilon_{\mathbf{0}}+\hbar \Sigma_{\mathbf{0}}^{(-)}-\mu(t)\right. \\
& \left.+T_{\mathbf{0}, \mathbf{0} ; \mathbf{0}, \mathbf{0}}^{(+)}(t)\left|\phi_{\mathbf{0}}\right|^{2}\right) \phi_{\mathbf{0}}^{*} P_{\mathbf{0}}\left[\phi_{\mathbf{0}}^{*}, \phi_{\mathbf{0}} ; t\right] \\
& -\frac{1}{2} \frac{\partial^{2}}{\partial \phi_{\mathbf{0}}^{*} \partial \phi_{\mathbf{0}}} \hbar \Sigma_{\mathbf{0}}^{K} P_{\mathbf{0}}\left[\phi_{\mathbf{0}}^{*}, \phi_{\mathbf{0}} ; t\right],
\end{aligned}
$$

where it should be remembered that for consistency reasons the selfenergies need to be evaluated at the energies $\epsilon_{\mathbf{0}}^{\prime \prime}(t)=\epsilon_{\mathbf{0}}^{\prime}(t)+T_{\mathbf{0 , 0 ; 0 , 0}}^{(+)}(t)\left|\phi_{\mathbf{0}}\right|^{2}$. In equilibrium the fluctuation-dissipation theorem then reads

$$
i R_{\mathbf{n}}=-\frac{\beta}{4} \hbar \Sigma_{\mathbf{0}}^{\mathrm{K}}\left(\epsilon_{\mathbf{0}}^{\prime}-\mu+T_{\mathbf{0 , 0 ; 0 , 0}}^{(+)}\left|\phi_{\mathbf{0}}\right|^{2}\right) \text {, }
$$


which guarantees that the probability distribution for the condensate relaxes to the correct equilibrium distribution

$$
P_{0}\left[\phi_{\mathbf{0}}^{*}, \phi_{\mathbf{0}}: \infty\right] \propto \exp \left\{-\beta\left(\epsilon_{\mathbf{0}}^{\prime}-\mu+\frac{T_{\mathbf{0 . 0}: \mathbf{0} .0}^{(+)}}{2}\left|\phi_{0}\right|^{2}\right)\left|\phi_{\mathbf{0}}\right|^{2}\right\} .
$$

The Fokker-Planck equation, and hence the Boltzmamn equation, for the noncondensed part of the gas is the same as in the normal state because for a discrete system the contributions from the condenstate to the selfenergies is already included in our expressions for $\hbar \Sigma_{\mathrm{n}}^{( \pm), K}$ and needs not be separated out explicitly. This implies of course, that if we evaluate the rates $\Gamma_{\mathbf{n}}^{\text {ont }}(t)$ and $\Gamma_{\mathbf{n}}^{\mathrm{in}}(t)$ by replacing the sum over states by an integral over a continuum. we first need to separate the contribution from the condensate to obtain the correct result.

In summary we have thus found that in the weak-coupling limit the dynamics of Bose-Einstein condensation in an external trapping potential is described by the coupled equations in equation (3.122) and equation (3.125). i.e., by a Boltzmann equation for the noncondensed part of the gas and a Fokker-Planck equation for the condensate, respectively. If we apply these equations to a Bose gas with a positive scattering length, we essentially arrive at the theory recently put forward by Gardiner et al. [93]. Indeed, neglecting the energy shifts and assuming that the noncondensed cloud is in equilibrium with a chemical potential $\mu>\epsilon_{0}$, our Fokker-Planck equation for a $U(1)$ invariant probability distribution of the condensate becomes

$$
\begin{aligned}
i \hbar \frac{\partial}{\partial t} P_{0}\left[\left|\phi_{\mathbf{0}}\right| ; t\right]= & -\frac{\beta}{4} \hbar \Sigma_{\mathbf{0}}^{\mathrm{K}} \frac{\partial}{\partial \phi_{\mathbf{0}}}\left(\epsilon_{0}-\mu+T_{\mathbf{0}, \mathbf{0} ; \mathbf{0 . 0}}^{(+)}\left|\phi_{\mathbf{0}}\right|^{2}\right) \phi_{\mathbf{0}} P_{0}\left[\left|\phi_{\mathbf{0}}\right| ; t\right] \\
& -\frac{\beta}{4} \hbar \Sigma_{0}^{\mathrm{K}} \frac{\partial}{\partial \phi_{\mathbf{0}}^{*}}\left(\epsilon_{\mathbf{0}}-\mu+T_{\mathbf{0}, \mathbf{0} ; \mathbf{0}, \mathbf{0}}^{(+)}\left|\phi_{\mathbf{0}}\right|^{2}\right) \phi_{\mathbf{0}}^{*} P_{0}\left[\left|\phi_{\mathbf{0}}\right| ; t\right] \\
& -\frac{1}{2} \hbar \Sigma_{\mathbf{0}}^{\mathrm{K}} \frac{\partial^{2}}{\partial \phi_{\mathbf{0}}^{*} \partial \phi_{\mathbf{0}}} P_{0}\left[\left|\phi_{\mathbf{0}}\right| ; t\right] .
\end{aligned}
$$

It is equivalent to the Fokker-Planck equation for a single-mode laser, since introduction of the dimensionless time $\tau=t\left(i \Sigma_{\mathbf{0}}^{\mathrm{K}} / 8\right)\left(2 \beta T_{\mathbf{0} . \mathbf{0} ; \mathbf{0}, \mathbf{0}}^{(+)}\right)^{1 / 2}$, the dimensionless condensate number $I=\left|\phi_{\mathbf{0}}\right|^{2}\left(2 \beta T_{\mathbf{0}, \mathbf{0} ; \mathbf{0 . 0}}^{(+)}\right)^{1 / 2}$, and the so-called "pump parameter" $a=2 \beta\left(\mu-\epsilon_{\mathbf{0}}\right) /\left(2 \beta T_{\mathbf{0}, \mathbf{0} ; \mathbf{0 . 0}}^{(+)}\right)^{1 / 2}$ leads to the FokkerPlanck equation

$$
\frac{\partial}{\partial \tau} P[I ; \tau]=\frac{\partial}{\partial I}\left\{2(I-a) I+4 I \frac{\partial}{\partial I}\right\} P[I ; \tau]
$$

well-known from laser theory [94]. The typical behaviour of $P[I ; \tau]$ during Bose-Einstein condensation in shown in Figure 17. 


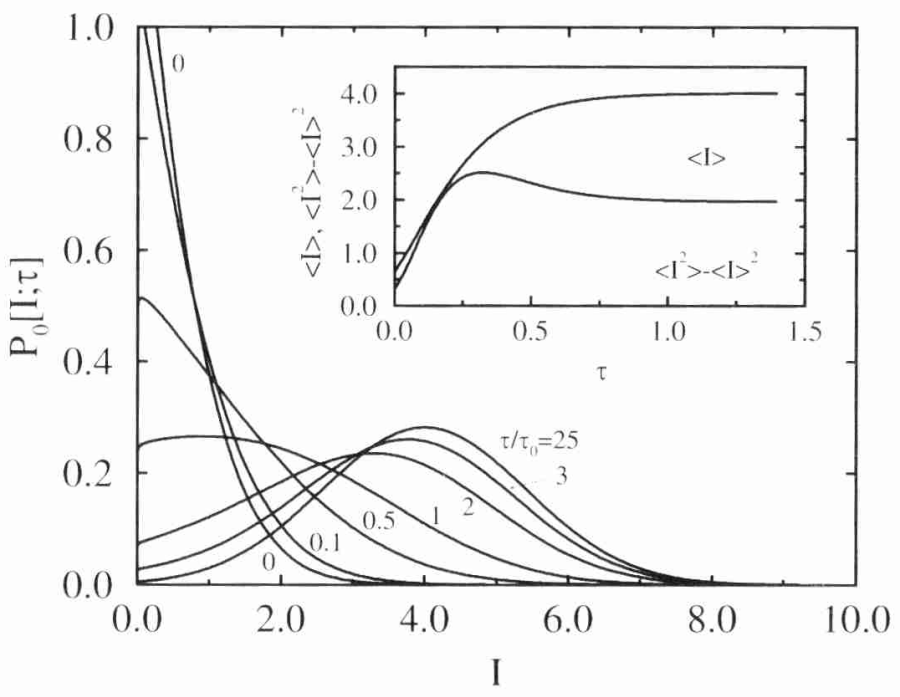

Fig. 17. Typical evolution of the condensate probability distribution during BoseEinstein condensation. The slowest relaxation rate is $1 / \tau_{0} \simeq 5.7$. The inset shows the evolution of the average condensate number and its fluctuations.

Calculating $\langle I\rangle(\tau)$ from equation (3.128) by making use of the meanfield assumption $\left\langle I^{2}\right\rangle(\tau)=(\langle I\rangle(\tau))^{2}$, precisely reproduces the theory of Gardiner et al. A comparison with the experiments of Miesner et al. shows that the theory of Gardiner and coworkers is unfortunately not fully satisfactory $[95,96]$. The reason for this is presumably that these experiments are really in the strong-coupling limit, where the above theory does not apply. As mentioned previously, the theory is however applicable to gases with a negative scattering length. Since we know from our experience with homogeneous gases that the chemical potential will never be larger than $\epsilon_{0}^{\prime}$ in this case, we clearly see from the equilibrium probability distribution $P_{0}\left[\phi_{0}^{*}, \phi_{0} ; \infty\right]$ that a condensate in a gas with effectively attractive interactions is only metastable and will ultimately collapse to a dense state after it has overcome an energy barrier, either by thermal fluctuations or by quantum mechanical tumneling. This is clearly in full agreement with the discussion in Section 3.1. Furthermore, we now also understand how the dynamics of the collapse must be treated. It requires the solution of a stochastic, dissipative nonlinear Schrödinger equation, in which the dissipative terms and the noise obey an appropriate fluctuation-dissipation theorem. Having arrived at this important result, we are finally left with a discussion of the strong-coupling limit. 


\subsubsection{Strong-coupling limit}

The difficulty with the strong-coupling limit is that the appropriate oneparticle states of the gas are no longer the states $\chi_{\mathbf{n}}(\mathbf{x})$ of the trapping potential because these states are now strongly coupled by the mean-field interaction. For our Caldeira-Leggett model this problem could be easily resolved by introducing the states $\chi_{\mathbf{n}}^{\prime}(\mathbf{x})$ that incorporate the average interaction with the reservoir. However, for a trapped Bose gas the same procedure would lead to an expansion into states $\chi_{\mathbf{n}}^{\prime}(\mathrm{x}: t)$ that parametrically depend on time since the mean-field interaction depends on time also. In principle, we thus have to deal with the situation that not only the occupation numbers vary with time but also the states to which these occupation numbers belong. This results in highly nontrivial dynamics since it is a priori clear that an adiabatic approximation. in which we neglect the nondiagonal matrix elements $\int \mathrm{d} \mathrm{x} \chi_{\mathbf{n}}^{\prime *}(\mathrm{x} ; t)(\partial / \partial t) \chi_{\mathbf{n}^{\prime}}^{\prime}(\mathrm{x}: t)$, is not valid, because of the lack of a separation of time scales. Notice that we were able to circumvent the above problems for a Bose gas with effectively attractive interactions due to the fact that on the one hand the growth of the condensate essentially takes place in the weak-coupling regime and that on the other hand the collapse, which certainly occurs in the strong-coupling regime, requires in first instance only knowledge of the collisionless dynamics of the condensate.

Notwithstanding the above romarks, we can make progress in the strong-coupling limit if we realize that in this limit the typical length scale on which the (noncondensate) density of the gas varies is always much larger than the trap size. We are therefore justified in performing a gradient cxpansion. To do so in a systematic fashion, it is convenicnt to express the various selfenergies and interactions in the coordinate representation, i.e., by introducing

$$
h \Sigma^{( \pm), K}\left(\mathbf{x}, \mathbf{x}^{\prime} ; \epsilon\right)=\sum_{\mathbf{n}, \mathbf{n}^{\prime}} \chi_{\mathbf{n}}(\mathbf{x}) \hbar \Sigma_{\mathbf{n}, \mathbf{n}^{\prime}}^{( \pm), K}(\epsilon) \chi_{\mathbf{n}^{\prime}}^{*}\left(\mathbf{x}^{\prime}\right)
$$

and similarly

$$
V^{( \pm), K}\left(\mathbf{x}, \mathbf{x}^{\prime} ; \mathbf{y}, \mathbf{y}^{\prime} ; \epsilon\right)=\sum_{\mathbf{n}, \mathbf{n}^{\prime}} \sum_{\mathbf{m}, \mathbf{m}^{\prime}} \chi_{\mathbf{n}}(\mathbf{x}) \chi_{\mathbf{m}}(\mathbf{y}) V_{\mathbf{n}, \mathbf{m} ; \mathbf{n}^{\prime}, \mathbf{m}^{\prime}}^{( \pm)}(\epsilon) \chi_{\mathbf{n}^{\prime}}^{*}\left(\mathbf{x}^{\prime}\right) \chi_{\mathbf{m}^{\prime}}^{*}\left(\mathbf{y}^{\prime}\right) .
$$


In this coordinate representation, our Fokker-Planck equation acquires in all generality the form

$$
\begin{aligned}
& i \hbar \frac{\partial}{\partial t} P\left[\phi^{*}, \phi ; t\right]=-\int \mathrm{d} \mathbf{x} d \mathrm{x}^{\prime} \frac{\delta}{\delta \phi(\mathrm{x})}\left\{\left(\left(-\frac{\hbar^{2} \nabla^{2}}{2 m}+V^{\mathrm{ex}}(\mathrm{x})\right.\right.\right. \\
& \left.-\mu(t)) \delta\left(\mathrm{x}-\mathrm{x}^{\prime}\right)+\hbar \Sigma^{(+)}\left(\mathrm{x}, \mathrm{x}^{\prime}\right)\right) \phi\left(\mathrm{x}^{\prime}\right) \\
& \left.+\int \mathrm{d} \mathbf{y d} \mathbf{y}^{\prime} V^{(+)}\left(\mathbf{x}, \mathbf{x}^{\prime} ; \mathbf{y}, \mathbf{y}^{\prime}\right) \phi^{*}(\mathbf{y}) \phi\left(\mathbf{y}^{\prime}\right) \phi\left(\mathbf{x}^{\prime}\right)\right\} P\left[\phi^{*}, \phi ; t\right] \\
& +\int \mathrm{dx} d \mathrm{x}^{\prime} \frac{\delta}{\delta \phi^{*}(\mathrm{x})}\left\{\left(\left(-\frac{\hbar^{2} \nabla^{2}}{2 m}+V^{\mathrm{ex}}(\mathrm{x})\right.\right.\right. \\
& \left.-\mu(t)) \delta\left(\mathrm{x}-\mathrm{x}^{\prime}\right)+\hbar \Sigma^{(-)}\left(\mathrm{x}^{\prime}, \mathrm{x}\right)\right) \phi^{*}\left(\mathrm{x}^{\prime}\right) \\
& \left.+\int \mathrm{d} \mathbf{y} d y^{\prime} V^{(-)}\left(\mathbf{x}^{\prime}, \mathbf{x}: \mathbf{y}^{\prime}, \mathbf{y}\right) \phi^{*}\left(\mathbf{x}^{\prime}\right) \phi^{*}\left(\mathbf{y}^{\prime}\right) \phi(\mathbf{y})\right\} P\left[\phi^{*}, \phi ; t\right] \\
& -\frac{1}{2} \int \mathrm{d} \mathrm{x} d \mathrm{x}^{\prime} \frac{\delta^{2}}{\delta \phi(\mathrm{x}) \delta \phi^{*}\left(\mathrm{x}^{\prime}\right)}\left\{\hbar \Sigma^{\mathrm{K}}\left(\mathrm{x}, \mathrm{x}^{\prime}\right)\right. \\
& \left.+\int \mathrm{d} \mathbf{y d} \mathbf{y}^{\prime} V^{\mathrm{K}}\left(\mathbf{x}, \mathbf{x}^{\prime} ; \mathbf{y}, \mathbf{y}^{\prime}\right) \phi^{*}(\mathbf{y}) \phi\left(\mathbf{y}^{\prime}\right)\right\} P\left[\phi^{*}, \phi ; t\right] .
\end{aligned}
$$

To study its physical content and to familiarize ourselves with how to perform the desired gradient expansion, we first analyze again the normal state of the gas in which we can neglect the nonlinear terms representing the effect of the condensate.

In the normal state the condensate is absent and we are only interested in the behavior of the quantity $\left\langle\phi(\mathbf{x}) \phi^{*}\left(\mathbf{x}^{\prime}\right)\right\rangle(t)$. From the above Fokker-Planck equation we see that it obcys the equation of motion

$$
\begin{aligned}
i \hbar \frac{\partial}{\partial t}\left\langle\phi(\mathrm{x}) \phi^{*}\left(\mathrm{x}^{\prime}\right)\right\rangle(t)= & \int \mathrm{d} \mathrm{x}^{\prime \prime}\left(\left(-\frac{\hbar^{2}}{2 m} \frac{\partial^{2}}{\partial \mathrm{x}^{2}}+V^{\mathrm{ex}}(\mathrm{x})-\mu(t)\right)\right. \\
& \left.\times \delta\left(\mathrm{x}-\mathrm{x}^{\prime \prime}\right)+\hbar \Sigma^{(+)}\left(\mathrm{x}, \mathrm{x}^{\prime \prime}\right)\right)\left\langle\phi\left(\mathrm{x}^{\prime \prime}\right) \phi^{*}\left(\mathrm{x}^{\prime}\right)\right\rangle(t) \\
& -\int \mathrm{d} \mathrm{x}^{\prime \prime}\left(\left(-\frac{\hbar^{2}}{2 m} \frac{\partial^{2}}{\partial \mathrm{x}^{\prime 2}}+V^{\mathrm{ex}}\left(\mathrm{x}^{\prime}\right)-\mu(t)\right)\right. \\
& \left.\times \delta\left(\mathrm{x}^{\prime \prime}-\mathrm{x}^{\prime}\right)+\hbar \Sigma^{(-)}\left(\mathrm{x}^{\prime \prime}, \mathrm{x}^{\prime}\right)\right)\left\langle\phi(\mathrm{x}) \phi^{*}\left(\mathrm{x}^{\prime \prime}\right)\right\rangle(t) \\
& -\frac{1}{2} \hbar \Sigma^{\mathrm{K}}\left(\mathrm{x}, \mathrm{x}^{\prime}\right) .
\end{aligned}
$$


Introducing now the Wigner distribution [97] by means of

$$
N(\mathbf{x} \cdot \mathbf{k}: t)+\frac{1}{2}=\int \mathrm{d} \mathbf{x}^{\prime} \mathrm{e}^{-i \mathbf{k} \cdot \mathbf{x}^{\prime}}\left\langle\phi\left(\mathbf{x}+\mathrm{x}^{\prime} / 2\right) \phi^{*}\left(\mathbf{x}-\mathbf{x}^{\prime} / 2\right)\right\rangle(t)
$$

and similarly the selfenergies $\hbar \Sigma^{( \pm)} \cdot \mathrm{K}(\mathrm{x}, \mathrm{k})$ by

$$
h \Sigma^{( \pm) \cdot \mathrm{K}}(\mathrm{x} \cdot \mathrm{k})=\int \mathrm{d} \mathrm{x}^{\prime} \mathrm{c}^{-i \mathrm{k} \cdot \mathrm{x}^{\prime}} h \Sigma^{( \pm) \cdot \mathrm{K}}\left(\mathrm{x}+\mathrm{x}^{\prime} / 2 \cdot \mathrm{x}-\mathrm{x}^{\prime} / 2\right) .
$$

we find after substituting these relations in lowest nonvanishing order in the gradient $\partial / \partial \mathbf{x}$ the expected quantum Boltzmann equation [77.83]

$$
\begin{aligned}
\frac{\partial}{\partial t} N(\mathrm{x}, \mathrm{k} ; t) & +\frac{1}{\hbar} \frac{\partial \epsilon^{\prime}(\mathrm{x}, \mathrm{k}: t)}{\partial \mathbf{k}} \cdot \frac{\partial}{\partial \mathrm{x}} N(\mathrm{x}, \mathrm{k}: t)-\frac{1}{\hbar} \frac{\partial \epsilon^{\prime}(\mathrm{x}, \mathrm{k}: t)}{\partial \mathrm{x}} \cdot \frac{\partial}{\partial \mathrm{k}} N(\mathrm{x}, \mathrm{k}: t) \\
= & -\Gamma^{\text {out }}(\mathrm{x} \cdot \mathrm{k}: t) N(\mathrm{x}, \mathrm{k}: t)+\Gamma^{\mathrm{in}}(\mathrm{x}, \mathrm{k}: t)(1+N(\mathrm{x} \cdot \mathrm{k}: t))
\end{aligned}
$$

where the energies of the atoms have to be determined selfconsistently from

$$
\epsilon^{\prime}(\mathbf{x}, \mathbf{k} ; t)=\epsilon(\mathbf{k})+V^{e x}(\mathbf{x})+\operatorname{Re}\left[\hbar \Sigma^{(+)}\left(\mathbf{x}, \mathbf{k} ; \epsilon^{\prime}(\mathbf{x}, \mathbf{k} ; t)-\mu(t)\right)\right] .
$$

Moreover, the expressions for the retarded energy $\hbar \Sigma^{(+)}\left(\mathbf{x} . \mathbf{k} ; \epsilon^{\prime}(\mathbf{x}, \mathbf{k} ; t)-\right.$ $\mu(t))$, and the collision rates $\Gamma^{\text {ont }}(\mathrm{x}, \mathrm{k} ; t)$ and $\Gamma^{\mathrm{in}}(\mathrm{x}, \mathrm{k} ; t)$ turn out to be identical to the ones for a homogeneous gas $[91,98,99]$. We only need to replace the onc-particle energies $\epsilon^{\prime}(\mathbf{k} ; t)$ and occupation numbers $N(\mathbf{k} ; t)$ by their local counterparts $\epsilon^{\prime}(\mathrm{x}, \mathrm{k} ; t)$ and $N(\mathrm{x}, \mathbf{k} ; t)$, respectively. Notice also that the same substitution has to be made in the Bethe-Salpeter equation for the many-body T matrix, which implies that the effective interaction now implicitly depends both on the position $\mathrm{x}$ and the time $t$.

As an example, let us consider the gas not too close to the critical temperature. Then the effective interaction is well approximated by the two-body T matrix and we simply have

$$
\epsilon^{\prime}(\mathbf{x}, \mathbf{k} ; t)=\epsilon(\mathbf{k})+V^{\mathrm{ex}}(\mathbf{x})+\frac{8 \pi n(\mathbf{x}, t) a \hbar^{2}}{m} .
$$

As a result the equilibrium solution $N(\mathbf{x}, \mathbf{k} ; \infty)$ of the quantum Boltzmann equation equals a Bose-Einstein distribution evaluated at the en$\operatorname{ergy} \epsilon(\mathrm{k})+V^{\operatorname{ex}}(\mathbf{x})+8 \pi n(\mathrm{x}) a \hbar^{2} / m-\mu$. The equilibrium density profile $n(\mathrm{x})=\int \mathrm{d} \mathbf{k} N(\mathbf{x}, \mathbf{k} ; \infty) /(2 \pi)^{3}$ thus corresponds to a local-density approximation but is indeed very accurate for the atomic gases of interest. Apart from describing the relaxation of the gas towards equilibrium, the quantum Boltzmann equation can also be used to study the collective modes of the trapped gas above the critical temperature. Using the same methods that have recently been applied to inhomogeneous Bose gases by Zaremba 
et al. [100], we can for example derive the hydrodynamic equations of motion the gas. Morcover, the collisionless modes are obtained from

$$
\begin{array}{r}
\frac{\partial}{\partial t} N(\mathbf{x}, \mathbf{k} ; t)+\frac{\hbar \mathbf{k}}{m} \cdot \frac{\partial}{\partial \mathbf{x}} N(\mathbf{x}, \mathbf{k} ; t)-\frac{1}{\hbar} \frac{\partial}{\partial \mathbf{x}}\left(V^{\mathrm{ex}}(\mathbf{x})\right. \\
\left.+\frac{8 \pi n(\mathrm{x}, t) a \hbar^{2}}{m}\right) \cdot \frac{\partial}{\partial \mathrm{k}} N(\mathrm{x}, \mathrm{k} ; t)=0
\end{array}
$$

Solving this equation is equivalent to the Generalized Random-Phase Approximation [83]. More important is that the above collisionless Boltzmann equation can be shown to satisfy the Kohn theorem [101] by substituting the shifted equilibrium profile $N\left(\mathbf{x}-\mathbf{x}_{\mathrm{cm}}(t) . \mathbf{k}-(m / \hbar) \mathrm{dx}_{\mathrm{cm}}(t) / \mathrm{d} t\right.$; $\infty)$. For an harmonic external potential the center-of-mass of the gas cloud then precisely follows Newton's law $\mathrm{d}^{2} \mathbf{x}_{\mathrm{cm}}(t) / \mathrm{d} t^{2}=-\nabla V^{\mathrm{ex}}\left(\mathrm{x}_{\mathrm{cm}}(t)\right)$, which shows that the gas has three collisionless modes with frequencies that are equal to the three frequencies of the extermal trapping potential.

The full quantum Boltzmamn equation in equation (3.135) also describes the relaxation of the gas towards equilibrium. It can thus be used to study forced evaporative cooling by adding an appropriate loss term to account for the escaping high-energetic atoms. In agreement with our previous picture we then expect that, if the evaporative cooling is performed slowly, the distribution function $N(\mathbf{x}, \mathbf{k} ; t)$ can be well approximated by a truncated equilibrium distribution with a time-dependent temperature and chemical potential. As a result, we can use the results from the homogeneous gas to show that in the critical regime the gas again becomes unstable towards Bose-Einstein condensation. This is the case when $\epsilon^{\prime}(\mathbf{x}, 0: t)<\mu(t)$ for positions near the center of the trap where the density of the gas is highest. If that happens we are no longer allowed to neglect the nonlinear terms in the Fokker-Planck equation and some corrections are neccesary. Applying again the Hartee-Fock approximation, which is correct sufficiently close to the critical temperature where $k_{B} T \gg 4 \pi n_{0}(\mathrm{x}, t) a \hbar^{2} / m$, we find first of all that the quantum Boltzmann equation for the noncondensed part of the gas has the same form as in equation (3.135). We only have to add the by now familiar mean-field interaction $2 n_{0}(\mathbf{x}, t) T^{(+)}(\mathbf{0}, 0)$ due to the condensate to the renormalized energy $\epsilon^{\prime}(\mathbf{x}, \mathbf{k} ; t)$. Finally, the scattering rates $\Gamma^{\text {out }}(\mathrm{x}, \mathrm{k} ; t)$ and $\Gamma^{\mathrm{in}}(\mathrm{x}, \mathrm{k} ; t)$ have to be adjusted in precisely the same way as in the homogeneous case to account for the macroscopic occupation of the condensate $[98,102,103]$.

In addition, the resulting quantum Boltzmann equation for the noncondensed atoms is coupled to a Fokker-Planck equation for the condensate. 
After a gradient expansion the latter equals

$$
\begin{aligned}
& i \hbar \frac{\partial}{\partial t} P_{0}\left[\phi_{0}^{*} \cdot \phi_{0}: t\right]=-\int \mathrm{d} \mathbf{x} \frac{\delta}{\delta \phi_{0}(\mathrm{x})}\left(-\frac{h^{2} \boldsymbol{\nabla}^{2}}{2 m}+V^{\mathrm{ex}}(\mathrm{x})-\mu(t)\right. \\
& \left.+\hbar \Sigma^{(+)}(\mathbf{x}, \mathbf{0})+T^{(+)}(\mathbf{0} .0)\left|\phi_{\mathbf{0}}(\mathbf{x})\right|^{2}\right) \phi_{\mathbf{0}}(\mathbf{x}) P_{0}\left[\phi_{0}^{*} \cdot \phi_{\mathbf{0}} ; t\right] \\
& +\int \mathrm{dx} \frac{\delta}{\delta \phi_{0}^{*}(\mathrm{x})}\left(-\frac{\hbar^{2} \nabla^{2}}{2 m !}+V^{\mathrm{ex}}(\mathrm{x})-\mu(t)\right. \\
& \left.+\hbar \Sigma^{(-)}(\mathbf{x}, \mathbf{0})+T^{(+)}(\mathbf{0}, 0)\left|\phi_{0}(\mathbf{x})\right|^{2}\right) \phi_{0}^{*}(\mathbf{x}) P_{0}\left[\phi_{0}^{*}, \phi_{0}: t\right] \\
& -\frac{1}{2} \int \mathrm{d} \mathbf{x} \frac{\delta^{2}}{\delta \phi_{0}(\mathrm{x}) \delta \phi_{\mathbf{0}}^{*}(\mathrm{x})} \hbar \Sigma^{\mathrm{K}}(\mathbf{x}, \mathbf{0}) P_{0}\left[\phi_{0}^{*}, \phi_{\mathbf{0}}: t\right] .
\end{aligned}
$$

where $\hbar \Sigma^{( \pm)} \cdot \mathrm{K}(\mathrm{x}, \mathbf{0})$ denotes $\hbar \Sigma^{( \pm)} \cdot \mathrm{K}(\mathrm{x} . \mathrm{k}=\mathbf{0})$. In more detail we have for the real part of the retarded and advanced selfenergies that $S(\mathrm{x}, 0)=$ $\operatorname{Re}\left[h \Sigma^{( \pm)}\left(\mathrm{x}, 0: \epsilon^{\prime \prime}(\mathrm{x}, \mathbf{0} ; t)-\mu(t)\right)\right]$ and $\epsilon^{\prime \prime}(\mathrm{x}, \mathbf{0}: t)$ the local energy of a condensate atom. Moreover. using our picture of the evaporative cooling of the gas, the imaginary parts follow most conveniently from the fluctuationdissipation theorem

$$
\begin{aligned}
i R(\mathbf{x}, \mathbf{0})= & -\frac{\beta(t)}{4} \hbar \Sigma^{\mathrm{K}}(\mathbf{x}, \mathbf{0}) \\
& \times\left(-\frac{\hbar^{2} \boldsymbol{\nabla}^{2}}{2 m}+\epsilon^{\prime}(\mathbf{x}, \mathbf{0} ; t)-\mu(t)+T^{(+)}(\mathbf{0}, 0)\left|\phi_{\mathbf{0}}(\mathbf{x})\right|^{2}\right)
\end{aligned}
$$

where $\hbar \Sigma^{K}(\mathbf{x}, \mathbf{0})=\hbar \Sigma^{( \pm)}, \mathrm{K}\left(\mathbf{x}, \mathbf{0} ; \epsilon^{\prime \prime}(\mathbf{x}, \mathbf{0} ; t)-\mu(t)\right)$ can in a good approximation be considered as independent of $\left|\phi_{\mathbf{0}}(\mathbf{x})\right|$. Near equilibrim the Fokker-Planck equation thus simplifies to

$$
\begin{aligned}
i \hbar \frac{\partial}{\partial t} P_{0}\left[\left|\phi_{0}\right| ; t\right]= & -\frac{\beta}{4} \int \mathrm{dx} \hbar \Sigma^{\mathrm{K}}(\mathbf{x}, \mathbf{0}) \frac{\delta}{\delta \phi_{\mathbf{0}}(\mathbf{x})}\left(-\frac{\hbar^{2} \boldsymbol{\nabla}^{2}}{2 m}+\epsilon^{\prime}(\mathbf{x}, \mathbf{0})\right. \\
& \left.-\mu+T^{(+)}(\mathbf{0}, 0)\left|\phi_{\mathbf{0}}(\mathbf{x})\right|^{2}\right) \phi_{\mathbf{0}}(\mathbf{x}) P_{0}\left[\left|\phi_{\mathbf{0}}\right| ; t\right] \\
& -\frac{\beta}{4} \int \mathrm{dx} \hbar \Sigma^{\mathrm{K}}(\mathbf{x}, \mathbf{0}) \frac{\delta}{\delta \phi_{\mathbf{0}}^{*}(\mathbf{x})}\left(-\frac{\hbar^{2} \boldsymbol{\nabla}^{2}}{2 m}+\epsilon^{\prime}(\mathbf{x}, \mathbf{0})\right. \\
& \left.-\mu+T^{(+)}(\mathbf{0}, 0)\left|\phi_{\mathbf{0}}(\mathbf{x})\right|^{2}\right) \phi_{\mathbf{0}}^{*}(\mathbf{x}) P_{0}\left[\left|\phi_{\mathbf{0}}\right| ; t\right] \\
& -\frac{1}{2} \int \mathrm{dx} \hbar \Sigma^{\mathrm{K}}(\mathbf{x}, \mathbf{0}) \frac{\delta^{2}}{\delta \phi_{\mathbf{0}}(\mathbf{x}) \delta \phi_{\mathbf{0}}^{*}(\mathbf{x})} P_{0}\left[\left|\phi_{\mathbf{0}}\right| ; t\right]
\end{aligned}
$$


and the probability distribution of the condensate relaxes to the expected stationary state

$$
\begin{array}{r}
P_{0}\left[\left|\phi_{0}\right| ; \infty\right] \times \exp \left\{-\beta \int \mathrm{dx} \phi_{\mathbf{0}}^{*}(\mathbf{x})\left(-\frac{\hbar^{2} \boldsymbol{\nabla}^{2}}{2 m}+\epsilon^{\prime}(\mathbf{x}, \mathbf{0})-\mu\right.\right. \\
\left.\left.+\frac{T^{(+)}(\mathbf{0}, 0)}{2}\left|\phi_{\mathbf{0}}(\mathbf{x})\right|^{2}\right) \phi_{\mathbf{0}}(\mathbf{x})\right\} .
\end{array}
$$

From this result we conclude that equations (3.135) and (3.139) give an accurate discription of the dynamics of Bose-Einstein condensation in the strong-coupling regime. Unfortunately, the solution of these coupled equations is not possible analytically and we therefore have to refer to future work for a numerical study and a comparison of this theory with experiment. We here only remark that the semiclassical approximation in this case amounts to the Thomas-Fermi result for the condensate density

$$
n_{\mathbf{0}}(\mathbf{x}: t)=\frac{1}{T^{(+)}(\mathbf{0}, 0)}\left(\mu(t)-\epsilon^{\prime}(\mathbf{x}, \mathbf{0} ; t)\right) \Theta\left(\mu(t)-\epsilon^{\prime}(\mathbf{x}, \mathbf{0} ; t)\right) .
$$

Such an approximation implicitly assumes, however, that the dominant mechanism for the formation of the condensate is by means of a coherent evolution and not by incoherent collisions. This might be expected not to be very accurate on the basis of the homogencous result that the second mechanism only diverges logarithmically with the system size [90], whereas the first diverges linearly [104]. Nevertheless it seems that only a full solution of equations (3.135) and (3.139) can settle this important issue.

\subsection{Collective modes}

Although we have up to now primarily been focussed on the dynamics of Bose-Einstein condensation itself, it is interesting that we have in fact, similarly as for temperatures above the critical temperature, also arrived at an accurate discription of the collective modes of a condensed gas at nonzero temperatures. Since these collective modes are receiving much attention at present, we now briefly want to discuss how such a description emerges from our Fokker-Planck equation. Since most experiments at present are performed in the collisionless limit, we consider only this limit here. It is in principle possible, however, to derive the hydrodynamic equations of motions as well [100]. Let us for simplicitly first assume that the temperature of the gas is sufficiently far below the critical temperature that the manybody $\mathrm{T}$ matrix is well approximated by the two-body $\mathrm{T}$ matrix, but not so low that the Hartree-Fock approximation is no longer valid. In this regime we have that $\hbar \Sigma^{( \pm)}(\mathbf{x}, \mathbf{k})=8 \pi n^{\prime}(\mathbf{x}, t) a \hbar^{2} / m$ and the dynamics of the noncondensed part of the gas is again determined by the collisionless quantum 
Boltzmann equation given in equation (3.138), where the total density now consists of two contributions

$$
n(\mathbf{x} . t)=n_{\mathbf{0}}(\mathbf{x}, t)+n^{\prime}(\mathbf{x} . t) \equiv|\langle\phi(\mathbf{x})\rangle(t)|^{2}+\int \frac{\mathrm{d} \mathbf{k}}{(2 \pi)^{3}} N(\mathbf{x}, \mathbf{k} ; t) .
$$

The dynamics of the condensate follows again from the nonlinear Schrödinger equation

$$
\begin{aligned}
i \hbar \frac{\partial}{\partial t}\langle\phi(\mathrm{x})\rangle(t)= & \left\{-\frac{\hbar^{2} \nabla^{2}}{2 m}+V^{\mathrm{ex}}(\mathrm{x})-\mu\right. \\
& \left.+\frac{4 \pi a \hbar^{2}}{m}\left(2 n^{\prime}(\mathrm{x}, t)+n_{0}(\mathrm{x}, t)\right)\right\}\langle\phi(\mathrm{x})\rangle(t) .
\end{aligned}
$$

which can be rewritten as the continuity equation

$$
\frac{\partial}{\partial t} n_{0}(\mathbf{x}, t)=-\nabla \cdot\left(\mathbf{v}_{\mathrm{s}}(\mathrm{x}, t) n_{0}(\mathrm{x}, t)\right)
$$

together with the Josephson relation for the superfluid velocity

$$
\begin{aligned}
\frac{\partial}{\partial t} \mathbf{v}_{\mathrm{s}}(\mathrm{x}, t)= & -\nabla\left(V^{\mathrm{ex}}(\mathbf{x})-\mu+\frac{4 \pi a \hbar^{2}}{m}\left(2 n^{\prime}(\mathbf{x}, t)\right.\right. \\
& \left.\left.+n_{0}(\mathbf{x}, t)\right)+\frac{1}{2} m \mathbf{v}_{\mathrm{s}}^{2}(\mathrm{x}, t)\right) .
\end{aligned}
$$

Here, we used that $\langle\phi(\mathrm{x})\rangle(t)=\sqrt{n_{\mathbf{0}}(\mathrm{x}, t)} \mathrm{e}^{i \theta(\mathrm{x}, t)}, \mathbf{v}_{\mathrm{s}}(\mathrm{x}, t)=\hbar \nabla \theta(\mathrm{x}, t) / m$, and have applied the Thomas-Fermi approximation, which is appropriate in the strong-coupling limit. It will presumably not come as a surprise that these coupled equations for $N(\mathbf{x}, \mathbf{k} ; t), n_{\mathbf{0}}(\mathbf{x}, t)$, and $\mathbf{v}_{\mathrm{s}}(\mathbf{x}, t)$ can be shown to fulfill the Kohn theorem exactly. This is an important constraint on any theory for the collective modes of a trapped gas, as stressed recently by Zaremba et al. [100].

As a first step towards this goal we here consider the time-dependent Hartree Fock theory for the collective modes of the Bose condensed gas, in which the collisionless Boltzmann equation in equation (3.138) is coupled to the nonlinear Schrödinger equation in equation (3.145). It turns out that a numerical determination of the eigenmodes of the collisionless Boltzmann equation is rather difficult. Therefore, we have recently proposed a variational approach to solve these coupled equations [105]. Denoting the anisotropic harmonic trapping potential used in the experiments [26,27] by $V^{\operatorname{ex}}(\mathbf{x})=\sum_{j} m \omega_{j}^{2} x_{j}^{2} / 2$, we first of all take for the condensate wave function 
the gaussian ansatz

$$
\begin{aligned}
\langle\phi(\mathbf{x})\rangle(t)= & \sqrt{N_{0}} \prod_{j}\left(\frac{1}{\pi q_{j}^{2}(t)}\right)^{1 / 4} \\
& \times \exp \left\{-\frac{x_{j}^{2}}{2 q_{j}^{2}(t)}\left(1-i \frac{m q_{j}(t)}{\hbar} \frac{\mathrm{d} q_{j}(t)}{\mathrm{d} t}\right)\right\},
\end{aligned}
$$

with the functions $q_{j}(t)$ describing the "breathing" of the condensate in three independent directions. Clearly, this is the anisotropic generalization of the gaussian ansatz that we used in Section 3.1 to study the stability of a condensate with negative scattering length. Although it is not immediately obvious that such an ansatz is also appropriate in the strong-coupling regime of interest here, it has been shown by Perez-Garcia et al. [51] that at zero temperature the assumption of a gaussian density profile nevertheless leads to rather accurate results for the frequencies of the collective modes. We therefore anticipate that at nonzero temperatures the same will be the case.

Next we also need an ansatz for the distribution function $N(\mathrm{x}, \mathrm{k}: t)$ of the thermal cloud. Here, we consider the Bose distribution

$$
\begin{aligned}
N(\mathrm{x}, \mathrm{k}: t)= & N^{\prime} \frac{1}{\zeta(3)}\left(\prod_{j} \frac{\hbar \omega_{j}}{k_{B} T\left\langle\alpha_{j}\right\rangle^{2}}\right) \\
& \times\left(\operatorname { e x p } \left\{\sum _ { j } \frac { 1 } { k _ { B } T \langle \alpha _ { j } \rangle ^ { 2 } } \left(\frac{\hbar^{2} \alpha_{j}^{2}(t)}{2 m}\left(k_{j}-\frac{m x_{j}}{\hbar \alpha_{j}(t)} \frac{\mathrm{d} \alpha_{j}(t)}{\mathrm{d} t}\right)^{2}\right.\right.\right. \\
& \left.\left.\left.+\frac{m \omega_{j}^{2}}{2} \frac{x_{j}^{2}}{\alpha_{j}^{2}(t)}\right)\right\}-1\right)^{-1},
\end{aligned}
$$

where $\alpha_{j}(t)$ are again three scaling parameters, $N^{\prime}=N-N_{0}$ is the number of noncondensed particles, $\left\langle\alpha_{j}\right\rangle$ denotes the equilibrium value of $\alpha_{j}(t)$ that in general is greater than 1 due to the repulsive interactions between the atoms, and $\zeta(3) \simeq 1.202$. The functional form of the distribution function is physically motivated by the following reasons. First, by integrating over the momentum $\hbar \mathbf{k}$ we see that the time-dependent density profile of the noncondensed cloud $n^{\prime}(\mathbf{x}, t)$ is a time-independent function of $x_{j} / \alpha_{j}(t)$, as desired. Second, for such a density profile we must have that the local velocity $\hbar\left\langle k_{j}\right\rangle(\mathbf{x}, t) / m$ is given by $\left(x_{j} / \alpha_{j}(t)\right) \mathrm{d} \alpha_{j}(t) / \mathrm{d} t$. Third, we want the equilibrium distribution to be only anisotropic in coordinate space, and not in momentum space. This explains the appearance of the factor $1 /\left\langle\alpha_{j}\right\rangle^{2}$ in the exponent.

Using the ideal gas result for the number of condensate particles and substituting the above ansatz for the condensate wave function and the 


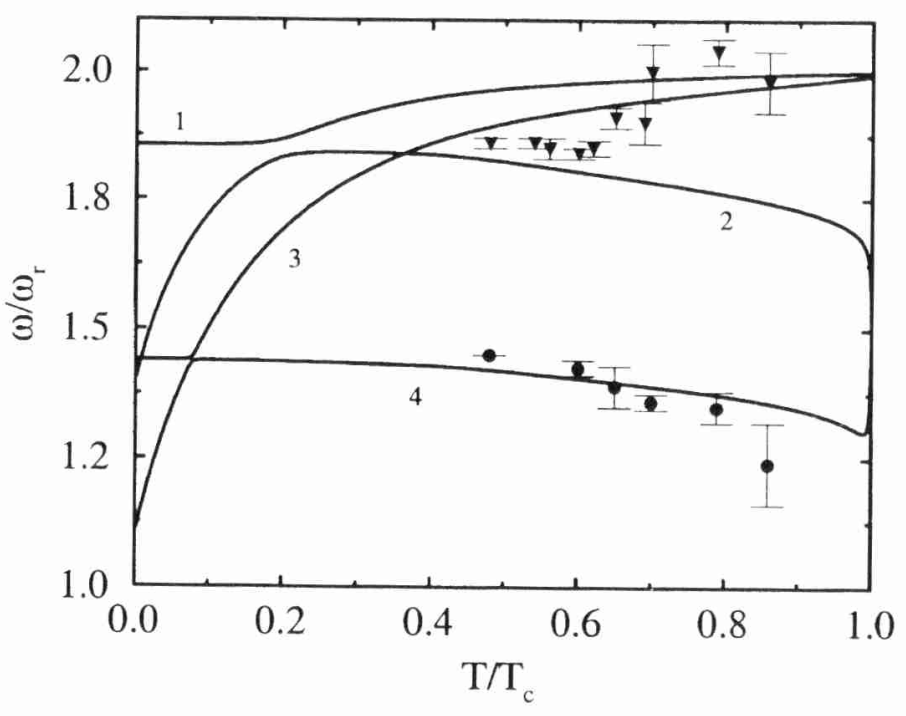

Fig. 18. Collsionless modes in a Bose condensed ${ }^{8} 7 \mathrm{Rb}$ gas. The full lines give the results with a Bose distribution ansatz for the distribution function of the thermal clond. Curves 1 and 2 correspond to the $m=0$ in and out-of-phase modes, respectively. Similarly, curves 3 and 4 give the frequencies of the $m=2$ in and out-of-phase modes. The experimental data is also shown. Triangles are for a $m=0$ mode and circles for a $m=2$ mode.

Wigner distribution function into the nonlinear Schrödinger and collisionless Boltzmamn equations, it is straightforward but somewhat tedious to derive the six coupled equations of motion for the "breathing" parameters $q_{j}(t)$ and $\alpha_{j}(t)$. Linearizing these equations around the equilibrium solutions $\left\langle q_{j}\right\rangle$ and $\left\langle\alpha_{j}\right\rangle$, respectively, we can then also obtain the desired eigenmodes and eigenfrequencies. The results of this calculation for the trap parameters of Jin et al. [26] are shown in Figure 18 together with the experimental data. Note that the trap has a disk geometry with $\omega_{x}=\omega_{y} \equiv \omega_{\mathrm{r}}$ and $\omega_{z}=\sqrt{8} \omega_{\mathrm{r}}$. Due to the axial symmetry, the modes of the gas can be labeled by the usual quantum number $m$ that describes the angular dependence around the $z$-axis. On the whole there appears to be a quite reasonable agreement between this simple theory and experiment. The only exceptions are the two data point almost halfway in between the in and out-of-phase $m=0$ modes. A possible explanation for this discrepancy is that experimentally both modes are excited simultaneously [106]. To make sure of this, however, we need to determine the oscillator strength of the various modes and it appears that the in-phase mode indeed dominates the response for temperatures larger than about $0.6 T_{\mathrm{C}}[105]$. 


\section{Outlook}

With the latter remark we end this course on the use of field-theoretical methods for the study of the equilibrium and nonequilibrium properties of trapped atomic gases. Although we have in principle illustrated all the necessary tools for an ab initio treatment of these new quantum systems. many interesting topics still need to be considered in detail. We already mentioned such topics as Fermi-Bose mixtures, the dynamics of condensate collapse, the strong-coupling theory for the formation of a condensate in either atomic Bose of Fermi gases, and the hydrodynamics of single or multicomponent atomic gases. Several other important areas of research, which can also be easily addressed within the context of quantum field theory, are atom lasers, the damping of collective modes, the behaviour of spinor condensates, the dynamics of topological excitations such as kinks, vortices and skyrmions, quantum critical phenomena, the optical properties of superfluid gases, and two-dimensional phase transitions. In view of these and many other possibilities that are now experimentally feasible, it appears certain that the physics of degenerate atomic gases will remain very exciting for years to come.

\section{References}

[1] E. Abers and B.W. Lee, Phys. Rep. 9C (1973) 1.

[2] K.G. Wilson and J. Kogut, Phys. Rep. 12 (1974) 75.

[3] P.C. Hohenberg and B.I. Halperin, Rev. Mod. Phys. 49 (1977) 435.

[4] S. Sachdev, in Proceedings of the 19th IUPAP International Conference on Statistical Physics, edited by B.-L. Hao (World Scientific, Singapore, 1996).

[5] P.A. Lee and T.V. Ramakrishnan, Ren. Mod. Phys. 57 (1985) 287.

[6] P.W. Anderson, Phys. Rev. 109 (1958) 1492.

[7] R.B. Laughlin, in The Quantum Hall Effect, edited by R.E. Prange and S.M. Girvin (Springer-Verlag, New York, 1990).

[8] X.G. Wen, Phys. Rev. B 43 (1991) 11025.

[9] M.L. Mehta, Random Matrices (Academic Press, New York, 1991).

[10] M.H. Anderson, J.R. Ensher, M.R. Matthews, C.E. Wieman and E.A. Cornell, Sci 269 (1995) 198.

[11] C.C. Bradley, C.A. Sackett, J.J. Tollett and R.G. Hulet, Phys. Rev, Lett. 75 (1995) 1687; C.C. Bradley, C.A. Sackett and R.G. Hulet, ibid. 78 (1997) 985.

[12] K.B. Davis, M.-O. Mewes, M.R. Andrews, N.J. van Druten, D.S. Durfee, D.M. Kurn and W. Ketterle, Phys. Rev. Lett. 75 (1995) 3969.

[13] A. Einstein, Sitz. Kgl. Preuss. Akad. Wiss. (Berlin) (1925) 3.

[14] I.F. Silvera and J.T.M. Walraven, Phys. Rev. Lett. 44 (1980) 164.

[15] A.L. Fetter and J.D. Walecka, Quantum Theory of Many-Particle Systems (McGraw-Hill, New York, 1971). Note that these authors use the more common notation $\hat{a}_{\mathbf{n}, \alpha}$ and $\hat{a}_{\mathbf{n}, \alpha}^{\dagger}$ for the bosonic creation and annihilation operators.

[16] L. Mandel and E. Wolf, Optical Coherence and Quantum Optics (Cambridge, New York, 1995). 
[17] J.W. Negele and H. Orland. Quantum Many-Particle Systems (Addison-Wesley, New York, 1988).

[18] D.J. Amit, Field Theory, the Renormalization Group, and Critical Phenomena (World Scientific. Singapore, 1984).

[19] J. Zinn-Justin. Quantum Field Theory and Critical Phenomena (Oxford. New York. 1989).

[20] F.J. Dyson, Phys. Rev. 75 (1949) 1736.

[21] R.L. Stratonovich, Sov. Phys. Dok: 2 (1958) 416.

[22] J. Hubbard, Phys. Rev. Lett. 3 (1959) 77.

[23] D.G. Fried, T.C. Killian. L. Willmann, D. Landhuis, S.C. Moss. D. Kleppner and T.J. Greytak, Phys. Rev. Lett. 81 (1998) 3811.

[24] N.N. Bogoliubov. J. Phy.s. (U.S.S.R.) 11 (1947) 23.

[25] L.P. Pitaevskii. Sov. Phys. JETP 13 (1961) 451 and E.P. Gross, J. Math. Phys. 4 (1963) 195.

[26] D.S. Jin, J.R. Ensher, M.R. Matthews, C.E. Wieman and E.A. Comell, Phys. Rev. Lett. 77 (1996) 420; D.S. Jin, M.R. Matthews, J.R. Ensher. C.F. Wieman and E.A. Cornell, ibid. 78 (1997) 764 .

[27] M.-O. Mewes, M.R. Anderson, N.J. van Druten, D.M. Kurn. D.S. Durfee, C.G. Townsend and W. Ketterle, Phys. Rev. Lett. 77 (1996) 988.

[28] L.V. Hau, B.D. Busch, C. Liu, Z. Dutton, M.M. Burns and J.A. Golovchenko, Phys. Rev. A 58 (1998) R54.

[29] K.G. Singh and D.S. Rokhsar. Phys. Rev. Lett. 77 (1996) 1667.

[30] M. Edwards, P.A. Ruprecht, K. Burnett, R.J. Dodd and C.W. Clark, Phys. Rev. Lett. 77 (1996) 1671.

[31] A.L. Fetter, Ann. Phys. 7J i:972; 67.

[32] M. Lewenstein and L. You, Plys. Rev. Lett. 77 (1996) 3489

[33] V.N. Popov, Functional Integrals in Quantum Field Theory and Statistical Physics (Reidel, Dordrecht, 1983) and references therein.

[34] F. Dalfovo, S. Giorgini, L. Pitaevskii and S. Stringari, Rev. Mod. Phys. 71 (1999) 463.

[35] D.A.W. Hutchinson, E. Zaremba and A. Griffin, Phys. Rev. Lett. 78 (1997) 1842.

[36] R.J. Dodd, M. Edwards, C.W. Clark and K. Burnett, Phys. Rev. A 57 (1998) R32.

[37] B.A. Lippmann and J. Schwinger, Phys. Rev. 79 (1950) 469.

[38] M. Bijlsma and H.T.C. Stoof, Phys. Rev. A 54 (1996) 5085.

[39] H. Shi and A. Griffin, Phys. Rep. 304 (1998) 1.

[40] J. Bardeen, L.N. Cooper and J.R. Schrieffer, Phys. Rev. 108 (1957) 1175.

[41] H. Kleinert, Forts. Phys. 26 (1978) 565.

[42] E.R.I. Abraham, W.I. McAlexander, J.M. Gerton, R.G. Hulet, R. Côté and A. Dalgarno, Phys. Rev. A 55 (1997) R3299.

[43] H.T.C. Stoof, M. Houbiers, C.A. Sackett, and R.G. Hulet, Phys, Rev. Lett. 76 (1996) 10; M. Houbiers, R. Ferwerda, H.T.C. Stoof, W.1. McAlexander, C.A. Sackett and R.G. Hulet, Phys. Rev. A 56 (1997) 4864.

[44] For an equilibrium argument at zero temperature see L.D. Landau and E.M. Lifshitz, Statistical Physics (Pergamon, London, 1958) and P. Nozières, in BoseEinstein Condensation, edited by A. Griffin, D.W. Snoke and S. Stringari (Cambridge, New York, 1995) p. 15. Nonzero temperatures are discussed in H.T.C. Stoof, Phys. Rev. A 49 (1995) 4704.

[45] M. Houbiers and H.T.C. Stoof, Phys. Rev. A 54 (1996) 5055.

[46] T. Bergeman, Phys. Rev. A 55 (1997) 3658. 
[47] See, for instance, S. Coleman in Aspects of Symmetry (Cambridge, New York. $1985)$.

[48] J.A. Freire and D.P. Arovas, Phys. Rev. A 59 (1999) 1461.

[49] S. Stringari. Phys. Rev. Lett. 77 (1996) 2360.

[50] Y. Castin and R. Dum, Phys. Rev. Lett. 77 (1996) 5315.

[51] V.M. Perez-Garcia, H. Michinel, J.I. Cirac, M. Lewenstein and P. Zoller, Phys. Rev. Lett. 77 (1996) 5320.

[52] R... Dodd, M. Fdwards, C.J. Williams, C.W. Clark, M..J. Holland. P.A. Ruprecht and K. Burnett, Phys. Rev. A 54 (1996) 661.

[53] J. Javanainen, Phys. Rev. A 54 (1996) 3722.

[54] L. You, W. Hoston and M. Lewenstein. Phys. Rev. A 55 (1997) 1581.

[55] P. Öhberg. E.L. Surkov, I. Tittonen, S. Stenholm, M. Wilkens and G.V. Shlyapnikov, Phys, Rev. A 56 (1997) R3346.

[56] For the anisotropic generalization see, for instance, G. Baym and C..J. Pethick. Phys. Rev. Lett. 76 (1996) 6.

[57] A.L. Fetter, Phys. Rev. A 53 (1996) 4245. The exact result is obtained in P.A. Ruprecht, M..J. Holland, K. Burnett and M. Edwards, Phys. Rev. A 51 (1995) 1704.

[58] II.T.C. Stoof, J. Stat. Phys. 87 (1997) 1353. For a different calculation of the tumneling rate that however neglects the phase fluctuations of the condensate. see E.V. Shuryak, Phys. Rev. A 54 (1996) 3151.

[59] C.A. Sackett, C.C. Bradley, M. Welling and R.G. Hulet, Appl. Phys. B 65 (1997) 433.

[60] C.A. Sackett, H.T.C. Stoof and R.G. Hulet, Phys. Rev. Lett. 80 (1998) 2031.

[61] L.P. Pitaevskii, Phys. Lett. A 221 (1996) 14.

[62] C.A. Sackett, J.M. Gerton, M. Welling and R.G. Hulet, Phys. Rev. Lett. 82 (1999) 876.

[63] P.W. Anderson, Phys. Rev. 112 (1958) 1900.

[64] E. Abrahams and T. Tsumeto, Phys. Rev. 152 (1966) 416.

[65] In principle the effective action for a neutral superconductor contains also a topological term, but this does not affect the final result of the following argument because it only leads to a constant shift in the total number of particles. For more details, see M. Stone, Int. J. Mod. Phys. B 9 (1995) 1359 and references therein.

[66] The magnitude of the frequency gap is actually not accurately described by this time-dependent Ginzburg-Landau theory, because the action $S\left[\Delta^{*}, \Delta\right]$ is implicitly only valid for long wavelengths $\hbar v_{\mathrm{F}} k \ll\left|\Delta_{0}\right|$ and low frequencies $\hbar \omega \ll\left|\Delta_{0}\right|$.

[67] This may be compared directly with the results of E.M. Wright, D.F. Walls and J.C. Garrison, Phys. Rev. Lett. 77 (1996) 2158 and K. Molmer, Phys. Rev. A 58 (1998) 566.

[68] V.V. Goldman, I.F. Silvera and A.J. Leggett, Phys. Rev. B 24 (1981) 2870.

[69] For simplicity we here again neglect a topological term in the action, which essentially plays no role in the following. This is also in agreement with our discussion of the superconductor, where exactly the same topological term was not included in the effective action for the global phase [65].

[70] L.H. Thomas, Proc. Camb. Phil. Soc. 23 (1927) 542 and E. Fermi, Mat. Nat. 6 (1927) 602.

[71] See, however, T.-L. Ho and V.B. Shenoy, Phys. Rev. Lett. 77 (1996) 2595.

[72] T.-L. Ho, Phys. Rev. Lett. 81 (1998) 742.

[73] M. Houbiers and H.T.C. Stoof, Phys. Rev. A 59 (1999) 1556.

[74] See, for example, K. Huang, Statistical Mechanics (Wiley, New York, 1987). 
[75] J. Schwinger, J. Math. Phys. 2 (1961) 407.

[76] L.V. Keldysh, Zh. Eksp. Teor. Fiz, 47 (1964) 1515 [Sov. Phys.-JETP 20 (1965) 1018].

[77] P. Danielewicz, Ann. Phys. (N.Y.) 152 (1984) 239.

[78] B. de Wit (private communication).

[79] P. Carruthers and K.S. Dy, Phys. Rev. 147 (1966) 214.

[80] H. Kleinert, Path Integrals in Quantum Mechanies Statistics and Polymer Physics (World Scientific, Singapore, 1994).

[81] This follows from the requirement that we want to be able to Fourier transform the Heaviside function.

[82] A.O. Caldeira and A.J. Leggett, Phys. Rev. Lett. 46 (1981) 211 and A.O. Caldeira and A.J. Leggett, Ann. Phys. (N.Y.) 149 (1983) 374; ibid. 153. (1984) 445.

[83] L.P. Kadanoff and G. Baym, Quantum Statistical Mechanics: Green's Function Methods in Equilibrium and Nonequilibrium Problems (Addison-Wesley. New York. 1962).

[84] D.C. Langreth and J.W. Wilkins, Phys. Rev. B 6 (1972) 3189.

[85] In agreement with our previous remarks, the notation $\int_{t_{0}}$ implies that the time integration can be either from $t_{0}$ to $\infty$ or from $t_{0}$ to $t$.

[86] N.G. van Kampen, Stochastic Processes in Physics and Chemistry (North-Holland. Amsterdam, 1981).

[87] D. Forster. Hydrodynamic Fluctuations, Broken Symmetry, and Correlation Functions (Benjamin, Reading, 1975).

[88] To make a connection with a large body of knowledge in quantum optics, we note that equation (3.104) explicitly shows that the probability distribution $P\left[\phi^{*}, \phi: \ell\right]$ does not correspond to a $P$ or $Q$ representation of the density matrix, but to a Wigner representation instead. The "diffusion matrix" in the Fokker-Planck equation for $P\left[\phi^{*}, \phi ; t\right]$ is therefore diagonal and positive, as is explained by C.W. Gardiner in Quantum Noise (Springer, Berlin, 1991). Chapter 6. The same nice feature will also appear in the case of an interacting Bose gas.

[89] M.A. Kastner, Rev. Mod. Phys. 64 (1992) 849 and references therein.

[90] See also H.T.C. Stoof in Bose-Einstein Condensation, edited by A. Griffin. D.W. Snoke and S. Stringari (Cambridge, New York, 1995) p. 226.

[91] O.J. Luiten, M.W. Reynolds and J.T.M. Walraven, Phys. Rev. A 53 (1996) 381.

[92] N.P. Proukakis and K. Burnett, J. Res. Natl. Inst. Stand. Technol. 101 (1996) 457; N.P. Proukakis, K. Burnett and H.T.C. Stoof, Phys. Rev. A 57 (1998) 1230.

[93] C.W. Gardiner, P. Zoller, R.J. Ballagh and M..J. Davis, Phys. Rev. Lett. 79 (1997) 1793.

[94] H. Risken, Z. Phys. 186 (1965) 85; ibid. 191 (1966) 302.

[95] H.-J. Miesner, D.M. Stamper-Kurn, M.R. Andrews, D.S. Durfee, S. Inouye and W. Ketterle, Sci. 279 (1998) 1005.

[96] For a recent extension of their theory see C.W. Gardiner, M.D. Lee, R.J. Ballagh, M..J. Davis and P. Zoller, Phys. Rev. Lett. 81 (1998) 5266.

[97] M. Hillery, R.F. O'Connell, M.O. Scully and E.P. Wigner, Phys. Rep. 106 (1984) 121.

[98] D.V. Semikoz and I.I. Tkachev, Phys. Rev. Lett. 74 (1995) 3093.

[99] H.T.C. Stoof, J. Low Temp. Phys. 114 (1999) 11.

[100] E. Zaremba, A. Griffin and T. Nikuni, Phys. Rev. A 57 (1998) 4695. See also A. Griffin, W.-C. Wu and S. Stringari, Phys. Rev. Lett. 78 (1997) 1838; G.M. Kavoulakis, C.J. Pethick and H. Smith, Phys. Rev. A 57 (1998) 2938; T. Nikuni and A. Griffin, J. Low. Temp. Phys. 111 (1998) 793, and V. Shenoy and T.-L. Ho, Phys. Rev. Lett. 80 (1998) 3895. 
[101] W. Kohn, Phys. Rev. 123 (1961) 1242.

[102] T.R. Kirkpatrick and J.R. Dorfman, J. Low Temp. Phys. 58 (1985) 301; ibid. 58 (1985) 399

[103] E. Zaremba, T. Nikuni and A. Griffin, J. Low Temp. Phys. 116 (1999) 277.

[104] K. Damle, S.N. Majumdar and S. Sachdev, Phys. Rev. A 54 (1996) 5037.

[105] M.J. Bijlsma and H.T.C. Stoof, Phys. Rev. A 60 (1999).

[106] E.A. Cornell (private communication). 